University of Louisville

ThinkIR: The University of Louisville's Institutional Repository

Electronic Theses and Dissertations

8-2015

\title{
What we see changes how we see: analyzing the plasticity of the horizontal effect.
}

April Marie Schweinhart

University of Louisville

Follow this and additional works at: https://ir.library.louisville.edu/etd

Part of the Experimental Analysis of Behavior Commons

\section{Recommended Citation}

Schweinhart, April Marie, "What we see changes how we see : analyzing the plasticity of the horizontal effect." (2015). Electronic Theses and Dissertations. Paper 2246.

https://doi.org/10.18297/etd/2246

This Doctoral Dissertation is brought to you for free and open access by ThinkIR: The University of Louisville's Institutional Repository. It has been accepted for inclusion in Electronic Theses and Dissertations by an authorized administrator of ThinkIR: The University of Louisville's Institutional Repository. This title appears here courtesy of the author, who has retained all other copyrights. For more information, please contact thinkir@louisville.edu. 
WHAT WE SEE CHANGES HOW WE SEE: ANALYZING THE PLASTICITY OF THE HORIZONTAL EFFECT

\author{
By \\ April Marie Schweinhart \\ B.A., Hanover College, 2008 \\ M.A., University of Louisville, 2012 \\ A Dissertation \\ Submitted to the Faculty of the \\ College of Arts and Sciences of the University of Louisville \\ in Partial Fulfillment of the Requirements \\ for the Degree of \\ Doctor of Philosophy \\ in Experimental Psychology \\ Department of Psychological and Brain Sciences \\ University of Louisville \\ Louisville, Kentucky,
}

August 2015 

WHAT WE SEE CHANGES HOW WE SEE:

ANALYZING THE PLASTICITY OF THE HORIZONTAL EFFECT

\author{
By
}

April Marie Schweinhart

B.A., Hanover College, 2008

M.A., University of Louisville, 2012

A Dissertation Approved on

June 18, 2015

by the following Dissertation Committee

\begin{tabular}{c}
\hline Dissertation Director \\
Edward A. Essock, PhD \\
\\
\hline
\end{tabular}

Patrick Shafto, PhD

Bruce C. Hansen, PhD

Zijiang He, PhD

Keith Lyle, PhD 


\section{ACKNOWLEDGEMENTS}

I would like to thank my mentoring professor, Dr. Edward Essock for his continual guidance, encouragement, and patience. I would also like to thank all of the committee members, but especially Dr. Bruce Hansen and Dr. Patrick Shafto for their early and continued support of this project. Also, many thanks to my parents, Vicky and Donald Schweinhart for providing me access to exceptional educational opportunities and to my fiancé, the honorable J. Anthony Cash for his unyielding encouragement. Lastly, I would like to thank Dr. Stephen Engel who provided the initial inspiration for this work as well as the preliminary program for video manipulation. 


\title{
ABSTRACT \\ WHAT WE SEE CHANGES HOW WE SEE: ANALYZING THE PLASTICITY OF THE HORIZONTAL EFFECT
}

\author{
April M. Schweinhart
}

June 18, 2015

The relationship between the processing of orientations by the human visual system has been related to the orientation content of the natural environment; horizontal orientations, while predominant in natural environments, are perceived less well than vertical and oblique orientations are perceived best, though they are least prevalent in the natural world. This 'horizontal effect' has further extended the well-studied relationship between visual encoding and natural scene statistics as the differential perception of orientations in broadband scenes inversely matches their differential representation in the natural environment. However, the original hypothesis that this relationship may have evolved across millennia in order to make the visual system an efficient informationtransmitting system has been called into question by research showing the modification of orientation perception by exposure to altered environments and studies showing a later development of adult-like orientation processing. Recent work into the effects of adaptation on visual encoding of the natural environment have led me to the conclusion that the relationship between the statistics of the natural world and visual encoding is, in a way, much simpler than previously posited; rather than being adapted over millennia to whiten the typical natural scene anisotropy, the visual system adjusts processing 
dynamically to match the current visual environment. The project presented here details how the statistics of the recently viewed environment affect the way that the visual brain processes information. To assess the effect of recent exposure on broadband orientation processing, the orientation content subjects viewed was modified via fast Fourier transform (FFT) filtering of their environment in near-real-time. Results show that experience in an altered environment modifies anisotropic processing: observers' orientation perception changes from matching the typical environmental distribution to matching that of the recently experienced atypical environment. The results of these experiments can be predicted by assuming that observers' biases of perception are probabilistic and rely on an internal model that matches the recently experienced environmental distribution. This change in perception indicates not only that orientation processing is plastic, but that it is related in a predictable way to an observer's recent visual environment. 


\section{TABLE OF CONTENTS}

PAGE

ACKNOWLEDGEMENTS

ABSTRACT

LIST OF FIGURES

iii

iv

viii

INTRODUCTION 1

Orientation Processing $\quad 3$

Oblique Effect 3

$\begin{array}{ll}\text { Horizontal Effect } & 10\end{array}$

$\begin{array}{ll}\text { Natural Scenes } & 17\end{array}$

$\begin{array}{ll}\text { Orientation Content } & 17\end{array}$

Efficiency and Bayesian Encoding $\quad 19$

$\begin{array}{ll}\text { Adaptation } & 26\end{array}$

Explanation of Perceptual Effects $\quad 26$

Key Sensory Changes $\quad 30$

Recent Relevant Findings $\quad 32$

Perceptual Learning $\quad 35$

Background of the Current Project $\quad 37$

$\begin{array}{ll}\text { GOALS } & 44\end{array}$

$\begin{array}{ll}\text { METHODS } & 46\end{array}$

Testing of Performance at Different Orientations 46

Stimulus Creation and Procedure $\quad 46$

General Adaptation Paradigms and Analyses $\quad 50$

Video Analysis $\quad 52$

EXPERIMENT I $\quad 54$ 
Buildup and Decay of Adaptation $\quad 54$

$\begin{array}{ll}\text { Methods } & 54\end{array}$

$\begin{array}{ll}\text { Results } & 56\end{array}$

$\begin{array}{ll}\text { EXPERIMENT } 2 & 60\end{array}$

Magnitude of Physical Change and Adaptation Effects 60

$\begin{array}{ll}\text { Methods } & 60\end{array}$

$\begin{array}{ll}\text { Results } & 61\end{array}$

$\begin{array}{ll}\text { EXPERIMENT } 3 & 66\end{array}$

Immersive versus Passive Viewing $\quad 66$

$\begin{array}{ll}\text { Methods } & 66\end{array}$

$\begin{array}{ll}\text { Results } & 67\end{array}$

$\begin{array}{ll}\text { EXPERIMENT } 4 & 69\end{array}$

Examining the Effects of an Isotropic Environment 69

$\begin{array}{ll}\text { Methods } & 69\end{array}$

$\begin{array}{ll}\text { Results } & 72\end{array}$

$\begin{array}{ll}\text { CONTROL TESTS } & 79\end{array}$

$\begin{array}{ll}\text { Methods } & 79\end{array}$

$\begin{array}{ll}\text { Results } & 81\end{array}$

GENERAL DISCUSSION AND SUMMARY 86

FOLLOW UP TESTS

$\begin{array}{ll}\text { Methods } & 91\end{array}$

$\begin{array}{ll}\text { Results } & 92\end{array}$

$\begin{array}{ll}\text { MODELING } & 94\end{array}$

$\begin{array}{ll}\text { Results } & 95\end{array}$

$\begin{array}{ll}\text { CONCLUSIONS } & 103\end{array}$

REFERENCES 121

IRB APPROVAL LETTER 144

CURRICULUM VITA 146 


\section{LIST OF FIGURES}

FIGURE

PAGE

1. Creation of incremented, spatially broadband, oriented noise stimuli.

2. Example stimuli used in the matching task $\quad 47$

3. Example of a subject undergoing adaptation. 51

4. Steps in the filtering process $\quad 55$

5. Summarized results of Experiment 1

6. The average time to return to baseline $\quad 57$

7. Average difference scores (Experiment 1) 58

8. Orientation spectra of the different scalar conditions 61

9. Measures of horizontal perceptual change across magnitude of physical change 62

10. Each subject's horizontal difference score by KL divergence 62

11. Median difference scores (Experiment 2) 63

12. Measures of horizontal change across adaptation type 67

13. Median difference scores (Experiment 3) 68

14. Example of the filtering processing for Experiment $4 \quad 70$

15. Filters used for the inverted and enhanced conditions 71

16. Average pre and post adaptation median match values (Experiment 4) 73

17. Pre and post-test horizontal effect scores on average and for each subject $\quad 74$

18. Results of the enhanced natural and oblique dominant adaptation 76

19. The relationship between KL divergence and the difference scores 77

20. Average change across post-test period for all 3 global adaptation conditions $\quad 78$ 
21. Subjects pre and post test match values after adapting with no filter

22. S2's match values after adaptation to spatial frequency alteration

23. Subjects' pre and post test match values after adaptation to a vertical decrement 83

24. S2's matches after adaptation to vertical decrement with a 30 min delay in light 84

25. Subjects' match values after adaptation with oblique enhancement 85

26. Post-adaptation difference scores for S1 and S2 adaptation to vertical and horizontal 92

27. Post-adaptation difference scores for $\mathrm{S} 2$ adaptation to $45^{\circ}$ and horizontal 93

28. Variability across orientation pre and post adaptation to an isotropic environment 95

29. Likelihood and measurement distributions pre and post adaptation 97

30. Sample of every three degrees from natural scenes and the filtered images 98

31. Experimentally obtained bias and model fits 101

32. Model using the isotropic and the natural distribution as the prior 102 


\section{INTRODUCTION}

Almost since its inception, vision science has been concerned with the processing of lines at different sizes and orientations. Lines are important for visual processing for many reasons, primarily because $2 \mathrm{D}$ lines on the retina correspond to $3 \mathrm{D}$ edges in the real world. Lines and edges of different sizes and orientations are the elementary building blocks of the natural world and, as was discovered later, are not-coincidentally the fundamental encoding mechanisms for the cortical processing of images (Hubel \& Weisel, 1959, 1962). For the past decade, Essock and colleagues have been investigating the relationship between the processing of orientation by the human visual system and how this processing relates to the orientation content of the natural environment (Essock, DeFord, Hansen, \& Sinai, 2003; Essock, Haun, \& Kim, 2009; Hansen \& Essock, 2004; Hansen \& Essock, 2005; Hansen \& Essock, 2006; Haun \& Essock, 2010; Kim, Haun, \& Essock, 2010). For almost 100 years we have known that humans see cardinal orientations (horizontal and vertical) better than oblique orientations (Appelle, 1972; Campbell \& Kulikoski, 1966; Corwin, Moskowitz-Cook, \& Green, 1977; Emsley, 1925; Essock, 1982; Hamblin \& Winser, 1927; Higgins \& Stultz, 1948; Leibowitz, 1953; Shlaer, 1937). Many authors have also noted that natural scene statistics show a relationship to this differential visual processing of orientations (Coppola, Purves, McCoy, \& Purves, 1998; Hansen \& Essock, 2004; Keil \& Cristobal, 2000; Switkes, Mayer, \& Sloan, 1978). More recent work by Essock and colleagues has 
determined that orientation processing is more complex than it once appeared; when presented in a broadband, naturalistic context, human perception of horizontal orientations is actually worst and oblique orientations are perceived best (Essock,et al., 2003; Essock, Haun, \& Kim, 2009; Hansen \& Essock, 2004, 2005, 2006; Haun \& Essock, 2010; Kim, Haun, \& Essock, 2010). This finding has further extended the relationship between visual encoding and natural scene statistics as the differential perception of orientations in broadband scenes closely matches their differential representation in the natural environment (Essock, Haun, \& Kim, 2009; Haun \& Essock, 2010). However, the original hypothesis that this relationship may have evolved across millennia in order to make the visual system an efficient information-transmitting system is now being called into question by research showing the modification of orientation perception by exposure to altered environments (Bao \& Engel, 2012; Zhang, Bao, Kwon, He, \& Engel, 2009) and studies showing a later development of adult-like orientation processing (Ellemberg, Hansen, \& Johnson, 2012; Ellemberg, St Jacques, St LouisDeschenes, Baillargeon-Blais, \& Labonte-Lemoyne, 2012). Contemporary work into the effects of adaptation on visual encoding of the natural environment has led me to the conclusion that the relationship between the statistics of the natural world and visual encoding is, in a way, much simpler than previously posited (Bao \& Engel, 2012; Girshick, Landy, \& Simoncelli, 2011; Zhang et al., 2009). This project examines how the statistics of the very recently viewed visual world affect the way that the visual brain processes information. The work presented comments not only on how the visual system effectively encodes its surroundings, but perhaps also extends to a more general theory of how the brain as a whole processes information in a probabilistic and predictable manner. 


\section{Orientation Processing: Oblique Effect}

Human perception of orientation is biased; that is, visual perception differs depending on the orientation of the stimulus and the context in which the oriented stimuli is presented. For simple stimuli consisting of one orientation and one spatial frequency (i.e., a sine wave grating), human visual performance is worst at oblique and best at cardinal orientations (Appelle, 1972; Campbell \& Kulikoski, 1966; Essock, 1982). This differential performance at cardinal and oblique orientations is termed the oblique effect and has been shown to exist in man and animals on many kinds of tasks including those at absolute threshold (contrast sensitivity), other detection tasks (Vernier acuity, resolution acuity), and tasks above detection threshold (reaction time, orientation discrimination, apparent contrast) (Appelle, 1972; Corwin, Moskowitz-Cook, \& Green, 1977; Essock, 1982). Not only do subjects need higher contrast at oblique orientations to detect the presence of a stimulus (contrast sensitivity), they also take longer to react to stimuli at oblique orientations than cardinal orientations (Appelle, 1972; Attneave \& Olson, 1967) Perceptually, obliquely oriented gratings have lower apparent contrast than horizontal or vertical gratings of the same physical contrast both at absolute threshold and above threshold (Essock, 1982).

The more general oblique effect has been broken down into two classes based on the presumed mechanisms behind each effect (Essock, 1980). The class I oblique effect is found on tasks of basic visual processing such as contrast threshold and Vernier acuity such as those described above (Essock, 1980). There also exists a class II oblique effect which is found on suprathreshold tasks in paradigms involving the encoding/memory of orientation subsequent to the level of initial sensory encoding (Essock, 1980). Class II 
oblique effects are found on tasks of recognition, identification, discrimination, and matching and are not related to a difference in sensitivity, but differential capabilities in later processing (Essock, 1980, 1982; Essock, Krebs, \& Prather, 1997; Prather, Krebs, Sinai, McCarley, \& Essock, 1995). It is somewhat difficult to distinguish whether a specific task involves class I or II oblique effects (or both) as even tasks in which stimuli are presented simultaneously still rely on a difference in memory for orientations rather than just a bias in the initial visual encoding (Essock, 1980; Essock, Krebs, \& Prather, 1997). Both class I and class II oblique effects are found in children and adults, starting with infants as young as 6 weeks of age (Essock \& Siqueland, 1981; Leehey, MoskowitzCook, Brill, \& Held, 1975; Quinn \& Bomba, 1986; Quinn, Siqueland, \& Bomba, 1985).

The oblique effect has been tied to the number of orientation-tuned neurons in early areas of visual cortex (V1) (DeValois, Yund, \& Hepler, 1982; Kennedy, Martin, Orban, \& Whitteridge, 1985; Li, Peterson, \& Freeman, 2003; Liang, Shen, \& Shou, 2007; Mansfield, 1974; Mansfield \& Ronner,1978; Shen, Liang, \& Shou, 2008; Shen, Tao, Zhang, Smith, \& Chino, 2014; Wang, Ding, \& Yunokuchi, 2003), the amplitude of the response from cardinally tuned neurons (Maffei \& Campbell, 1970; Sun et al., 2013), tuning differences between cardinally and obliquely tuned neurons (Dragoi Turcu, \& Sur, 2001; Li, Peterson, \& Freeman, 2003), and the cortical space devoted to processing different orientations (Chapman \& Bonhoeffer, 1994; Chapman, Stryker, \& Bonhoeffer, 1998; Grabska-Barwinska, Distler, Hoffman, \& Jancke, 2009; Huang et al., 2006). The evidence for the differential representation of cardinally tuned cells in primary visual areas of animals has come from multiple sources (Chapman \& Bonhoeffer, 1994; Chapman, Stryker, \& Bonhoeffer, 1998; Coppola et al., 1998; DeValois, Yund, \& Hepler, 
1982; Huang et al., 2006; Kennedy et al., 1985;Mansfield 1974; Mansfield \& Ronner, 1978; Shen, Liang, \& Shou, 2008), but studies in humans have led to mixed results (Freeman, Brouwer, Heeger, \& Martin, 2011; Furmanski \& Engel, 2000; Kamitani \& Tong, 2006; Koelewijn, Dumont, Muthukumaraswamy, Rich, \& Singh, 2011; Maffei \& Campbell, 1970; Nasr \& Tootell, 2012; Proverbio, Esposito, \& Zani, 2002; Serences, Saproo, Scolari, Ho, \& Muftuler, 2009; Swisher et al., 2010; Sun et al., 2013; Yacoub, Harel, \& Ugurbil, 2008). Mansfield (1974) was the first to demonstrate an orientation bias in the number of cells tuned to cardinal orientations by recording single units' optimal orientation and receptive field (RF) eccentricity in rhesus monkey primary visual cortex. This anisotropy in the number of cells that respond to different orientations has also been found in macques (DeValois, Yund, \& Hepler, 1982). More neurons preferring cardinal orientations compared to oblique orientations were found within the central 2 degrees of the visual field (parafoveal regions outside of this did not show any anisotropy) (Mansfield \& Ronner, 1978). Li, Peterson, and Freeman (2003) also found more cardinally tuned cells than obliquely tuned cells among the simple cell population in cat primary visual cortex. In more recent studies, Shen and colleagues (2008; 2014) found evidence of a greater number of cardinally tuned cells in the foveal region of V2, but not V1 of cats (area 17) and macaques. Generally speaking, most studies have found a numerical anisotropy wherein cardinally tuned cells are overrepresented in primary visual cortex of cats (Li, Peterson, \& Freeman, 2003; Liang, Shen, \& Shou, 2007; Shen, Liang, \& Shou, 2008) although the results from primates are mixed and suggest that the anisotropy may exist at higher levels (i.e. V2, see Shen et al., 2014 for review). 
The anisotropy of quantity is coupled with an anisotropy of tuning; although peak firing rate of cardinal and obliquely tuned cells are the same, cardinally tuned cells are more sharply tuned than obliquely tuned cells (Dragoi, Turcur, \& Sur, 2001; Li, Peterson, \& Freeman, 2003). Dragoi, Turcu, and Sur (2001) also found that cardinally tuned cells tend to have iso-oriented input, while obliquely tuned cells receive input from areas of larger cortical spread. Since cardinally tuned cells have more specific inputs, their orientation tuning should be less affected when adapted to a stimulus that is oriented away from their preferred orientation (Dragoi, Turcu, \& Sur, 2001). Adapting orientation-tuned cells in cat primary visual cortex to oriented gratings resulted in strong changes in the orientation preferences of obliquely tuned neurons, but much smaller changes in cardinally tuned neurons. Thus, the authors concluded that cardinally tuned neurons are more stable in their preferred orientations and the percepts that they induce (Dragoi, Turcu, \& Sur, 2001). This stability and narrow tuning, along with differential population representation, may further allow the visual system to minimize changes in perception due to adaptation by providing a "frame of reference" along cardinal axes (Dragoi, Turcu, \& Sur, 2001). In other words, cells tuned to cardinal orientations are more specific in their tuning and less susceptible to changes which could allow the visual system to maintain a 'true' vertical and horizontal from which other orientations can be referenced. However, not all studies investigating the tuning responses of primary visual cortical neurons have found this stability at the cardinal orientations (Dragoi, Sharma, \& Sur, 2000; Nagai, Suzuki, \& Wang, 2007); more work is needed to fully investigate the frame of reference hypothesis. 
Optical imaging studies have further supported the existence of a cardinal bias in neural population tuning of orientation in animals. Optical imaging on newborn ferrets by Chapman and Bonhoeffer (1994; Chapman, Stryker, \& Bonhoeffer, 1998) revealed that cardinal response areas were significantly larger than oblique response area across postnatal days 32 to 42 . Other optical imaging studies found similar evidence of an increased amount of processing devoted to cardinal orientations and provided evidence that the orientation anisotropy also exists at higher levels (area 21a in cat cortex) in the visual system beyond primary visual cortex (Huang et al., 2006). The existence of higher level anisotropies may indicate that feedback from these higher areas plays a key role in shaping the anisotropy in lower order cortex (Huang et al., 2006). However, as single cell recordings and optical imaging studies are limited to relying on animal models, the exact implications of these findings are unclear and may not be applicable to human visual areas (i.e., V1). Whether occurring in primary visual areas or higher order visual areas, it does seem clear that animal models indicate an overrepresentation of cardinal contours in early areas of visual cortex (Chapman \& Bonhoeffer, 1994; Chapman, Stryker, \& Bonhoeffer, 1998; Coppola et al., 1998; DeValois, Yund, \& Hepler, 1982; Grabska-Barwinska, Distler, Hoffman, \& Jancke, 2009; Huang et al., 2006; Kennedy et al., 1985; Maffei \& Campbell, 1970; Mansfield 1974; Mansfield \& Ronner, 1978; Li, Peterson, \& Freeman, 2003; Shen, Liang, \& Shou, 2008; Shen et al., 2014; Wang, Ding, \& Yunokuchi, 2003).

In humans, the differential representation of orientations in cortex has proved less conclusive; while some studies show a cardinal bias (Aspell, Wattam-Bell, Atkinson, \& Braddick, 2010; Furmanski \& Engel, 2000; Furmanski, Schluppeck, \& Engel, 2004; 
Maffei \& Campbell, 1970; Proverbio, Esposito, \& Zani, 2002; Serences et al., 2009; Swisher et et al., 2010; Sun et al., 2013) others show no bias (Kamitani \& Tong, 2005; McDonald, Mannion, \& Clifford, 2012; Yacoub et al., 2008) or mixed results (Koelewijn et al., 2011). The amplitude of response to cardinal gratings does appear to be greater than that evoked by oblique gratings in human visual cortex. Using ERP, the amplitude evoked by a passively-viewed, drifting grating with cardinal orientations is larger than that of oblique orientation (Maffei \& Campbell, 1970) and there are larger P1 and P3 components for cardinal orientations when subjects are performing an orientation identification task (Proverbio, Esposito, \& Zani, 2002). Using MEG, a technique which has better spatial resolution than ERP and better temporal resolution than fMRI, Koelewijin and colleagues (2011) found an early (80ms) reverse oblique component in medial part of visual cortex and a relatively later $(120 \mathrm{~ms})$ classic oblique effect component. The earlier inverse oblique component may reflect the broader tuning widths of the obliquely tuned cells and be later overruled by the later component (Koelewijn et al., 2011). MRI evidence in human processing of orientation has been particularly mixed. Using discrete grating patches experimenters found a larger BOLD response for cardinal orientations in V1, but not in V2 and V3 (Furmanski \& Engel, 2000). More recently, Kamitani and Tong (2006) were able to successfully decode orientation perception from the BOLD signal of subjects, but found no preferential tuning across orientation. However, using a higher resolution fMRI to measure the orientation tuning in V1, Serences and colleagues (2009) did find a higher proportion of voxels which responded maximally to horizontal orientation. The most recent study to date suggests that the mixed fMRI evidence collected in the past led to unclear results purely because 
of coarse sampling techniques (Sun et al., 2013, Freeman, Heeger, \& Merriam, 2013). Using both a continuous and periodic stimuli presentation paradigm, Sun and colleagues (2013) found more voxels in V1 preferring horizontal and vertical orientations. Thus, there appears to be a cardinally dominant anisotropy of response profiles in human V1, although this predominance may not extend to higher visual cortical areas.

Whether the cause of the oblique effect is a difference in the number of cells, the amplitude of the response, the tuning properties of cell populations, or the cortical area devoted to cardinal orientation processing, the effects of the differential cortical treatment of orientations are present from a very early age, possibly birth. Human infants showed an oblique effect as early as 6 weeks of age with better visual acuity thresholds for cardinal orientations than oblique orientations (Atkinson, Hood, Wattam-Bell, Anker, \& Tricklebank, 1988; Leehey, et al., 1975). Visual evoked potential (VEP) tests on human infants aging 2 to 11 months old indicate the amplitude of vertically oriented stimuli was significantly larger than oblique gratings while the latencies were significantly shorter (Sokol, Moskowitz, \& Hansen, 1987). Single unit recordings from macaque infants (4-8 weeks of age) also show a numerical bias in the number of cells tuned to cardinal orientations in visual area V2 (Shen et al., 2014). The numerical and tuning biases mentioned above makes the processing of horizontal orientations less susceptible to perceptual change and also probably contributes to the oblique effect in that a greater number of cells tuned to cardinal orientations should lead to a stronger response to cardinal gratings (Dragoi, Sharma, \& Sur, 2000; Hansen \& Essock, 2006; Leventhal \& Hirsch, 1977; Orban \& Kennedy, 1981). Though there may not be a simple and 
straightforward hypothesis of how the oblique effect anisotropy emerges physiologically, it is clear that there is preferential processing of cardinal orientations in cortex.

\section{Orientation Processing: Horizontal Effect}

All of the above oblique-effect phenomena have been found using simple, narrowband stimuli, and most often single, sine-wave gratings; the oblique effect is found on tasks whose stimuli are narrowband, both in terms of spatial frequency and orientation content. However, the natural visual world is obviously broadband in nature, consisting of multiple spatial frequencies and orientations. Therefore, Essock, DeFord, Hansen, and Sinai (2003) proposed using broadband noise stimuli to study human perception of orientation. Subjects were asked to adjust a test stimulus to "match the perceived 'strength' or 'salience' of the oriented structure" that they perceived in a standard (comparison) stimulus. Subjects needed a higher amplitude of physical orientation content in horizontal and vertical test stimuli to perceptually match the standard than they needed in oblique stimuli (Essock et al., 2003). That is, with such broadband stimuli, a nearly-opposite orientation anisotropy is found from the traditional oblique effect: stimuli at cardinal orientations are perceived less well than oblique orientations with horizontal being perceived the worst and vertical intermediate (between horizontal and oblique). Following the naming convention of the oblique effect, this broadband orientation bias has been termed the 'horizontal effect' (Essock et al., 2003, Essock et al.,2009; Hansen \& Essock, 2004; Haun \& Essock, 2010). The horizontal effect, like the oblique effect, is found on absolute detection tasks (broadband masking of oriented Gabors) and tasks above detection threshold (matching the apparent contrast of two broadband, oriented patterns.) (Essock et al., 2003; Essock, Haun, \& Kim, 2009; Hansen \& Essock, 2006). 
Furthermore, the horizontal effect is found not only with broadband noise stimuli, but naturalistic images as well (Hansen, Essock, Zheng, \& DeFord, 2003; Hansen \& Essock, 2004). When stimuli consist of natural images whose amplitude content has been artificially altered to contain differential amounts of either cardinal or oblique orientations, the horizontal effect persists (Hansen \& Essock, 2004). Using Glass patterns, a stimulus consisting of numerous dots paired into a global structure (and thus having a broader orientation spectrum), researchers found an 'inverse oblique effect' wherein subjects show greater sensitivity to oblique orientations over cardinal orientations (Wilson, Loffler, Wilkinson, \& Thistlethwaite, 2001). Other researchers have found a similar decreased sensitivity to horizontal orientations using such Glass pattern stimuli; subjects show an increased threshold for detecting coherent, implied motion when it is horizontally compared to vertically oriented (Nankoo, Madan, Spetch, \& Wylie, 2012). Using stimuli which varied from narrowband to broadband in nature, Hansen and Essock (2006) measured subjects' perceptual matches to a comparison stimulus. The results indicated that there is a transition from an oblique to a horizontal effect as stimuli include both more spatial frequencies and orientations (Hansen \& Essock, 2006).

There has been significantly less study of the physiological underpinnings of the horizontal effect than of the oblique effect. Specifically, only one study to date has directly sought to uncover the neural mechanisms of the horizontal effect (Yang et al., 2012). Though this is the case, many authors have found intriguing evidence as to the neural mechanisms underlying the horizontal effect even though they were not directly seeking it. Some fMRI evidence has actually contradicted earlier reports of increased 
activation in response to cardinal gratings: using sinusoidal gratings, authors found the greatest activation in all primary visual areas (V1, V2, V3, and V3a) to oblique stimuli and the least activation to horizontal gratings (Mannion, McDonald, \& Clifford, 2010a). This pattern of activity would lead to a flattened/whitened representation of the natural world (see below); although the number of horizontal cells is greater, the activated response is lower. Though these stimuli were not broadband in nature, this study indicates that what may seem clear-cut underpinnings of the oblique effect are in fact much more complicated.

Another study which aimed to find evidence of the oblique effect via ERP recordings may have used stimuli that actually evoked a horizontal effect; the stimuli used were simple lines, but consisted of multiple orientations and sharp edges which, in terms of Fourier power, contain multiple spatial frequencies (spatially broadband). These stimuli elicited larger P300 amplitudes for oblique lines than for cardinal stimuli (Heinrich, Aersten, \& Bach, 2008). Although the authors attempted to qualify these results by claiming that, statistically speaking, obliques are 'oddball' stimuli and therefore elicit greater P300 amplitude, it could also be that the stimuli they were using were actually causing more of a horizontal effect than an oblique effect. Comparable results were obtained using functional near-infrared spectroscopy (fNIRS) to measure changes in oxygenated hemoglobin in the occipital cortex in response to oriented gratings: there were larger amplitudes in the left occipital lobe in response to oblique than horizontal or vertical gratings (Sun et al.,2013). Similarly, using Glass patterns, consisting of structured, random dots (broad orientation spectrum), Mannion and 
colleagues (2010b) found a greater fMRI BOLD response to vertical orientations than obliques in V1 (although horizontal showed no differences from oblique orientations).

The only study to directly address the question of the physiological cause of the horizontal effect was conducted while recording ERPs. Subjects performed an orientation-identification task with broadband noise stimuli with different predominant orientations very similar to those used previously by Essock and colleagues (Yang et al., 2012). These stimuli evoked a psychophysical horizontal effect while inducing some interesting ERPs. The P2 component, which occurs at 200ms post stimulus, showed significant differences between cardinal and oblique gratings such that horizontally and vertically oriented stimuli elicited larger P2 amplitudes than obliquely oriented stimuli (Yang et al., 2012). This component is much later than traditional oblique effect components indicating to these authors that it was related to the horizontal effect as the processing involved in that effect should occur at later stages of processing than the oblique effect (Yang et al., 2012). The P300 component was also orientation specific, showing larger and earlier amplitudes for cardinal gratings than for oblique gratings. In contrast to traditional oblique effect findings, this study showed a longer P3 latency for cardinally oriented noise images than obliquely oriented ones. The authors related the longer latencies back to the behavioral horizontal effect: slower reaction times and poorer identification of cardinal compared to oblique broadband noise stimuli (Yang et al., 2012). Although for the two main component differences (P2 and P3 amplitudes) cardinal gratings still induced larger ERP amplitudes (like in traditional oblique effects), the differences for orientation occurred at later stages than classic oblique effect differences (Yang et al., 2012). These authors concluded that the horizontal effect, then, 
also occurs at later stages of processing than the traditional oblique effect, perhaps due to the need for pooling of the overall population response to multiple orientations and spatial frequencies present in horizontal effect-inducing stimuli. To date, no studies have looked at the development of the horizontal effect, although reports on gain control and other broadband tuning biases (as discussed below) may indicate that the horizontal effect does not affect visual processing until much later in development than the oblique effect.

In response to the horizontal effect of human perception, researchers have developed an adapted model of gain control processes in early visual cortex (Hansen \& Essock, 2004). Gain control is known to occur at early levels of the visual hierarchy to accommodate wide ranges of stimulus input (Ohzawa, Sclar, \& Freeman, 1985). A single cell in the earliest levels of visual cortex is tuned to encode one variety of all the possible local properties of a visual stimulus (i.e., hyper columns in V1 made up of cells specifically tuned to different orientations and spatial frequencies). However, this tuning must also be relevant to the biases present in the global stimulus. That is, the response of a tuned (orientation or spatial frequency) filter over one point in space needs to be compared to the overall response of all tuned filters to all spatial aspects of the stimuli presented in order to consider the relative impact of one specific visual feature. By comparing each filter response to the population, the response of tuned neurons changes to match the overall mean of the stimulus (Brenner, Bialek, \& de Ruyter van Steveninck, 2000; Clifford et al., 2007; Foley, 1994; Ohzawa, Sclar, \& Freeman, 1985). While simple, narrowband stimuli generate responses from only a few orientation-tuned filters, broadband stimuli generate responses from many more filters. The response to a stimulus with many spatial frequency and orientation components should be normalized by the 
response of similar filters (in terms of orientation and spatial frequency). Since there are more horizontal components in a natural image, if cardinal and oblique orientations were weighted equally, the horizontal and near-horizontal filters would be driven more than oblique filters. On the contrary, the gain control model proposed by Essock and colleagues (see Hansen \& Essock, 2004) explains the difference in perception between the oblique effect and the horizontal effect based on anisotropic suppression. When simple gratings are used as stimuli (such as in the oblique effect), the differential representation of cardinally tuned neurons in cortex leads to a greater response to these than oblique orientations and the suppressive normalization pool contributes less due to a lack of other stimulus components. When broadband stimuli are used, the normalization pool has a much more significant impact on the response, suppressing the more prevalent orientations (horizontal and vertical) more than the obliques.

It has been suggested that the population response making up the normalization pool in early levels of the visual cortex has differential suppressive weights associated with different orientations such that horizontal (and to a lesser extent vertical) orientations are suppressed more than oblique orientations (Essock, Haun, \& Kim, 2009; Hansen \& Essock, 2004; Haun, Hansen, \& Essock, 2006; Haun \& Essock, 2010; Kim, Haun, \& Essock, 2010; see also Schwartz \& Simoncelli, 2001; Wainwright, 1999). This anisotropic suppression leads to the perceptual effect wherein horizontal orientations are seen less well than obliques in broadband patterns (i.e., the horizontal effect). To measure the timing of orientation-tuned suppression, Haun, Essock, and Kim (2009) used a narrowband target stimulus ( 8 cycles/degree-CPD) at four orientations (vertical, 45 , horizontal, and 135) and a broadband mask at the same orientations. (The horizontal 
effect is greatest around 8 CPD; Haun \& Essock, 2010.) Effectively, long stimulus onset or offset asynchronies (SOA) correspond to two separate stimuli: a broadband stimulus that is separate from the narrowband target while shorter SOAs are closer to actual masking as if the mask and target were presented simultaneously. For very long SOAs, an oblique effect was found with thresholds for detecting the 8 CPD grating being lowest at the cardinal orientations and higher at obliques. However, for shorter SOAs, a horizontal effect was found such that thresholds were higher for the cardinal orientations than the obliques. This horizontal effect was asymmetric in that backward masking produced a larger horizontal effect than forward masking. Models of masking from previous literature were used with an added anisotropic weighting factor in the mask response $(M)$ (Foley, 1994). The model of sensitivity of the gain control mechanism is divisive such that the response is divided by a semi-saturation constant, $A_{M} K_{0}$, and the content of the mask, $M$ (equation 1).

$$
S(s)=\left(\frac{1}{c}\right) \sum_{t} R(t, s)_{\text {out }}=\sum_{t}\left(\frac{R(t)}{\left(A_{M} K_{0}+M(t, s)\right)}\right) \quad \begin{aligned}
& \text { Equation 1. from } \\
& \text { Essock, Haun, \& Kim, } \\
& 2009 .
\end{aligned}
$$

The semi-saturation constant $A_{M} K_{0}$, is composed of two static components: $A_{M}$ which represents the change in the adaptation state of the detecting mechanism due to the mask and $K_{0}$ which represents the 'absolute' semi-saturation constant and is inherently anisotropic, weighting oblique orientations more heavily than horizontal and vertical. $K_{0}$ weights horizontal and vertical orientations equally and thus induces the oblique effect (larger response to horizontal and vertical) in the absence of a mask component. Using the psychophysical data as ouput showed that the model produced anisotropies both in the mask component $M$ and in the semi-saturation constant modifier $A_{M}$ (Essock, Haun, \& 
Kim, 2009). The $A_{M}$ factor is equal to 1 when no mask is present and free to vary when a mask is present, something like an adaptation factor (Essock, Haun, \& Kim, 2009). When there is a broadband mask, $A_{M}$ weights horizontal orientations the same as vertical orientations working against (and, with more mask components, overcoming) the oblique effect of the $K_{0}$ component. The weighting factor $(w)$ in the mask $(M)$ contributes to the horizontal effect because it weights horizontal more than obliques and vertical least of all.

$$
\begin{array}{ll}
M_{\text {onset }}(t, s)=w\left(1-e^{-\frac{L-s}{\tau_{c n}}}\right), 0 \leq t-s \leq 50, & \text { Equation 2. From } \\
\text { Essock, Haun, and } \\
\text { Kim, 2009 }
\end{array}
$$

When mask and target are close in time, these two factors are taken together and a horizontal effect is produced: worst performance at horizontal and best at obliques with vertical intermediate (Essock, Haun, \& Kim, 2009). The horizontal effect can only appear at small SOAs because the $w$ factor is tied to the dynamic gain control component, $M$, and masking (i.e., $M$ ) is so short lived. This dynamic component is presumably influenced by the current environment of the observer adjusting perceptual salience rapidly based on ongoing changes in the orientation content of the natural world. There is some variability between observers as to where in between horizontal and oblique performance vertical performance falls for a given broadband mask and this variability may be caused by relative differences in the two weighting factors for different individuals (Essock, Haun, \& Kim, 2009).

\section{Natural Scenes: Orientation Content}

Interestingly, the differential suppression across orientation in cortex matches well with content biases in the natural environment: natural scenes have the most oriented 
content at horizontal, second most at vertical, and least at the obliques (Girschik, Landy, \& Simoncelli, 2011; Hansen \& Essock, 2004; Keil \& Cristóbal, 2000). Some work has failed to show a difference in horizontal compared to vertical energy, but all studies have found more combined cardinal energy than oblique energy in the natural world (Baddeley \& Hancock, 1991; Coppola et al., 1998; Hancock, Baddeley, \& Smith, 1992; Girschik, Landy, \& Simoncelli, 2011; Hansen \& Essock, 2004; Keil \& Cristóbal, 2000; Oliva \& Torralba, 2001; Switkes, Mayer, \& Sloan, 1978 ). Furthermore, all types of natural scenes show this anisotropic pattern including outdoor scenes with manmade content, those without manmade content, and indoor scenes (Coppola et al., 1998; Switkes, Mayer, \& Sloan, 1978). Moreover, different methodologies (optical Fourier analysis, digital FFT, PCA, and higher order autocorrelation analyses) used for assessing the amount of oriented structure in the visual world all yield similar results (i.e., more amplitude content at cardinal orientations) (Baddeley \& Hancock, 1991; Coppola et al., 1998; Hancock, Baddeley, \& Smith, 1992 Hansen \& Essock, 2004; Switkes, Mayer, \& Sloan, 1978).

Several of the authors who have studied the orientation distribution in the environment have noted that there may be a relationship between the natural, physical anisotropy and that of visual performance (Coppola et al., 1998; Hansen \& Essock, 2004; Keil \& Cristobal, 2000; Switkes, Mayer, \& Sloan, 1978). Although many of these authors attempted to relate the natural anisotropy to the oblique effect, hypothesizing that the predominance of cardinal orientations in the environment is the reason behind their superior processing, the discovery of the horizontal effect has led others to the opposite conclusion: namely, that, in broadband contexts like natural scenes, visual performance is 
worse at horizontal orientations (Hansen \& Essock, 2004, 2005). Furthermore, Essock, Haun, and Kim (2009) noted that perceptual biases of the horizontal effect closely match those found in natural scenes; the percentage of anisotropic content in the environment is a fairly accurate match to the percentage of anisotropic suppression induced by the cortex. In other words, the amount of suppression of horizontal content induced by visual processing is approximately equal to the percentage that these orientations are dominant in natural scenes (Essock, Haun, \& Kim, 2009; Hansen \& Essock, 2005). Performance on a masking task showed horizontal thresholds $11 \%$ higher than vertical, vertical thresholds $15 \%$ higher than obliques and horizontal $28 \%$ higher than obliques. A large database of natural scenes similarly showed that horizontal content is, on average, $7 \%$ higher than vertical, vertical is $12 \%$ higher than obliques, and horizontal is $20 \%$ higher than obliques (Hansen \& Essock, 2004). By suppressing horizontal orientations more than oblique orientations, our perceptual biases serve to emphasize what is least present in our natural environment rather than what is most present. Similar to the way we perceive spatial frequency (see below), orientation biases in a scene seem to be "whitened" by the visual system.

\section{Natural Scenes: Efficiency and Bayesian Encoding}

The reciprocal relationship between natural scene regularities and visual processing biases suggests that the biases present in the visual cortex serve to 'undo' or whiten those present in the environment (Atick \& Redlich, 1990, 1992; Barlow, 1989; Graham, Chandler, \& Field, 2006). Not only does the brain whiten orientation content, but it also differentially processes size to 'undo' the typical relationship between amplitude and spatial frequency present in natural scenes (Field \& Brady, 1997; Hansen 
\& Essock, 2005; Simoncelli \& Olhausen, 2001; Tolhurst, Tadmor, \& Chao 1992; van der Schaaf \& van Hateren, 1996). Previous researchers have found that the amplitude of content in natural scenes tends to fall off in a regular and predictable pattern with spatial frequency: amplitude $=1 /$ frequency $^{\alpha}(\alpha$ or slope of the log-log plot of amplitude by spatial frequency =-1) (e.g. Field 1987; Tolhurst, Tadmor, \& Chao 1992). Ostensibly not by chance, human visual processing of naturalistic $1 / \mathrm{f}^{\alpha}$ stimuli also tends to be biased with higher stimuli discrimination and identification rates occurring with naturalistic slopes than in images whose slope falls outside the typical range (Bex, Solomon, \& Dakin, 2009; Field \& Brady, 1997; Hansen \& Hess, 2006; Johnson, Richard, Hansen, \& Ellemberg, 2011; Parraga, Troscianko, \& Tolhurst, 2000; Tolhurst, Tadmor, \& Chao, 1992). Visual processing of spatial frequency in naturalistic images seems to indicate that the lower spatial frequencies, which are more prominent in natural scenes, are suppressed (Bex et al., 2009; Haun \& Pelli, 2013; Webster \& Miyahara, 1997). Not unlike orientation processing, the early stages of spatial frequency processing also seem to be tuned to the spatial frequency content of natural scenes (Atick \& Redlich, 1990, 1992; Graham, Chandler, \& Field, 2006).

Based on the above, it seems that the visual system is optimized to process natural scenes such that it produces whitened outputs in response to biased inputs, effectively increasing information transmission (Attneave, 1954; Barlow, 1961, 1989). The statistical regularities in natural images make them very inefficient stimuli as there are strong correlations between neighboring regions and thus a preponderance of redundant information (Atick \& Redlich, 1992). The visual system acts like an effective information transfer system by minimizing information carried from multiple units about 
the same proportion of the environment (Atick \& Redlich, 1990, 1992). The gain control processes which suppress the most prevalent content in the environment in effect improve the efficiency of the visual brain by de-emphasizing common content and reciprocally emphasizing content that is rarer. This efficient processing of information decreases the information that was redundant in the incoming natural image equalizing or whitening the signal (Essock, Haun, \& Kim, 2009; Hansen \& Essock, 2004, 2006).

Thus, early visual areas appear to efficiently process biases inherent in our natural environment, both in terms of orientation and spatial frequency. The distribution of orientations in the environment is 'anti-correlated' with activity in V1 suggesting that early visual processing is predictive of the natural viewing environment (Clifford, Mannion, \& McDonald, 2011). The regular patterns of orientation and spatial frequency distribution present in our natural environment are processed most efficiently by the human visual brain as shown by preferential behavioral treatment of naturalistic stimuli (Bex, Solomon, \& Dakin, 2009; Hansen \& Essock, 2004; Hansen \& Hess, 2006; Johnson et al., 2011). Moreover, the psychophysical correlations to natural stimuli have reliable, if somewhat understudied, physiological underpinnings as well (Clifford, Mannion, \& McDonald, 2011; Yang et al., 2012). Both whitening processes can be well modeled by assuming that observers have something like a Bayesian prior probability distribution of expected orientations (and presumably spatial frequencies) that is based on the natural anisotropy (Girschik, Landy, \& Simoncelli, 2011).

Girschik, Landy, and Simoncelli (2011) found that an observer's internal prior probability distribution of orientations in the environment is a good match to the environmental distribution. The study recovered the observers' priors from 
measurements of perceptual bias and variability on a task which asked observers to compare the global average orientations of two arrays of Gabor patches with variable noise levels (amount of different orientations presented in individual Gabors). If subjects had a non-uniform prior it would cause them to bias their estimation of whether the test stimulus was, on average, oriented clockwise or counterclockwise of the comparison stimulus (if the prior was uniform across orientation, no bias would be observed). The bias can be computed by comparing the mean physical orientation difference between two stimuli which are perceived as equal in average orientation (Girschik, Landy, \& Simoncelli, 2011). All subjects showed a bias towards cardinal orientations in high noise stimuli that subsequently led to a bimodal recovered prior with peaks at the two cardinal orientations. As mentioned above, the environmental distribution of orientations is quite similar with primary peaks at horizontal and vertical orientations (Coppola et al., 1998; Hansen \& Essock,, 2004; Switkes, Mayer, \& Sloan, 1978). In this study, the environmental distribution was computed by calculating the probability distribution based on a histogram of 'highly oriented' regions created by convolving rotationinvariant filters at a spatial scale matched to human peak sensitivity (2-5 CPD) with a large database of photographs of natural scenes which contained no carpentered content (Girshick, Landy, \& Simoncelli, 2011). Interestingly, the authors found that a Bayesian encoder-decoder model had similar variability and bias whether they used the recovered prior (from observer data) or the environmental distribution (purely natural scenes) for the model on the same task used with human observers (Girshick, Landy, \& Simoncelli, 2011). Performance with the model closely resembled human behavior even when the prior that was used was simply based on the environmental distribution. Therefore, the 
authors concluded that the human observers used prior knowledge of the orientation statistics of the environment in their perceptual task (Girschik, Landy, \& Simoncelli, 2011).

There is converging evidence that a Bayesian decoder may in fact be the best way of interpreting the population code of neurons in cortex (Fiser, Berkes, Orban, \& Lengyel, 2010; Mamassian, Landy, \& Maloney, 2002; Weiss, Simoncelli, \& Adelson, 2002). On the one hand, the tuning curves of neurons could simply be adapted by gain control and pooled into a population code in a 'winner takes all fashion.' This kind of coding relies on the peak response of the neuronal pool to decode the incoming stimulus (the stimulus is presumed to lie at the peak of the pooled distribution). However, a more accurate method involves taking into account the likelihood of the stimulus given noise corrupting the signal and using prior information about the probability of the stimulus in our environment to decode population information (Pouget, Dayan, \& Zemel, 2003). By including information from the whole likelihood (not just the peak firing rate or peak + noise) a Bayesian estimator provides a more accurate prediction of stimulus values than the population code alone (Beck et al., 2008; Pouget, Dayan, \& Zemel, 2003). Many studies have also indicated that humans perform perceptual tasks based on a combination of prior assumptions about the environment and the likelihood of a given stimulus value (e.g., Fiser \& Aslin, 2001, 2005; Turk-Browne, Scholl, Chun, \& Johnson, 2009). These studies suggest that subjects cannot merely be relying on the peak of a distribution to give reliable responses but must instead have access to the combined likelihood and prior.

Despite the vast amount of modeling showing matches between human performance and Bayesian observers, there is little corresponding neurophysiological 
work partially due to the problem representing uncertainty in neural networks (Fiser et al., 2011). All processing involves the fusion of information from multiple sources (i.e., the color and shape of an object plus our memory of objects we have seen in the past) and the system must be able to quantify the uncertainty of these multiple sources of information. Representing uncertainty in neural networks is important because uncertain sources of information should be relied upon or weighted less than more certain sources of information (Fiser et al., 2011). The representation of uncertainty is probabilistic and related to our prior experience with the environment. Most neural models have only been able to demonstrate that the known properties of $\mathrm{V} 1$ encoders are a good match to model predicted units rather than directly testing physiological units. More recent evidence has found a possible neural basis for probabilistic coding by recording from actual units. These studies have found that spontaneous neural activity in the population may not be noise (as once hypothesized); it is higher in particular areas and correlates with stimulusevoked activity, suggesting that spontaneous activity may have a functional role (Berkes, Orban, Lengyel \& Fiser, 2011; Fiser et al., 2013). The representation of the prior probability distribution in the spontaneous activity of the visual cortex fits with the Bayesian encoder-decoder model.

Under the framework of a Bayesian representational account of neural population coding, spontaneous activity could represent the prior. If a statistical model is appropriately describing its input, the prior distribution should closely match the average posterior distribution; a well-developed prior will be reliable and thus match the posterior (Berkes et al., 2011). That is, the neural representation of the prior probability distribution of an incoming stimulus should be closely correlated with the activity evoked 
by the actual stimulus itself. If spontaneous activity represents prior expectations in an internal model, then it should correlate with stimulus-evoked activity, and this is exactly what has been found in early visual areas of ferrets and cats (Berkes et al., 2011; Kenet, Bibitchkov, Tsodyks, Grinvald, \& Areli, 2003; Tsodyks, Kenet, Grinvald, \& Areli, 1999). The correlation between stimulus-evoked activity and spontaneous firing in ferret primary visual cortex increases with developmental age, but does not occur when the development of the visual system is disrupted by dark rearing (Berkes et al., 2011; Fiser et al., 2013). Furthermore, spontaneous activity is biased towards cardinal orientations: spontaneous activity that is correlated with a specific orientation show a strong bias towards cardinal orientations (Kenet et al., 2003). Although highly variable, spontaneous activity corresponding to one of the cardinal orientations (either horizontal or vertical) appeared on average $20 \%$ more often than that corresponding to the oblique orientations in area 18 of cat visual cortex (Kenet et al., 2003). If spontaneous activity represents the prior probability distribution in the environment, it should be weighted towards the cardinal orientations as they are predominant.

Although there is significant psychophysical evidence showing that humans are optimal learners and many studies have shown a relationship between environmental distributions of stimulus features and optimal observer priors, there is an inherent danger in matching the prior precisely to the environment. While using an environmental distribution to approximate an observer's prior may show better performance, it is unwise to tune the prior to perfectly match the environment because it risks being over-tuned (Feldman, 2013). Some of the information in the environment is actually noise. By fitting a model of observer performance exactly to the environmental distribution, we risk 
fitting in the noise as well as the 'true' data. Moreover, there is inherent uncertainty about future environments which one might encounter with different distributions of stimulus features (Feldman, 2013). Feldman (2013) argues, then, for a 'regularized prior:' one that is in between the ideal environmental state (ecological prior) and the maximum entropy prior (maximally noisy input). Simply by increasing the entropy (noise) of our modeled priors we can therefore more accurately represent the presumed internal state of observers (Feldman, 2013). Hence, the correlation between spontaneous activity and stimulus evoked activity should not be exact, but allow room for uncertainty/noise. Spontaneous activity may also play a role in driving the network close to states that are probable to be valid inferences once input arrives by priming specific cells making it easier for them to fire (Fiser et al., 2011). Fiser and colleagues (2011) hypothesize that spontaneous activity could also be involved in re-tuning synaptic weights during off-line periods (i.e. sleep), refining the internal model of the environment. It is likely that the Bayesian framework is a good model, then, for how population codes decode information in an efficient manner: to predict stimulus input values based on their likelihood as well as the probability of encountering them given known parameters in the environment rather than simply peak firing rates of neurons tuned to specific stimulus properties.

\section{Adaptation: Explanation of Perceptual Effects}

Adaptation processes may provide the efficient coding mechanism used to normalize the encoding process, change the likelihood, and maximize information transmission. Sensory adaptation refers to changes in the current perception or neural encoding of stimuli based on recent sensory stimulation (Greenlee \& Heitger, 1988; 
Webster, 2011). Stimulus-specific adaptation involves a decrease in response (perceptual or neural) to a repeated stimulus. A consequence of the decline in response induced by prolonged stimulation is that the sensory system will be at a continually low steady-state level. Rather than continually encoding the repeated stimulus at maximum response, the response decreases so that the current level of the system is closer to baseline. In this way, the response to any new stimulus will be enhanced relative to the decrease resulting from adaptation - that is, new stimuli are much more perceptually salient (Clifford et al., 2007; Kohn, 2007; Webster, 2011). Adaptation studies have added much to our understanding of visual encoding because if stimulus-specific adaptation can be demonstrated, then it can be inferred that the given attribute is explicitly coded by the visual system.

The process of adaptation also maximizes information transmission. An optimal information transfer system has to strike a compromise between removing redundancy from the information it receives to increase efficiency and maintaining enough redundancy so that information is not lost by the inherently 'noisy' encoding processes (Barlow, 1989; but c.f. Barlow 2001). The Bayesian approach has been shown to provide a method by which humans can combine prior knowledge of the environment and 'noisy' sensory input (Barlow, 2001; Berkes et al., 2011; Clifford et al., 2007). This framework can also account for adaptation effects by assuming a shift in the likelihood function after adaptation increasing the signal to noise ratio (SNR) of stimulus values in the vicinity of the adaptor (Clifford et al., 2007). Adaptation adjusts the gain of the response around the adapting value, narrowing the conditional probability density in the vicinity of the adaptor and increasing the reliability of the measurement (Stocker \& Simoncelli, 2006). 
This increase in gain around the adaptor explains decreases in discrimination thresholds seen in adaptation paradigms because the narrowing of the conditional probability density around the adaptor effectively increases sensitivity in that region (i.e. the likelihood is increased near the adapted orientation; see Stocker \& Simoncelli, 2006). The shift in likelihood function can also explain the repulsive effects of adaptation because by increasing the SNR in the vicinity of the adaptor (narrowing the conditional probability density), the SNR away from the adaptor is necessarily decreased (broadening the conditional probability density). This asymmetric change in likelihood pushes the mean away from the adaptor value causing the repulsive effects commonly seen in adaptation paradigms (Stocker \& Simoncelli, 2006). As mentioned above, an optimal information transfer system has to strike a compromise between removing redundancy (efficient coding) and adding redundancy (increasing the signal to noise ratio). Adaptation changes the intrinsic signal response probability (likelihood) to more accurately match that of the adapting stimulus thereby satisfying the latter of these constraints (Wainwright, 1999).

As described above, adaptation can also be viewed as a gain-control process. At low levels of the visual hierarchy, retinal ganglion cells can only code a 1-2 log unit change of light level in their firing rate, yet the eye functions over 10 log units by continuously resetting (adapting) retinal sensitivity to match current luminance levels (Ohzawa, Scalr, \& Freeman, 1985). At higher levels, neurons adjust their contrast response functions (laterally shifting the response and/or decreasing the slope) to match the mean level present in the current stimulus via contrast gain control. That is, adaptation is also the process that allows us to perceive and discriminate contrast differences across the highly varied visual situations that we encounter in our visual 
environment (Ohzawa, Scalr, \& Freeman, 1985). Some have theorized that adaptation in early levels of visual encoding (such as the retina) serves to maximize information transmission by optimizing spike times (Brenner et al., 2000). However, spiking rates cannot explain the effects of adaptation at higher levels of processing in which it is assumed that a linear-nonlinear filtering mechanism accounts for adaptation effects; here it is the filtering properties that must change in response to stimulus statistics (Clifford et al., 2007). The non-linear filtering mechanism must adapt its processing to account for the recent stimulus input and adjust according to the gain imposed by adaptation. However, both levels of change induced by adaptation can be thought of as a change in the gain of either the single cell or the filtering process. Moreover, this similarity can be expanded to include the effects of masking as a kind of very short-term adaptation response. Contrast adaptation can be modeled in a very similar manner as masking in that adaptation would be a form of gain control that is conducted across time while masking gain control would be conducted locally in time (Abbonizo, Langley, \& Clifford, 2002; Foley, 1994; Greenlee \& Heitger, 1988). Although adaptation effects and masking effects may have different mechanisms of implementation, the important comparison is that they can both be modeled in the same way. Adaptation, like masking, would simply be another way of normalizing the current response and changing the likelihood distribution.

Although there are many models of adaptation, there are two predominant camps: the multiple-channel model and the normalization model (Webster, 2011). The multiplechannel model is probably most familiar: narrowly tuned channels respond to a specific stimulus and the suppression of one channel leads to adaptation in the response. 
Prolonged stimulation to a certain adapting stimulus causes the channel most closely tuned to that adaptor to be suppressed. The overall pattern of activity (across all channels) is then changed due to the suppressed response from the adapted channel (Webster, 2011). One of the most cited examples of this type of adaptation would be orientation aftereffects wherein adapting to a grating of one particular orientation affects sensitivity to gratings of that and surrounding orientations (Blakemore \& Campbell, 1969; Gilinsky, 1968; Regan \& Beverly, 1985). The multiple-channel model predicts the repulsive effects of adaptation such as when stimulus levels just above or below the adapting value are biased away from the adapting value. In contrast, the normalization model proposes a more norm-based coding of stimulus inputs; that is, stimuli are encoded relative to (as deviations from) the norm or base level. These types of adaptation occur when prolonged exposure to a stimulus causes a renormalization of perception such that the adapting stimulus appears more neutral; for example, when colors fade to grey as one continues to stare at them (Webster, 2011). In this case, either the channels coding the input are broad or the stimulus itself is broad in nature: color adaptation normalizes the response across three broadly tuned cones. Similarly, adapting to broadband, natural stimuli would cause changes across a wide range of narrowly tuned spatial frequency filters (Webster, 2011). Either of these two cases leads to a re-normalizing of the overall response. These two models are not necessarily mutually exclusive and very rarely do we adapt to a narrow-band stimulus in our natural world. Therefore, it is important to remember that different modalities may have different ways of encoding information, but may still act under the same type of adaptation process: that is, they are still attempting to normalize responses. 


\section{Adaptation: Key Sensory Changes}

Adaptation experiments have proved to be very important in discovering many of the processes underlying encoding of sensory information. The relevant key findings will be summarized here. Stimulus-specific adaptation has shown us that the processing of spatial frequency, for example, is coded by multiple underlying channels. Adapting to a specific spatial frequency causes a decrease in sensitivity only over a small range of spatial frequencies rather than changing the contrast sensitivity of the entire system indicating that spatial frequency is encoded via multiple, narrowly tuned channels (Blakemore \& Campbell, 1969). Similarly, adaptation studies have also demonstrated that stimulus orientation is encoded by units tuned to different orientations. After adapting to a vertically oriented grating, subjects report seeing other, near vertical gratings as tilted away from vertical and acuity for vertical gratings is decreased (Gilinsky, 1968). It is concluded that the perception of orientation and spatial frequency is determined by 'channels' tuned to a specific range of orientations or spatial frequencies with specific tuning bandwidths around the center value.

The visual system adapts not only to simple aspects of a stimulus (single contrasts, spatial frequencies, orientations, etc.), but also to more complex environmental content. As mentioned above, the natural environment consists of content at multiple spatial frequencies and orientations and this content tends to exist in regular and predictable statistical patterns. If adaptation truly is a process which serves to optimize information transmission, it should optimize the response across more complex stimulus characteristics as well (e.g. first, second, and even third order image statistics). Human subjects have shown traditional adaptation effects (i.e., a shift in discrimination 
thresholds) for many complex stimuli properties including the appearance of facial features, depth and size of rooms, type of outdoor scene and other complex image properties (Kelly, Donaldson, Sjolund, \& Freiberg, 2013; Leopold, Rhodes, Muller, \& Jeffery, 2005; Muller, Metha, Krauskopf, \& Lennie, 1999; Webster, 2011; Webster, Georgeson, \& Webster, 2002; Ziemer, Plumert, Cremer, \& Kearney, 2007). For example, subjects have also been shown to adapt to the level of blur or sharpness (an image property inherently tied to slope of the amplitude spectrum) present in images: when adapted to blurred images, a previously in-focus image seems too sharp and vice versa (Sawides, de Gracia, Dorronsoro, Webster, \& Marcos 2011; Webster, Georgeson, \& Webster, 2002). Similarly, Webster and colleagues (1997) examined how the spatial structure of natural images might lead to continual states of spatial contrast adaptation. They hypothesize that the $1 / \mathrm{f}^{\alpha}$ amplitude spectrum slope relationship in natural images may ubiquitously adapt people so that they have lowered sensitivity to low spatial frequency information -- the preponderance of low spatial frequencies in natural images reduced observers' sensitivity to these spatial frequencies just as would have been expected in stimulus-specific adaptation (Haun \& Essock, 2010; Webster \& Miyahara, 1997). This continuous adaptation to the content of natural scenes would then serve to determine the 'natural operating state' of our visual system.

\section{Recent Relevant Findings}

Although many have speculated as to the functional benefit of adaptive processes (i.e., improving discrimination thresholds for certain stimulus parameters), it remains unclear exactly why the relationship between environmental statistics and visual encoding exists. Many have hypothesized that the efficient coding processes involved in 
the horizontal effect and $1 / f$ sensitivity may be inherent in our visual brain; since we evolved in a world that is biased towards low spatial frequencies and horizontal orientations, our visual system should have evolved to process these biases efficiently (Bex, Solomon, \& Dakin, 2009; Brenner, et al., 2000; Hansen \& Essock, 2004; Webster \& Miyahara, 1997). Some believed, then, that the bias present in cortex was at least somewhat hard-wired, present from infancy and continuing throughout development. However, recent converging evidence suggests that the brain may be even more efficient than previously thought, using adaptation techniques to acclimate to the statistics of the current/recent environment, whatever they may be. Very recent findings in the development of complex visual processing indicate that the efficient processing of visual statistics does not develop until around 10 years of age (Ellemberg, Hansen, \& Johnson, 2012; Ellemberg et al., 2012; Pei, Baldassi, \& Norcia, 2012). Additionally, recent reports exploring complex adaptation paradigms have discovered that the process of adaptation itself is not as clear cut as once thought (Chopin \& Mamassian, 2012). These findings combined with evidence from other perceptual learning studies have led to the conclusion that the relationship between the statistics of the natural world and visual processing could be a result of recent exposure via adaptation processes (Essock, Haun, \& Kim, 2009; Schweinhart \& Essock, 2013).

If the reciprocal relationships between the orientation/spatial frequency content of natural scenes and the encoding of this content described above were based on an evolutionary time scale, one would think that they would perhaps be present at birth, be 'hard-wired' (non-changeable), and be present regardless of visual experience. Given the above information regarding the presence of the oblique effect in infancy, it could be 
hypothesized that the horizontal effect may be innate if it is based on the number or response of cells tuned to cardinal orientations. On the contrary, converging evidence is showing that the 'anti-correlation' between natural scene statistics and visual processing biases shown in adult orientation and spatial frequency processing does not develop until $\sim 10$ years of age. As mentioned, human discrimination of images is optimal when the slope of the amplitude spectrum matches that found in typical natural scenes (Bex, Solomon, \& Dakin, 2009; Field \& Brady, 1997; Hansen \& Hess, 2006; Johnson et al.,2011; Parraga, Troscianko, \& Tolhurst, 2000; Tolhurst, Tadmor, \& Chao, 1992). Ellemberg, Hansen, and Johnson (2012) recently conducted the first study testing young children on a slope discrimination task wherein subjects are asked to distinguish between two simultaneously presented stimuli with different slopes (alpha) of the amplitude spectrum. Subjects were presented with images of natural scenes that had been manipulated to have amplitude spectra with different slope values $\left(1 / \mathrm{f}^{\alpha}\right.$ where $\alpha$ took values from -.7 to -1.3 but is normally -1 ). Subjects had to indicate whether two simultaneously presented images with different slope values were the same or different (Ellemberg, Hansen, \& Johnson, 2012). Typically, such alpha discrimination tasks show improved discrimination thresholds when alpha is equal to -1 , as it is in natural images (Hansen \& Hess, 2006; Knill, Field, \& Kersten 1990; Tadmor \& Tolhurst, 1994). Interestingly, these researchers found that the improved discrimination thresholds for stimuli which have near-natural slopes found in adults were present in 10 year olds, but absent in 6 and 8 year old children.

Similarly, Ellemberg and colleagues (2012) measured orientation discrimination thresholds for spatially 'noisy' stimuli in children and adults. Orientation discrimination 
for such stimuli is also not adult-like until 10 years of age; specifically, the introduction of oriented noise increases threshold for all groups, but significantly more so for younger children (Ellemberg et al., 2012). Thus, the particular sensitivity to the spatial frequency regularities of natural scenes as well as orientation discrimination abilities seen in adults develops slowly over the course of childhood rather than being present at birth. In a third line of evidence, VEPs were recorded from children and adults undergoing a visual masking paradigm presented at different levels of contrast (Pei, Baldassi, \& Norcia, 2012). Unmasked contrast response thresholds were largely similar across children and adults, however, masking impacted children's response functions differently; in children, the slope of the response function changed rather than a lateral shift of the function as is seen in adults. The authors concluded that contrast normalization processes are not fully formed in 'school-aged' children (Pei, Baldassi, \& Norcia, 2012). It is clear, then, that the proposed efficient processing of statistical regularities is not, in fact, present at birth as it requires time to develop, perhaps because experience with the natural scene statistics are required before a representation of a prior probability distribution can be established. The bias in visual processing could still be innately determined, however, simply requiring a longer time scale to develop than previously thought. One key to determining the difference will be examining whether or not it takes experience with the natural world to develop the efficient coding relationships but another, and the one explored here, will be determining whether deviant experience can change the visual biases.

\section{Perceptual Learning}

The above mentioned theories postulate that the visual system uses adaptation to calibrate itself to the current environmental statistics in order to effectively process 
information (Webster, 2011). This idea is not all that surprising when one takes into account the large amount of literature on perceptual learning. This literature has shown that perceptual biases can change based on training or experience, but that this training is not necessarily permanent. For example, recent work into multisensory binding has shown that the temporal window of binding can be widened or narrowed via perceptual experience with different temporal displacements (Powers, Hillock, \& Wallace, 2009). Moreover, this perceptual learning is susceptible to reinforcement such that reward affects the strength of learning (Marx \& Einhauser, 2015). In fact, even the oblique effect can be changed via perceptual learning when observers are trained to discriminate oblique orientations (Mayer, 1983; Song et al., 2010; Vogels, \& Orban, 1985). Starting with subjects who demonstrated a traditional oblique effect (lower sensitivity to oblique orientations) researchers were able to improve detection thresholds through practice at detecting oblique orientations. By the end of 3000 practice detection thresholds, all observers had improved their sensitivity to oblique orientations and most showed similar sensitivity to cardinal and oblique orientations (Mayer, 1983). Practice with cardinal orientations did not improve thresholds unless the subject already had elevated thresholds to cardinal orientations (Mayer, 1983). Moreover, practice discriminating one oblique orientation (45) can improve thresholds for another oblique orientation but not for cardinal orientations meridians (Westheimer \& Lavian, 2013). (This transfer of training effect could be due to the class II oblique effect as the oblique orientations are often confused in memory.)

The neural mechanisms presumed responsible for the oblique effect also change after perceptual training. Before training, subjects showed an oblique effect pattern of 
lower detection thresholds for horizontal than oblique orientations coupled with higher fMRI responsiveness to the cardinal compared to oblique orientation (Furmanski, Schluppeck, \& Engel, 2004). After more than 20 daily training sessions with the oblique orientation, subjects showed improved detection of the oblique grating and increased responsiveness of V1 to the practiced oblique orientation, but not to the unpracticed orientation (Furmanski, Schluppeck, \& Engel, 2004). Similarly, perceptual learning on an orientation discrimination task also increases the neural response; observers trained to discriminate small changes in orientation showed an enhanced neural representation of the trained orientations (Jehee, Ling, Swisher, van Bergen, \& Tong, 2012). It can therefore be concluded that, although perceptual learning (like masking) research has been separated from adaptation literature in the past, there may be no reason to assume that the two are in fact functionally different when one takes a Bayesian approach. Both are simply cases of new experience altering the current perceptual bias, and perceptual learning paradigms include a training and feedback component. Perceptual learning general takes a longer amount of time, but in the above cases especially, there is no reason to assume that it is operating under a different frame-work than that used for adaptation.

\section{Background of the Current Project}

The primary impetus for the current project comes from relatively recent work showing that perceptual biases can be altered simply by experiencing a different bias in the natural environment (Bao \& Engel, 2012; Kwon et al., 2009; Zhang et al., 2009). This work has called into question the idea that our brain is hard-wired to encode the typical (average) content we (i.e., our evolutionary forbearers) encountered in our 
environment. Firstly, researchers found changes in contrast sensitivity and fMRI BOLD responses in early visual areas after prolonged monocular adaptation to a contrast reduced environment (Kwon et al., 2009). Subjects wore contrast-reducing goggles for four hours that caused subsequent decreases in thresholds as well as increases in the BOLD signal in $\mathrm{V} 1$ and V2. The results transferred to un-adapted eyes indicating that the shift in contrast gain was cortical in nature (Kwon et al., 2009). The authors distinguish between contrast gain and response gain models of adaptation indicating that the longer-term adaptation paradigms (such as used here) involve changing the range of the response (response gain) rather than simply shifting the slope (contrast gain).

Secondly, in a pair of studies Engel and colleagues developed a method for altering the orientation content in the visual environment by mounting a digital video camera to a portable head mounted display (HMD). The camera then connected to a laptop that was able to process the image captured by the camera (640x480 pixels at 8 bits presented at 30 frames per second ) and filter it in near-real time to project it in the HMD (field of view of $37.8 \times 30.2$ degrees) (Zhang et al., 2009).The filtering process involved conducting a fast Fourier transform of the image and convolving this with filters at different orientations to decrease/increase the visibility of these orientations in the resulting image (decreasing/increasing the relative amplitude of these specific orientations). The filters used in the Fourier domain were centered at the specified orientation with a Gaussian profile that removed energy at the specified orientations (standard deviation of $30^{\circ}$ : $\mathrm{FWHH} \approx 70^{\circ}$ ) across all spatial frequencies (Zhang et al., 2009). The filters used completely removed the energy at the specified orientation equally across the two studies. Observers were then able to interact with an environment 
with altered orientation content via the HMD. Observers wore the device for four hours during which they were deprived of either horizontal or vertical orientation information via the filtering process. During adaptation subjects performed normal activities (e.g., walked around, watched movies, and ate).

After adaptation with the HMD, contrast detection thresholds for sinusoidal gratings at the deprived orientation were significantly lowered; adaptation to an environment lacking these orientations increased the observers' sensitivity to them (Zhang et al., 2009). However, this increased sensitivity was lost throughout the testing phase of the experiment presumably because it was short-term or because the subjects were exposed to the previously deprived orientation. In the first half of post-adaptation testing (the first three staircases at each testing orientation: 20 minutes), subjects showed a $15 \%$ decrease in detection thresholds for the deprived orientation (as compared to a control group), while in the second half of testing (the last three staircases: last 20 minutes) the difference was not significant. By the next day of testing, thresholds had fully returned to baseline, although subjects were not tested other than 24 hours later (Zhang et al., 2009). Moreover, narrowing the filter parameters both in terms of spatial frequency and orientation (2/3-4 CPD and $\left.70-110^{\circ}\right)$ did not significantly change the magnitude of the decrease in detection thresholds post-adaptation (Zhang et al., 2009). Although this paper did not include the post-adaptation time intervals (only first 20 and last 20 minutes) or the number of trials, one can conclude that the duration of adaptation effects is rather short as they only occurred in the first half of post-adaptation testing.

In a second paper, Bao and Engel (2012) used the same method to test how long the effects of adaptation lasted using different adapting times (1, 4, or 8 hours). This 
paper sought to determine whether the length of adaptation time effects both the duration and strength of adaptation, as well as investigate the mechanisms behind short and long adaptation effects. The short-term adaptation effects were tested by 'de-adapting' observers after the long-term adaptation by showing them images of natural scenes. The task involved the perception of line orientation as measured by the tilt after effect (TAE). Subjects were presented with plaid patches made up of two sine-wave gratings which resembled a blurred checkerboard pattern. In a typical adaptation experiment, the component sine-waves of the plaid appear tilted away from the adapted orientation making the checks of the plaid appear rectangular. The subject's task is to tilt the component gratings of the plaid until the checks appear square. For this study, a positive effect indicates that the subjects shifted the component sine-waves towards vertical as the plaid was diagonally oriented. Subjects tested in all conditions showed a positive TAE in response to adaptation with attenuated vertical content indicating increased gain at vertical orientations in the population of cells which respond to vertical orientations (Bao \& Engel, 2012). Longer adaptation times produced longer-lasting as well as stronger effects of adaptation; adapting subjects for 1 hour showed effects only for the first 1-2 minutes of post-adaptation testing, while adapting for 4-8 hours showed effects lasting beyond the post-testing period (6 min) (Bao \& Engel, 2012).

Bao and Engel (2012) went on to test the short- versus long-term adaptation using a traditional learning paradigm. After adapting subjects over 4 hours, they immediately de-adapted them for 15 minutes by showing them images of natural scenes. Subjects initially showed no effects of adaptation: just as an animal may be taught to associate one behavior with a certain stimulus and then taught a new behavior which overrides the 
older conditioning. However, just as in classical conditioning, subjects showed 'spontaneous recovery' of the adaptation effects after continued testing. The authors suggested that the de-adaptation and subsequent recovery was evidence for two adaptation mechanisms: a long-term mechanism more affected by longer experience and a short-term mechanism malleable by more recent experience. These two controlling mechanisms increase (attenuated content) or decrease (enhanced content) the gain of cells according to the presence of stimuli near their peak tuning preference in the recent environment. The long-term mechanism needs hours of experience to adapt and its effects last much longer whereas the short-term mechanism can be adapted in as little as a few minutes and decays equally as quickly. Fitting with these findings, Chopin and Mamassian (2012) have recently discovered that there may be more of a hierarchical relationship between adapting to recent stimuli and more remote experience. In their study, the authors showed in two experiments that it is the remote past which the visual system relies on to estimate the world's orientation distribution. The percept that results from adaption can be predicted from assuming that it will be that percept which helps the most recent experience match that of the more remote past. In other words, observers' perception is biased not only by the recent environmental exposure, but also by a more long-term experience with their environment; the recent past can change perceptual biases, but only on a limited basis.

In the studies discussed above, subjects experienced the environment in real time while getting 'feedback' from interacting with it (i.e., walking around, picking up objects etc). Is this type of immersive experience necessary? As previous work has indicated that adaptation to static, passively viewed stimuli may be enough to induce perceptual 
changes, one might conclude that interaction with the environment is not necessary to induce effects (Webster, Georgeson, \& Webster, 2002; Webster \& Miyahara, 1997). However, literature from virtual reality has suggested that immersive experience with objects improves encoding in comparison to passive observation, suggesting that immersive experience may lead to stronger effects (James, Humphrey, \& Goodale, 2001; James et al., 2002). When participants are allowed to guide the visual study of an object, the objects are recognized faster than when participants have the same passive views of the same objects (James et al., 2002). Such 'active' viewing also leads to faster performance on a mental rotation task involving the studied object (James, Humphrey, \& Goodale, 2001). Moreover, adults learn novel visuo-auditory associations more efficiently when allowed to haptically interact with visual stimuli than when they passively view such stimuli, perhaps due to increased gain in sensory motor area STS following active motor learning (Butler, James, \& James, 2011; Fredembach, de Bolsferon, \& Gentaz, 2009).

In contrast to adaptation paradigms, all of these active vs. passive learning studies used the same stimuli in the viewing/learning phase of the experiment. Although interactivity may simply improve performance due to an increase in visual input in the encoding phase, it is true that immersive, interactive experience leads to more efficient visual encoding (Keehner, Hegarty, Cohen, Khooshabeh, \& Montello, 2008). Thus far, adaptation studies have only indicated whether the experience was immersive (Bao \& Engel, 2012; Zhang et al., 2009) or passive (Webster \& Miyahara, 1997), but not tested the magnitude of the adaptation effect in both immersive and passive conditions to allow direct comparison. The type of experience (immersive or passive) with the environment 
may serve to strengthen adaptation effects acting as a feedback/reinforcement altering perception to a new and different environment (Butler, James, \& James, 2011;

Fredembach et al., 2009; James, Humphrey, \& Goodale, 2001; James et al., 2002; Marx \& Einhauser, 2015).

As discussed above, orientation processing, like many other aspects of the visual (and perhaps all sensory) system is biased and has some relationship to the orientation content present in natural scenes. Previously, we thought that this relationship may have been hard-wired in cortex due to presenting a biased environment to the visual system across millennia. Alternatively, some have theorized that we are 'constantly adapted' to environmental statistics and that this should be taken into account when testing subjects using unnatural stimuli (Webster \& Miyahara, 1997). No one to date has studied how adapting to different environmental conditions affects the horizontal effect of orientation processing. The recent work by Engel and colleagues $(2009 ; 2012)$ has shown that the horizontal effect should be alterable given different experience with an 'unnatural' world. Through the use of adaptation paradigms this work demonstrates that one can alter the horizontal effect simply by changing an observer's environment. This study shows that the horizontal effect is a result of the observer's exposure to environmental statistics and that experience with different statistics changes the perceptual biases of the effect. 


\section{GOALS}

The primary purpose of this study is to determine if experience with altered environmental statistics modifies the anisotropic suppression of the horizontal effect. Presumably, if the horizontal effect is influenced by the orientation distribution in the environment, experience with an altered visual environment will change this perceptual bias. The studies conducted by Engel and colleagues $(2009,2012)$ showed modified narrowband orientation processing after a few hours of exposure with an altered environment. The experiments described below were designed to determine if the horizontal effect, assumed to be caused by differential suppressive weights of the filters involved in visual orientation perception, can be modified in a similar amount of time. Previous adaptation in an immersive environment showed that increasing the time spent adapting increases both the magnitude and duration of narrowband, post-adaptation perceptual change (Bao \& Engel, 2012; Zhang et al., 2009). Experiment 1 sought to determine whether different durations of experience in a similarly altered environment alter perception across different orientations in broadband scenarios.

Previous work has also indicated that changing the center orientation and spatial frequency bandwidth of the image processing filter had little effect on the amount of perceptual changed induced by adaptation (Zhang et al., 2009). That is, adaptation to horizontal or vertical deprivation or enhancement across multiple spatial frequency bandwidths all induced similar types and strengths of perceptual change. However, to 
date, no one has examined the relationship between the magnitude of physical change that the filter induces and the magnitude of the perceptual change. Therefore, Experiment 2 was designed to test whether varying the amount of physical change in the environment affects the strength of the perceptual change after adaptation. As stated above, no direct comparison has been made between static/passive adaptation and immersive adaptation. To investigate this, Experiment 3 included two kinds of passive viewing conditions in which observers simply viewed filtered videos of the environment to determine the extent to which immersive experience in an altered environment is required to enact perceptual change. The first three experiments served as a systematic investigation into how adaptation in an altered environment affects broadband orientation perception. Lastly, and crucially, Experiment 4 examined whether experience in an isotropic environment altered or eliminated the perceptual biases of the horizontal effect. Since the isotropic condition will be the basis for the model, two other conditions were also run as compliments to changing the entire distribution of orientations: a 'enhancednatural' condition in which the natural cardinal/oblique anisotropy is exaggerated and an 'oblique dominant' condition in which the typical cardinal supremacy is flipped so that the distribution peaks at the obliques and exhibits minima at the cardinals. 


\section{METHODS: Testing of Performance at Different Orientations}

In order to determine the effect that adapting to different environmental statistics has on orientation perception, observers' bias across orientations before and after adaptation (i.e., the horizontal effect) was measured using a suprathreshold matching paradigm. This type of broadband matching experiment has been shown in the past to evoke the horizontal effect; subjects need 'more' content (higher amplitude) at horizontal test orientations than at the obliques to perceptually match a standard pattern (Hansen \& Essock 2004, 2006). Before and after adaptation, subjects completed a matching task in which the perceived orientation content of a test pattern was adjusted to match that of a standard. Both test and standard stimuli consisted of a $1 / f$ noise pattern which had been convolved in the frequency domain with an oriented filter designed to increment one band of orientations above the background noise (see Figure 1). As previous adaptation effects were only measured for 2-6 minutes after adaptation (Bao \& Engel, 2012; Zhang et al., 2009), the time it took subjects to complete each match was recorded so that adaptation effects could be tracked over the course of the testing period.

\section{Methods: Stimulus Creation and Procedure}

Stimuli consisted of circular patches ( 512 x 512 pixels in diameter) of broadband $1 / f$ noise patterns with an incremented orientation component (Figure 2). To create these stimuli, the spatially broadband, isotropic amplitude spectrum of a randomly generated noise pattern was convolved with a spatially broad, triangular filter. The shape of the 
filter was such that it peaked at the test orientation and linearly declined to 0 on either side of a $20^{\circ}$ wide orientation bandwidth (see Figure 1). These filters increment content

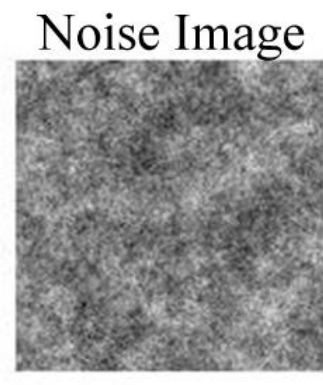

Filter 1.3

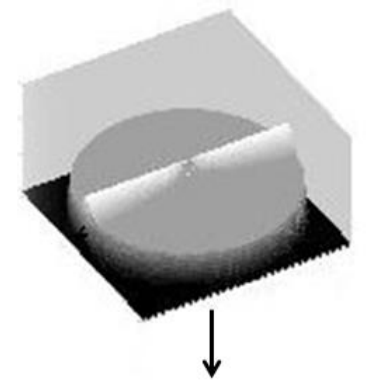

1.3 Image

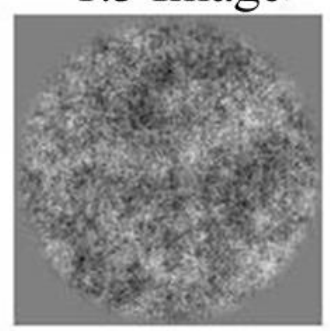

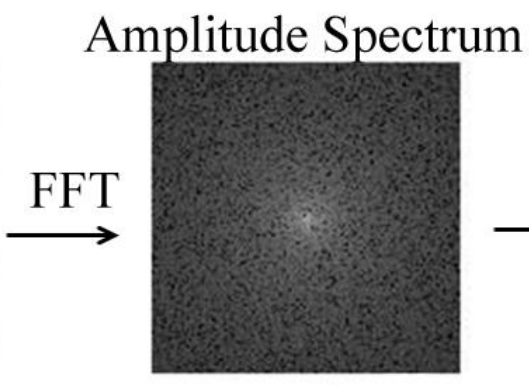

Filter 1.6 (standard)
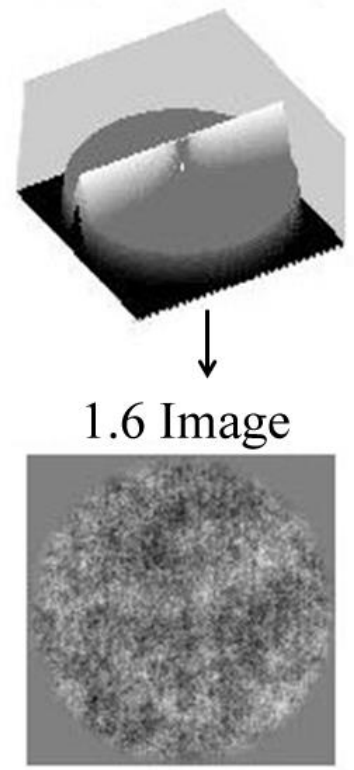

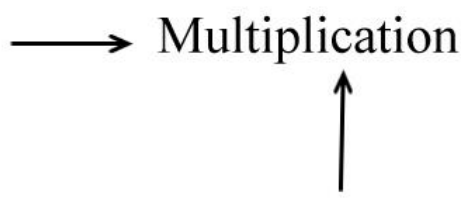

Filter 1.9
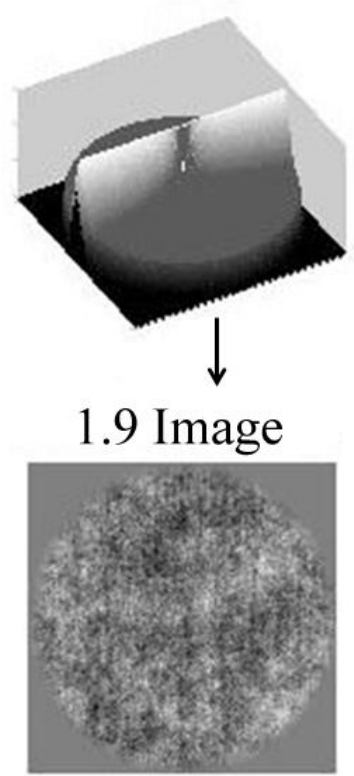

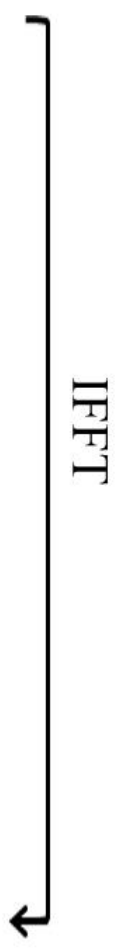

Figure 1. Creation of incremented, spatially broadband, oriented noise stimuli.
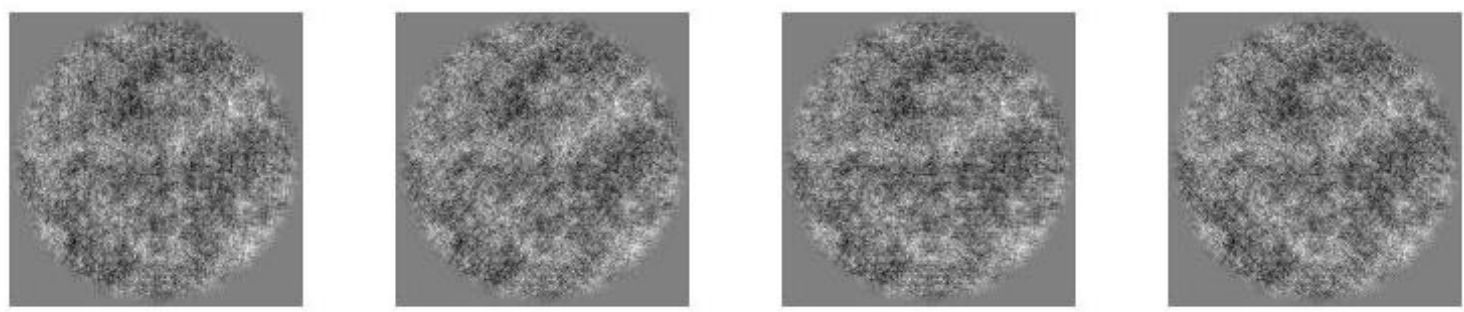

Figure 2. Example stimuli used in the matching task from left to right: vertical, forty five degrees, horizontal, and one thirty five degrees. All orientations are presented with the same orientation increment (1.6). 
within the orientation band of interest above the background of isotropic noise. For the standard stimuli, the orientation filter was 1.6 times the amplitude of the background noise. The increment of the test stimulus was randomly set to be between 1.3 and 1.9 times the background noise at the beginning of each trial and then manipulated by the subject to perceptually match the standard; thus, some trials began with the test stimulus having a lower increment than the standard and some started with a higher increment. Subjects adjusted the increment of the test stimulus to match the perceived increment in the standard using both large $( \pm .5)$ and small $( \pm .2)$ increments and decrements via key press. The center orientation of the test stimulus was block-randomized so that subjects viewed each test orientation every 4 trials. The magnitude of the test increment and phase spectrum of the background noise was determined randomly on each successive trial (phase spectrum of the test and standard matched on every trial).

Subjects viewed a $22.5^{\circ}$ oriented standard stimulus with a fixed increment magnitude (1.6 times the background) on the right and a variably oriented $\left(0^{\circ}, 45^{\circ}, 90^{\circ}\right.$, $\left.135^{\circ}\right)$ and incremented (1.3-1.9 times the background) test stimulus on the left. Standard stimuli were presented on a Nanao Flexscann F2-21 20" CRT monitor and test stimuli were presented on a Samsung Syncmaster 1100 20" CRT monitor. Monitors were calibrated to express linear luminance values and match each other $\left(r^{2}=.998\right)$. Standard stimuli were created with an orientation increment at vertical on the monitor but the monitor itself had been rotated (and fixed) to a $22.5^{\circ}$ position. This physical rotation was performed to better equate pixel characteristics at this orientation with those of the test stimuli making the matching task easier (see Hansen \& Essock 2004 for discussion). The two stimuli were each $6.25^{\circ}$ in diameter and viewed through masks that occluded monitor 
bezels and other room structure. The standard and test stimuli were presented at eye level centered $16.9^{\circ}$ apart and viewed in a dark room. Subjects were positioned by chin/forehead rest at $2.37 \mathrm{M}$. Every pre-test consisted of 36 trials (9/test orientation) with each test orientation $\left(0^{\circ}, 45^{\circ}, 90^{\circ}, 135^{\circ}\right)$ randomly presented within test blocks. The posttest was run for 30 minutes immediately following adaptation (unless otherwise noted) and subjects were instructed to complete as many accurate matches as possible in the 30 minute time frame. Most subjects completed between 70 and 120 total trials within the 30 minutes. As in the pre-test, test orientations were randomly presented within test blocks and the time was recorded for each trial. Keeping the post-test time fixed ensured that all subjects were exposed to normal visual stimuli for the same amount of time postadaptation, while allowing them to complete as many trials as possible (i.e., without limiting data collection).

The subject's task was to change the magnitude of the increment applied to the test pattern to match the perceived amplitude of the standard pattern. The ratio of the test orientation's increment needed for the observer to perceptually match the $22.5^{\circ}$ standard's increment and the value of the fixed increment in the standard was obtained over repeated trials and averaged. (Ratios less than 1 indicated that the observer saw that test orientation better than the standard, needing less of a physical increment to match the salience of the standard stimulus, and ratios greater than 1 indicated that the test orientation was seen less well than the standard.) The mean and standard deviation of the observers' match values were taken to reflect the internal perceptual bias and perceptual variability, respectively (Girshick, Landy, \& Simoncelli, 2011). All subjects were prescreened to ensure normal visual acuity and that they showed a clear horizontal effect 
pattern in pre-testing, needing a larger test increment to match horizontal test patterns over oblique ones. The pre-screening also served as a training session on the psychophysical matching task.

\section{Methods: General Adaptation Paradigms and Analyses}

After completing the screening and the pre-test (36 trials, 9 trials at each test orientation), subjects adapted to an altered environment for a pre-determined amount of time (varied in Experiment 1, otherwise 2 hours). Unless otherwise noted (i.e.

Experiment 3), during adaptation subjects wore a HMD (Sony Personal 3D Viewer) with an attached lightweight, black and white digital camera (NET CMOS iCube USB 3.0 camera). The current visual environment that a subject was experiencing was acquired through the camera and sent to a laptop (HP ENVY 15t-j100) which filtered the camera images frame by frame and fed the filtered images to the HMD to be viewed in near realtime by the subjects as they interacted with the environment (Figure 3). All filtering of the environment took place in Matlab (MATLAB 8.2, The MathWorks Inc., Natick, MA, 2013) using the image acquisition, signal processing, and image processing toolboxes. Each frame of the video output was fast Fourier transformed (FFT) and the resulting amplitude spectrum was altered to attenuate (or enhance) specific orientations (see Figure 4 for example). The root mean squared (RMS) contrast of the original image was imposed upon each altered image during the inverse Fourier transform thus ensuring contrast equality between frames. The exact filter parameters used varied in each experiment and are described below. The entire filtering process from image acquisition to presentation of the filtered image over the HMD took less than $35 \mathrm{msec}$, and, as was reported in previous studies, subjects were tolerant of this delay (Zhang et al., 2009). 
After adaptation, subjects completed as many matching trials as possible during the 30 minute post-test. The ten subjects ( 5 female, 8 naive) were students at the University of Louisville. All gave informed consent as per the protocol approved by the University's IRB (see Appendix). All were pre-screened to ensure normal or correctedto-normal vision. One other subject was dropped from the study due to experiencing nausea from wearing the head mounted display. Six of the ten total subject participated in more than one of the experiments listed below. To avoid order effects, subjects participating in more than one condition/experiment were assigned to the conditions randomly.

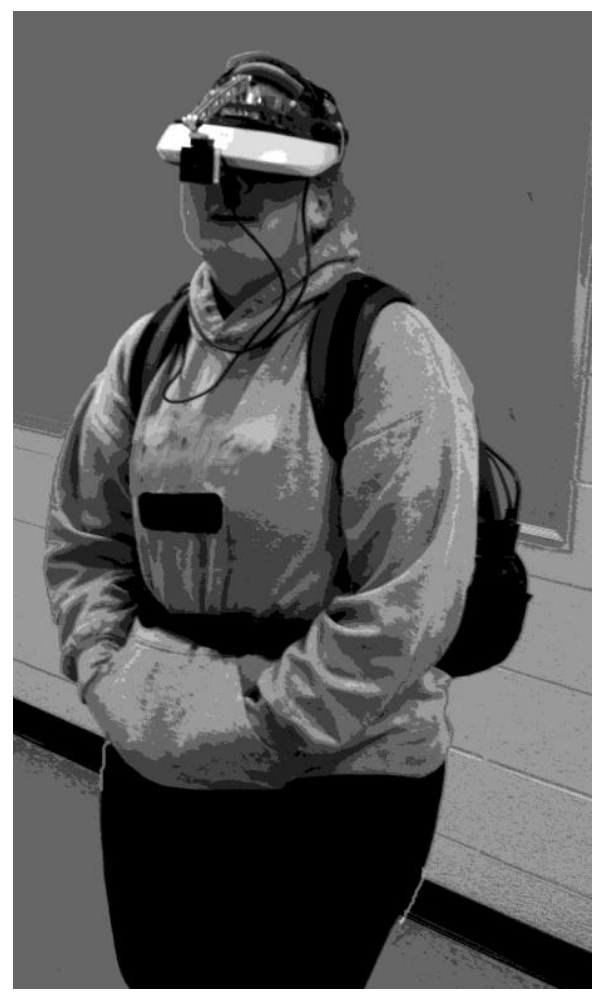

Figure 3. Example of a subject undergoing adaptation. The camera is situation in front of the visor of the HMD at the height of the subjects' eyes. The backpack the subject is wearing contains the laptop used for processing the images.

Unless otherwise noted, for every condition the pretest scores are computed from the average of each subject's pretest run (36 trials total) and the posttest scores are computed by averaging the first ten minutes of each subject's posttest period (i.e., the 
first third of testing). To quantify the results, the pre and post-test match values were subtracted (pre -post) to determine how adaptation in the altered environment affected perception of orientation in broadband stimuli. Additionally, a measure of the horizontal effect (horizontal match value minus the average of the two oblique match values) was computed before and after adaptation for each subject. Subtracting the pre and posttest horizontal effect scores allows one to see how the horizontal effect pattern changed as a result of experience in an altered environment. For the majority of experiments, the effect size (match value difference score/pooled standard deviation of match values: Cohen's $d$ ) was also computed to determine if the test manipulation affected the magnitude of the perceptual after effects.

\section{Methods: Video Analysis}

A digital recording of the environmental imagery that each subject was exposed to during adaptation was archived and used to characterize (unfiltered) typical visual experience for modeling purposes. The video was recorded directly from the camera feed and therefore consisted of an unaltered version of what the subjects viewed during adaptation. As the video recording lasted the entire adaptation period, they were prohibitively large for full analysis, so a sample consisting of one frame every 1000 frames was taken, FFT performed, and averaged. Each video recording was sampled and analyzed twice: once using the same filtering procedures on the recorded images that were used during adaptation and once without any alterations. The original, unaltered videos served as a 'typical' environmental distribution; that is, what subjects would have been exposed to under normal viewing conditions. As during adaptation, filtering of the video recordings involved the Image Acquisition Toolbox in Matlab. In short, a FFT was 
run on each frame of the video, the same manipulations used during adaptation were applied to alter specified orientations, images were RMS contrast corrected, the FFT process was inversed, and the orientation spectrum of the filtered frame was calculated using image rotation algorithms developed previously (Schweinhart \& Essock, 2013). This method accurately determines the amplitude of oriented content in an image every 3 degrees by physically rotating the image to the desired orientation and applying filters oriented at vertical and horizontal. The method avoids the inequalities in representing the oblique orientations inherent in running a discrete FFT (see Hansen \& Essock 2004, for discussion and Schweinhart \& Essock, 2013, for method).

Both altered and unaltered orientation spectra were transformed into probability distributions to compare the physical difference. The difference between these distributions was determined using Kullback-Leibler (KL) divergence. KL divergence is a measure of the information lost when when probability distribution is used to approximate another. The KL divergence scores from the filtered videos were then correlated with the raw difference scores (pre-post) in Experiments 2 and 4 to determine if the amount of physical change in the environment is directly related to the amount of perceptual change. The average altered orientation spectrum in each condition also served as the prior probability distribution after adaptation in the model discussed below. 


\section{EXPERIMENT 1: Build-up and decay of adaptation}

\section{Methods}

The first experiment tested the time-course of the build-up and decay of the perceptual effects of deprivation of specific orientations. Pilot testing confirmed the results of previous work: enhancement of orientations produced decreases in sensitivity while deprivation of specific orientations increased sensitivity at those orientations (Schweinhart, 2014; Zhang, et al., 2009). As the horizontal effect and its relationship to natural scene content is of particular interest here, the majority of experiments focus on the perceptual effect of horizontal deprivation. Previous work also indicated that subjects show some amount of altered orientation perception after one hour of adaptation in an altered environment, but that effects increase in strength and duration as the duration of adaptation period is increased (Bao \& Engel, 2012). Therefore, the focus of this experiment was to determine whether or not the positive relationship between perceptual effects and adaptation duration extends from a narrowband perceptual test to a broadband perceptual test.

To accomplish this, experienced psychophysical observers adapted to the same altered environment for variable amounts of time. Subjects wore the HMD and adapted to an environment in which horizontal content had been decreased by a fixed scalar for 5 , $15,30,45,60,120,180$, and 240 minutes. The filter used during adaptation consisted of the same type of triangular 'wedge' filter used in the psychophysical test. The adaptation 
filter attenuated horizontal orientations by $85 \%$ of their original value across all spatial frequencies within a $45^{\circ}$ bandwidth of orientations (see Essock et al., 2003; Hansen \& Essock, 2006; Figure 4). This type of triangular filter focuses the change specifically at the orientation of interest (the peak of the filter). The minimum of the filter (.15) is at horizontal and the attenuation is linearly ramped up from .15 to 1 across a bandwidth of $22.5^{\circ}$ on either side $\left(45^{\circ}\right.$ total bandwidth; Figure 4$)$.
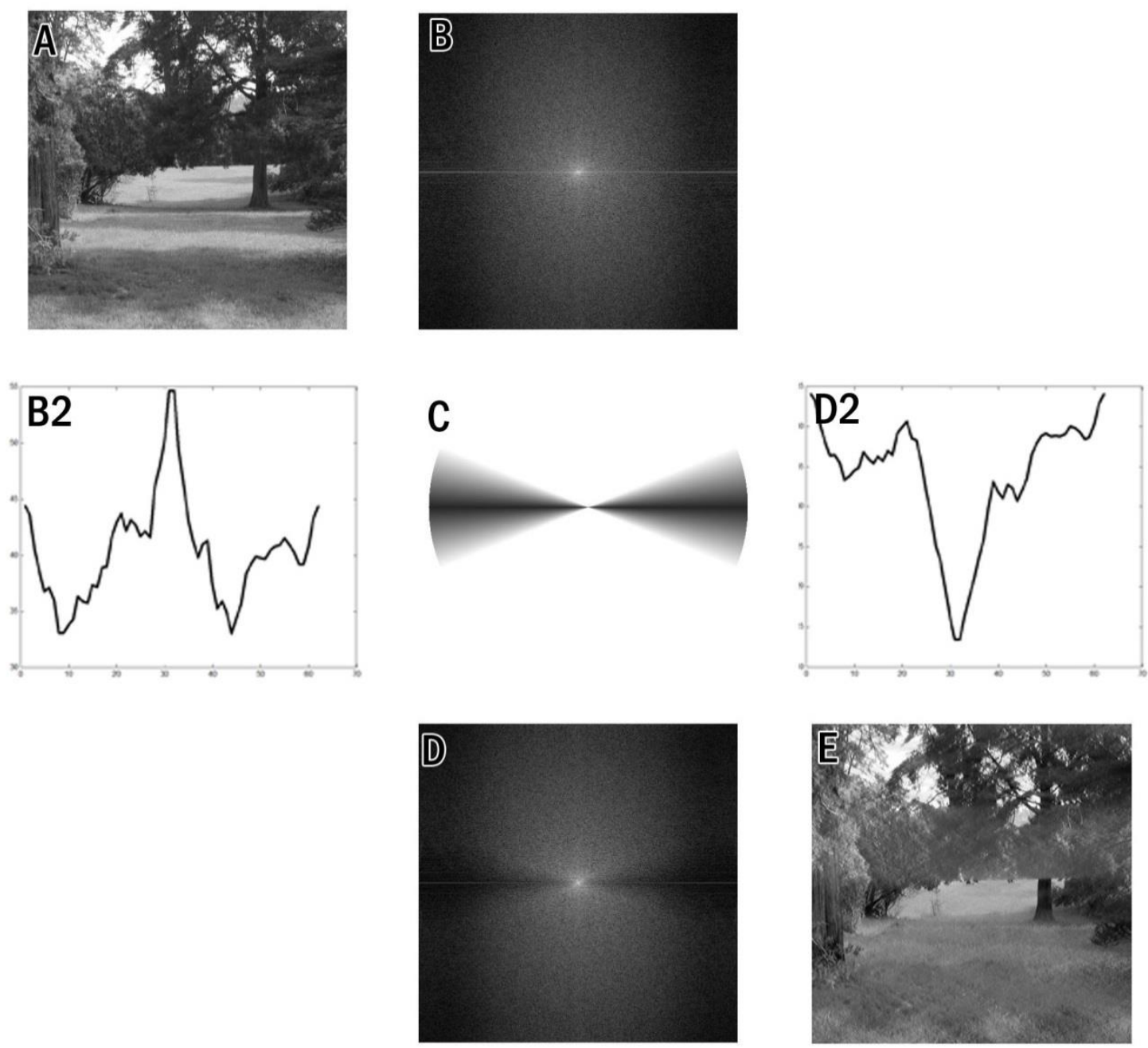

Figure 4. Steps in the filtering process used to decrement horizontal orientations (images spectra have been rotated to fit with space domain conventions). The original image (A) is fast Fourier transformed and the resulting amplitude spectra (B/B2) is convolved with a filter that decrements horizontal orientations (C) resulting in the altered spectra $(\mathrm{D} / \mathrm{D} 2)$ that is inverse transformed and presented to the viewer $(\mathrm{E})$. 
The specific conditions tested are listed in Table 1. A total of 3 subjects were assigned to run each duration condition in random order (S1, S2, and S5, all female, 2 naive). Data obtained during each trial block of the post-test of each of the duration conditions was also compared to determine the decay of adaptation effects. The time it took (minutes) for subjects' match values and horizontal effect score to return to baseline levels (within one standard deviation) across the duration conditions was computed and quantitatively compared using a floating average window towards the end of the post-test trials. Specifically, the match values at each test orientation as well as the horizontal effect score for each trial block were computed and averaged in windows of 3-trial blocks. If a subject's horizontal match value or effect score did not return to within one standard deviation of baseline levels, it was considered to take longer than 30 minutes for the subject's perception of horizontal orientations to return to normal. In calculating average decay rates, these cases were given a value of 30 .

\section{Results}

A one-way ANOVA was run on the horizontal match value difference scores, but was underpowered, $F(7,14)=1.64, p=.20$. The average measures of horizontal perceptual change are listed in Table land Figure 5.

\begin{tabular}{|lccccc|}
\hline \multicolumn{7}{|l|}{$\begin{array}{l}\text { Table 1. } \\
\text { Means of horizontal perceptual change }\end{array}$} & \multicolumn{5}{l|}{} \\
\hline Condition & Horizontal & H. E. & Effect & Time to return to & Time to \\
& Difference & Difference & size & baseline (H match) & baseline (H.E.) \\
\hline 5 minutes & 0.07 & 0.06 & 0.49 & 0 & 0.33 \\
15 minutes & 0.10 & 0.14 & 1.02 & 10 & 5 \\
30 minutes & 0.19 & 0.27 & 1.35 & 10.67 & 9.67 \\
45 minutes & 0.17 & 0.26 & 1.06 & 13.33 & 14 \\
1 hour & 0.15 & 0.28 & 1.39 & 21.67 & 22 \\
2 hours & 0.18 & 0.33 & 1.58 & 23.33 & 25 \\
3 hours & 0.19 & 0.26 & 1.76 & 27 & 20.67
\end{tabular}




\begin{tabular}{|lrrrrr|}
4 hours & 0.21 & 0.33 & 1.88 & 28.33 & 21 \\
\hline
\end{tabular}

$\mathrm{H}$ match indicates horizontal match value and H.E. indicates horizontal effect score.
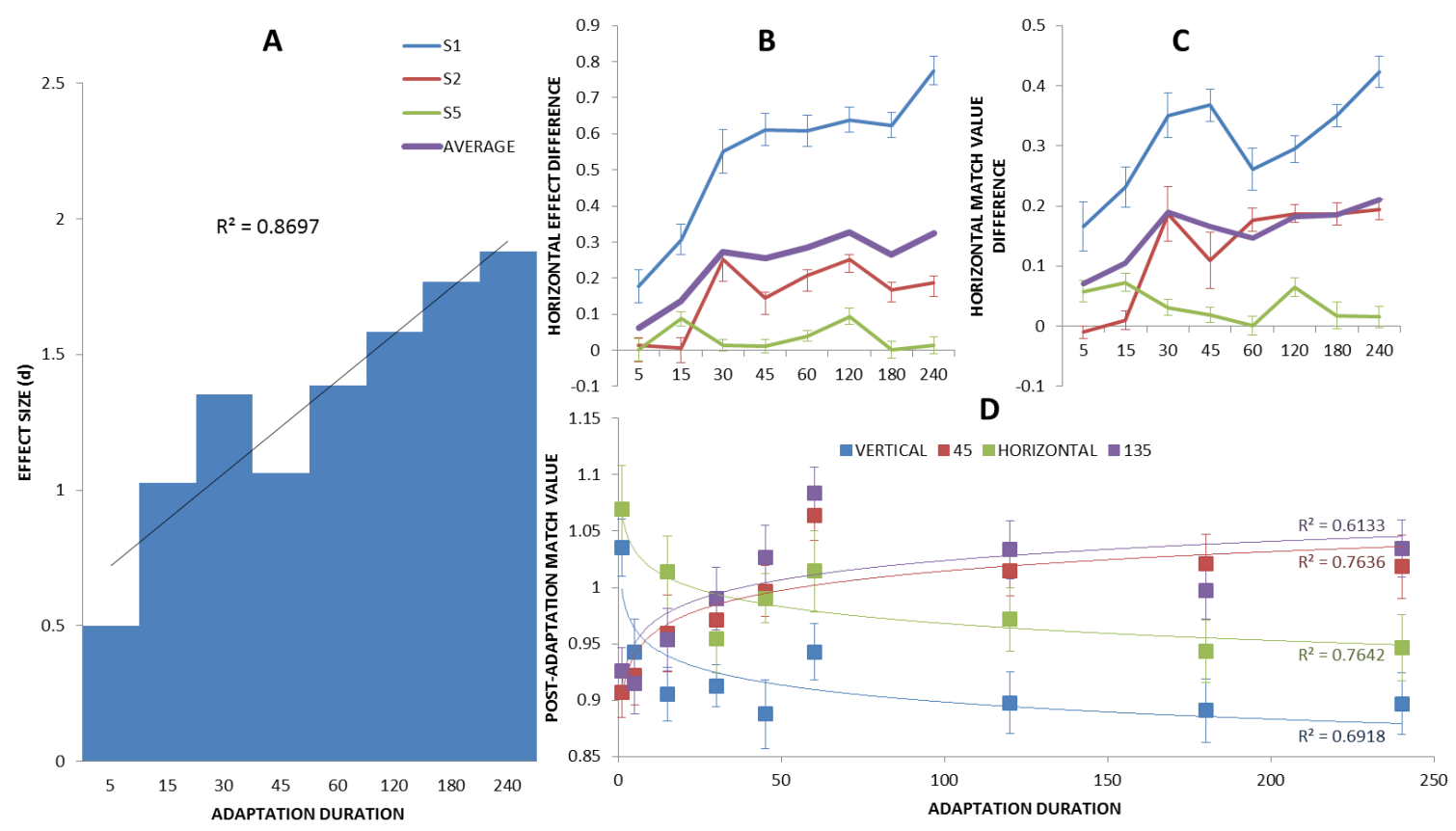

Figure 5. Summarized results of Experiment 1. A: effect size $(d)$ as a function of adaptation duration. B: Horizontal match value difference scores for each subject and the average (purple). C: Horizontal effect score for each subject and the average (purple). Error bars represent each subject's standard error. D plots the average post-adaptation match values across the four test orientations at each adaptation duration. Error bars here represent the average of each subject's standard error.

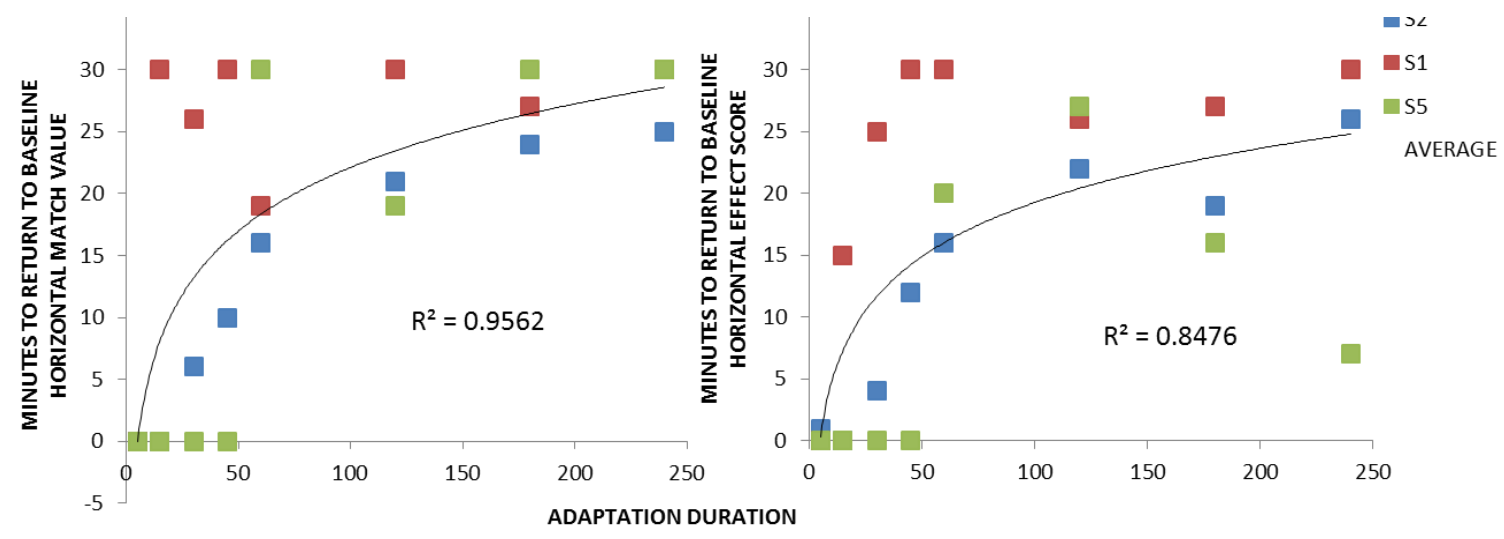

Figure 6. The average time it takes both post-adaptation horizontal effect score and horizontal match value to return to within one standard deviation of baseline across adaptation conditions with logarithmic fits for each average (black line). 
A

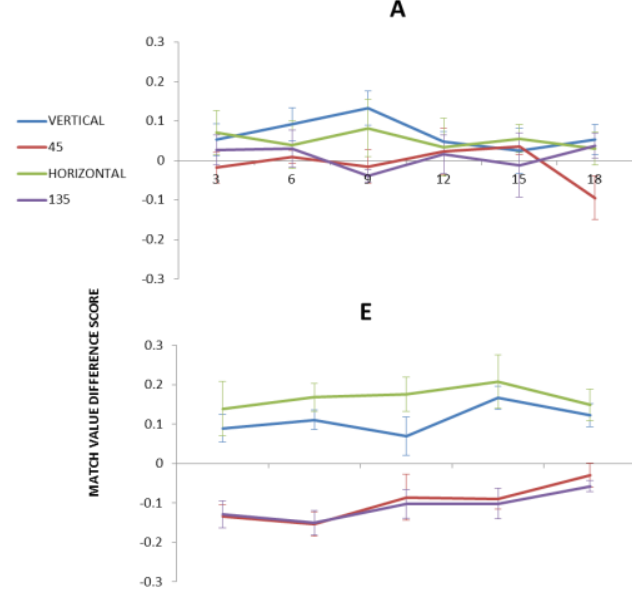

B

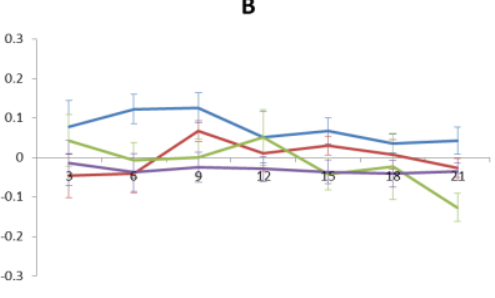

$\mathbf{F}$

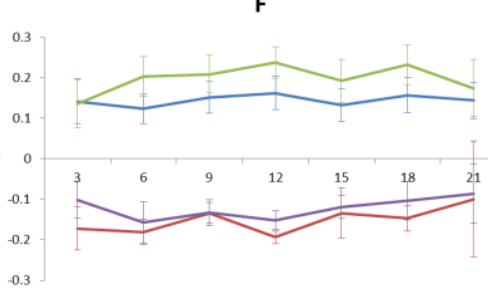

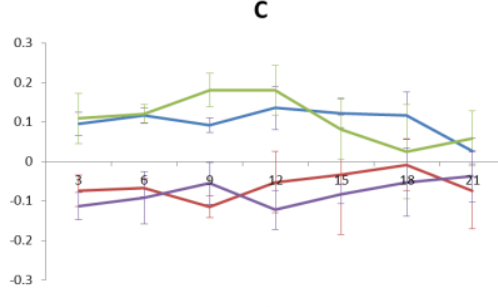

G

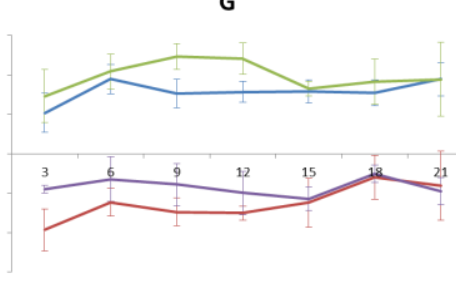

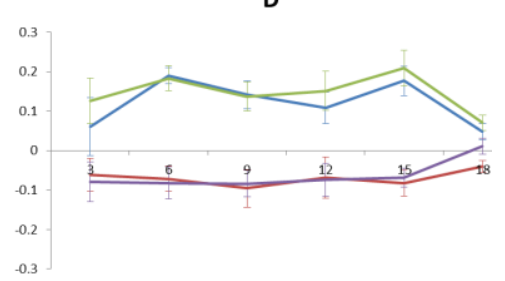

H

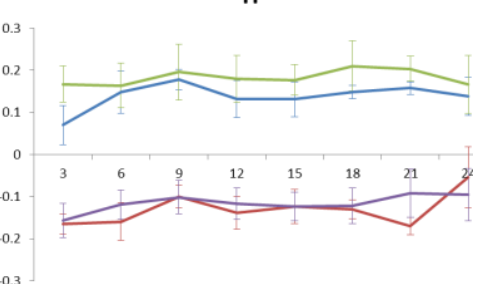

Figure 7. Average difference scores (pre-posttest) for each test orientation plotted across post-adaptation trial block (averaged in windows of 3 blocks). A-H correspond to the different durations of adaptation (5-240 minutes). Error bars represent the average of each subject's standard error. 
As can be seen in Figure 5, the shorter conditions (5, 15, 30, and 45 minutes) show substantial increases in perceptual change with increased adaptation duration. The average decrease in horizontal and vertical post-adaptation match values is not linear and so has been fit with a logarithmic function. That is, if one takes the logarithm of the adaptation time in minutes, there is a linear relationship between adaptation duration and the change in post-adaptation match values. The change starts with dramatic decreases from baseline (1) to $30 \mathrm{~min}$ of adaptation and then leveling off starting at 45 minutes of adaptation, $r^{2}=.76, p<.001$ for horizontal, .69, $p=.005$ for vertical. Moreover, the horizontal effect difference score and the horizontal match value difference score both increase after up to 45 minutes of adaptation (Figure 5). On the other hand, the average increase in the oblique post adaptation match values also appears to be logarithmic with dramatic increases from baseline to 60 minutes of adaptation and a plateau starting at 2 hours, $r^{2}=.76, p=.002$ for $45^{\circ}, r^{2}=.61, p=.013$ for $135^{\circ}$. The leveling out of the magnitude of perceptual change beyond 60 minutes carries over into the duration of effects as well; as can been seen in Table 1 and Figure 6, the relationship between adaptation duration and both the horizontal effect change and the horizontal match value change is also logarithmic, $r^{2}=.95, p>.001$ for horizontal effect, $r^{2}=.85, p=.001$ for match value. Lastly, effect size shows a linear relationship to adaptation duration: the average difference between horizontal match values before and after adaptation, when compared to the pooled error for these values increases monotonically with adaptation duration. Clearly, the duration of adaptation has an effect on the extent of perceptual change. Here the effect has been fit using a logarithmic function, although further testing would be necessary to completely determine the exact relationship. 


\section{EXPERIMENT 2: Magnitude of change on adaptation effects}

\section{Methods}

The next experiment examined how strong the physical anisotropy in the adapting stimulus needed to be in order to change orientation perception as measured by the matching task. Since the two-hour adaptation condition showed optimal effects, the duration of adaptation was fixed (for Experiment 2 and all subsequent experiments) and the magnitude of change that the filter imposed on the subjects' environment differed. That is, the multiplicative scalar used to decrease the horizontal content of the image was varied. Whereas in Experiment 1 the scalar was fixed to increase or decrease oriented content by $85 \%$, in Experiment 2 scalars that decreased content by $65 \%$ and $35 \%$ were tested for comparison (see Figure 8). Otherwise, the filter and adaptation parameters were exactly the same as those used in Experiment 1. Here, like in Experiment 1, the magnitude of filter change was quantitatively compared to the magnitude of perceptual change using difference score of horizontal match value, horizontal effect score, and effect size. The same 4 subjects (S1-S4, 2 female, 3 naive) adapted to environments altered with each of the scalars for 2 hours (S1 and S2 new scalar values were compared to those obtained in Experiment 1). Subjects were assigned to experience the different scalars in random order. 


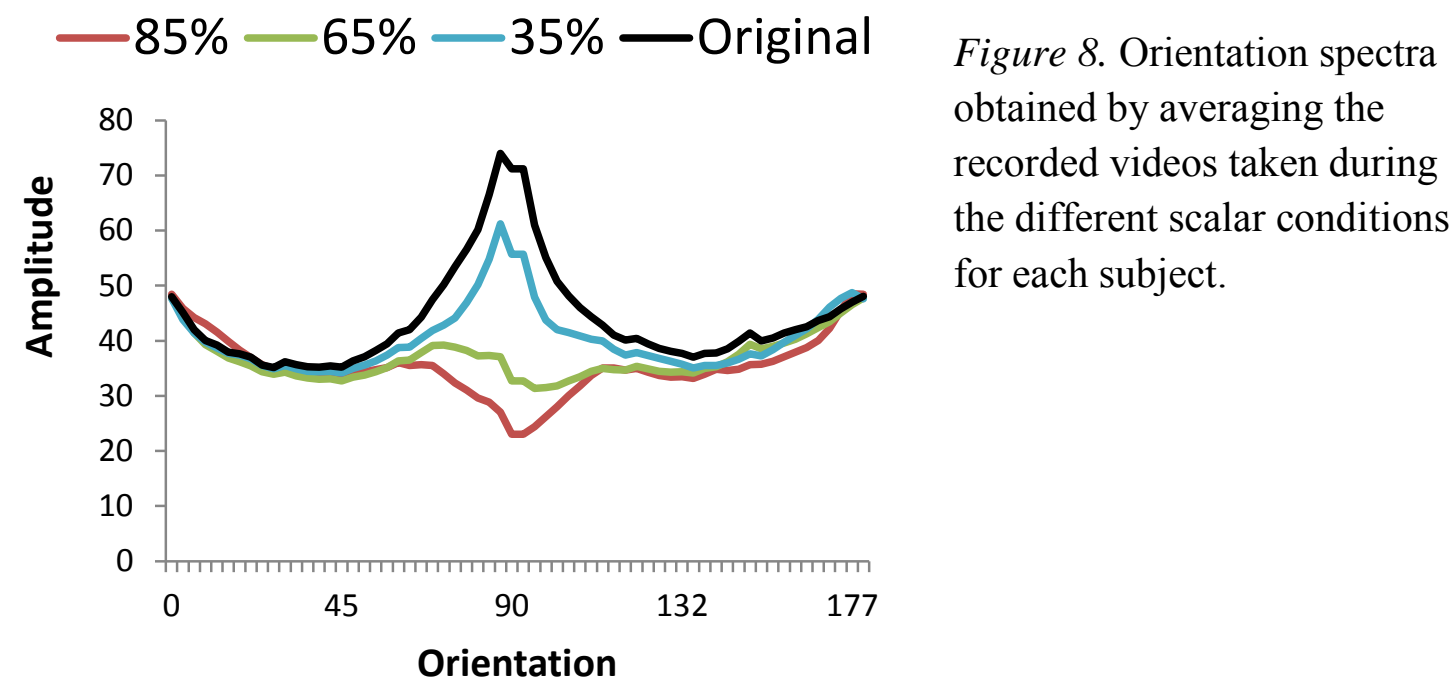

Results

Again, the one way within subjects ANOVA comparing horizontal match value differences was not significant, presumably due to being underpowered, $F(2,6)=3.25, p$ $=.11$. However, all three measures of perceptual change indicate that the more horizontal content in the environment is decremented, the more sensitive subjects become to horizontal orientations. The difference between pre and post-test (first ten minutes) horizontal match values as well as the effect size steadily decreases as the scalar increases. The difference in the horizontal effect score does not monotonically increase with decreasing scalar, but it does increase dramatically from $35 \%$ to $65 \%$.

\begin{tabular}{|c|c|c|c|}
\hline \multicolumn{4}{|c|}{$\begin{array}{l}\text { Table } 2 . \\
\text { Means of horizontal perceptual change across magnitude conditions. }\end{array}$} \\
\hline $\begin{array}{l}\text { Physical } \\
\text { change }\end{array}$ & $\begin{array}{l}\text { Horizontal } \\
\text { Match Difference }\end{array}$ & $\begin{array}{l}\text { Horizontal Effect } \\
\text { Difference }\end{array}$ & $\begin{array}{l}\text { Effect } \\
\text { Size }\end{array}$ \\
\hline $85 \%$ & 0.172698 & 0.160443 & 2.892735 \\
\hline $65 \%$ & 0.102778 & 0.166616 & 2.401152 \\
\hline $35 \%$ & -0.12778 & 0.027759 & 0.153585 \\
\hline
\end{tabular}



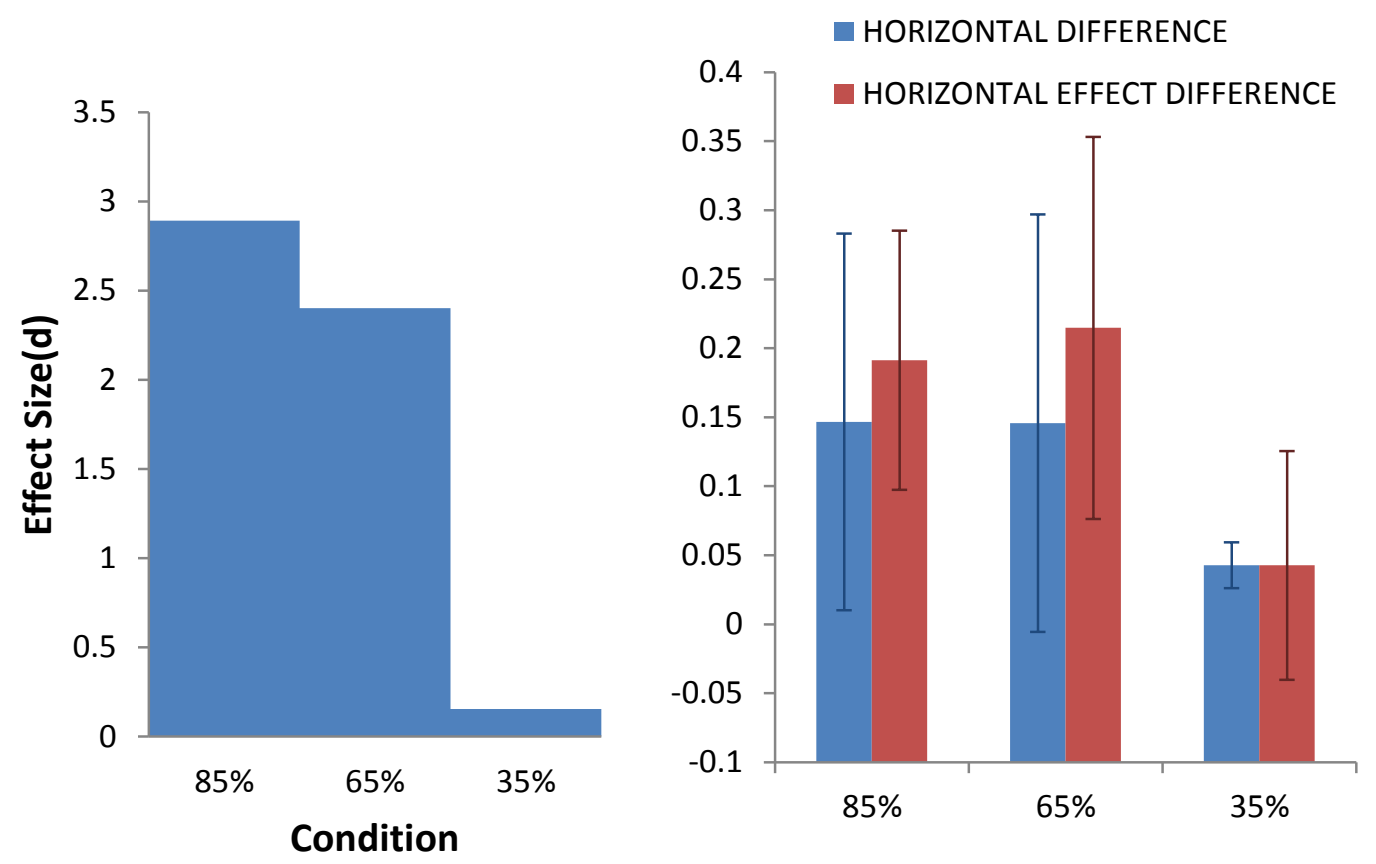

Figure 9. Measures of horizontal perceptual change across magnitude of physical change. Left shows effect size and right shows horizontal match value and horizontal effect size difference scores (pre-post). Error bars represent standard error.

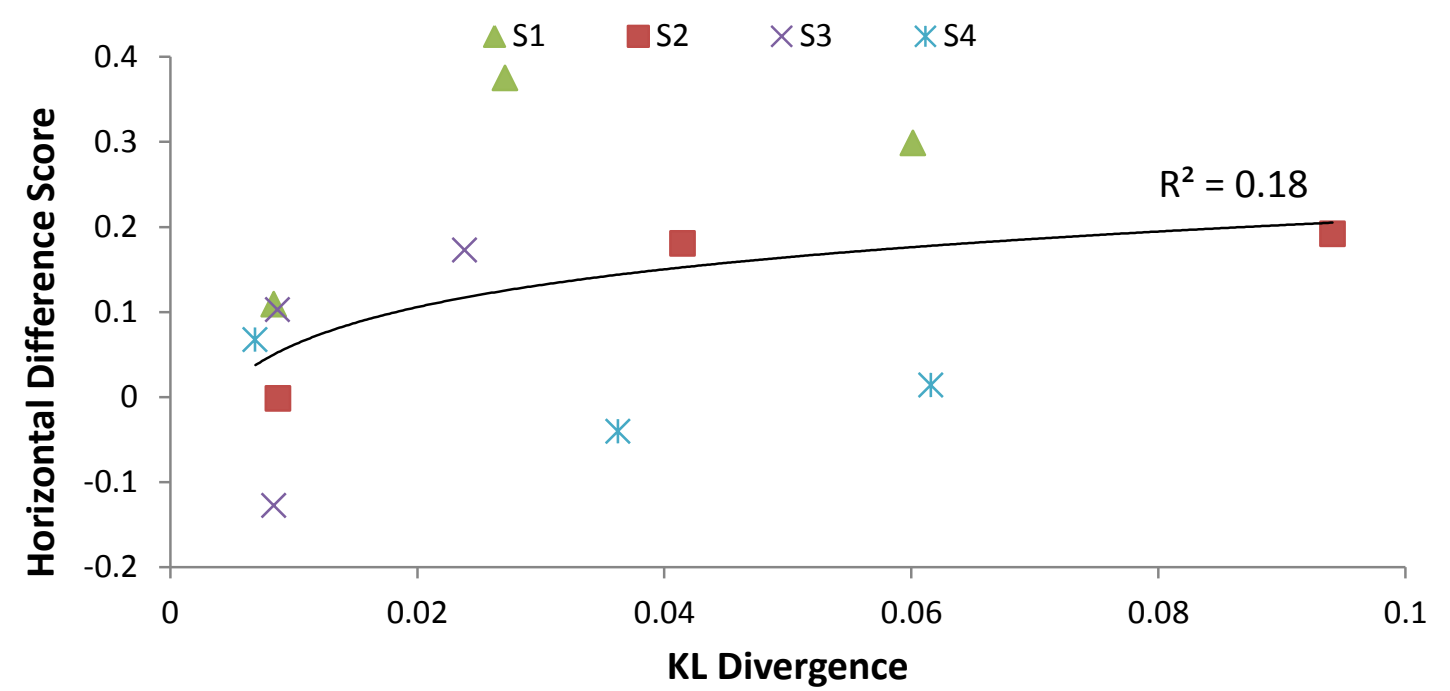

Figure 10. Each subject's horizontal difference score plotted as a function of KL divergence. The mean shows a weak logarithmic relationship. 


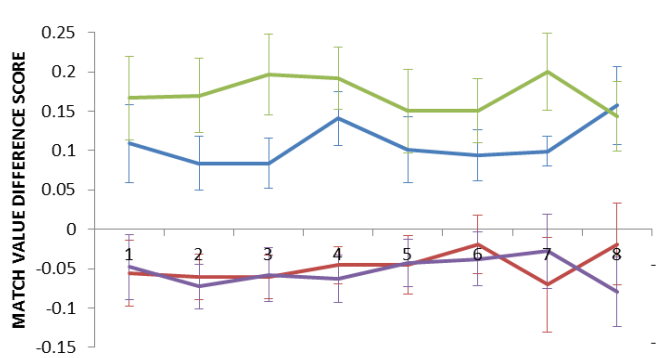

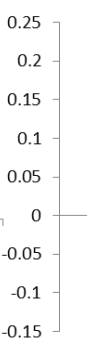

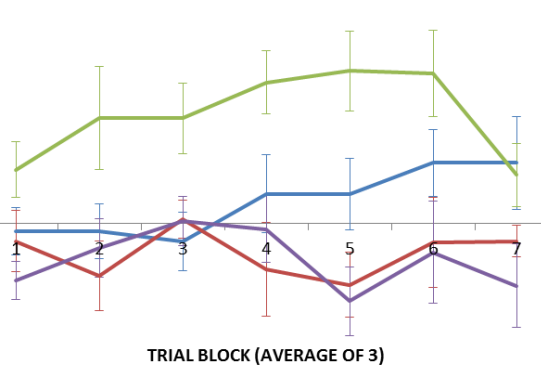

C

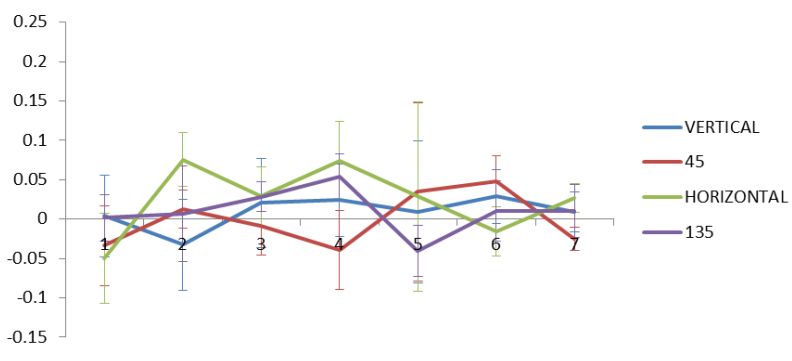

Figure 11. Median difference scores (pre-posttest) for each test orientation plotted across post-adaptation trial block (averaged in windows of 3 blocks). A-C correspond to 35\%,65\%, and 85\% change respectively. Error bars represent the average of each subject's standard error. 
It appears that the specific orientation being physically changed in the environment must be decremented to at least $65 \%$ of its original value in order for a perceptual change to occur. Whereas the $35 \%$ change induced almost no noticeable change in perception, both the $65 \%$ and $85 \%$ change induced a substantial change in perception (see Figure 9). This makes sense when you compare the physical change induced by the difference scalars as plotted in Figure 8 . The 35\% scalar does not induce much perceptual change because the shape of the natural anisotropy is retained, just to a lesser extent. The dramatic jump in perceptual change as a result of the $65 \%$ decrement is likely due to the complete lack of a horizontal peak in this environment (Figure 8). The even stronger change at $85 \%$ can be attributed to the decrement at horizontal in this environment. In other words, whereas the $35 \%$ change is still a fairly anisotropic environment, both the $65 \%$ and $85 \%$ scalars completely removed the horizontal peak. The increase in perceptual change from $85 \%$ to $65 \%$ physical change is not nearly as dramatic as the increase from $35 \%$ to $65 \%$. These findings indicate that the strength of perceptual change is indeed related to the strength of the physical change. To test this finding more explicitly, the horizontal difference scores for each subject were plotted against the specific KL divergence scores computed from the video recordings made for each magnitude change (Figure 10). In this way, the relationship between horizontal perceptual change and the difference between the experienced environmental distribution and the natural one could be determined. The best fit relationship between these data was indeed logarithmic, although the relationship was not significant, $r^{2}=.18, p=.16$. Smaller increments of physical change would be necessary to determine the exact 
relationship between physical change and perceptual change, although it does not appear to be linear.

What is clear in these data as well as in the data from Experiment 1 is that there appears to be a relationship between the perceptual change of horizontal and vertical orientations. Close inspection of Figures 7 and 11 shows that, in conditions where the horizontal match value is changing greatly between pre and posttest, the vertical difference score is also high. Moreover, it appears that, as both adaptation duration and the magnitude of physical change increase, the pairing of horizontal and vertical also increases. That is, as one moves from A-H in Figure 7 or A-C in Figure 11, the pairing of the cardinal orientations gets stronger. This is particularly evident in Figure $8 \mathrm{C}$ to D and in Figure 11 A to C. In fact, whereas in the $35 \%$ condition (Figure 11C) there appears to be no relationship between vertical and horizontal difference scores, the relationship increases in $65 \%$ (11B) and shows a strong association in $85 \%(11 \mathrm{~A})$. A similar, if less clear trend can be seen as one move from 30 minutes of adaptation (Figure 7C) to 2 hours (Figure 7F). The pairing of perceptual change for the cardinal orientation was unexpected and cannot be directly related to the change in the environment. This relationship is further examined in follow-up tests (below). 


\section{EXPERIMENT 3: Immersive versus Passive Viewing}

\section{Methods}

The last horizontal deprivation paradigm involved viewers watching filtered video feeds of the environment while sitting still in the lab. These videos were recorded and filtered in real-time, but the camera was not mounted to the front of the observer's head, thus eliminating the feedback that an observer received while wearing the HMD and camera together. In one condition, viewers watched the filtered videos through the HMD to control for differences in viewing stimuli with and without the HMD. Therefore, the only difference between this and the other conditions was the concurrent interaction with the environment. Any differences in adaptation experience could thus be attributed to the experiential feedback and real-time viewing. In second type of passive condition, subjects viewed the filtered videos on a computer monitor to control for differences in wearing the HMD compared to simply viewing the filtered environment. During both types of passive experience, subjects viewed a filtered movie for the entire adaptation period ( 2 hours). While passively viewing over a monitor, subjects were isolated in a dark room in which the only source of visual information was coming from the computer screen. Both types of passive viewing as well as the immersive adaptation condition for this paradigm consisted of the optimal magnitude and duration parameters determined from Experiments 1 and 2 (horizontal at $85 \%$ decrement for two hours). The same 4 
subjects participated in this experiment as in Experiment 2 and the two passive conditions were compared to their immersive condition from Experiment 1 and 2.

\section{Results}

As in the above experiments, the one-way ANOVA comparing horizontal difference scores across the three types of adaptation experience was underpowered, $F(2,6)=.87, p=.47$. Still, all three measures of perceptual change decreased when the subject is still immersed in the environment, but not getting feedback in real time (Passive 1; Table 3). Moreover, passive experience on a monitor induces little if any perceptual change as measured by effect size (Figure 12). As in Experiments 1 and 2, there is an association between the perceptual change at horizontal and vertical which increases as the experience becomes more immersive (Figure 13A-C). Again, this relationship is the subject of the follow-up tests discussed below.

\begin{tabular}{|lccc|}
\hline Table 3 & & & \\
\hline Condition & $\begin{array}{c}\text { Horizontal Match } \\
\text { Difference }\end{array}$ & $\begin{array}{l}\text { Horizontal Effect } \\
\text { Difference Score }\end{array}$ & Effect Size \\
\hline Immersive (from Experiment 1) & 0.17 & 0.24 & 2.89 \\
Passive 1 (through HMD) & 0.14 & 0.22 & 1.26 \\
Passive 2 (on a monitor) & 0.08 & 0.19 & 1.11 \\
\hline
\end{tabular}
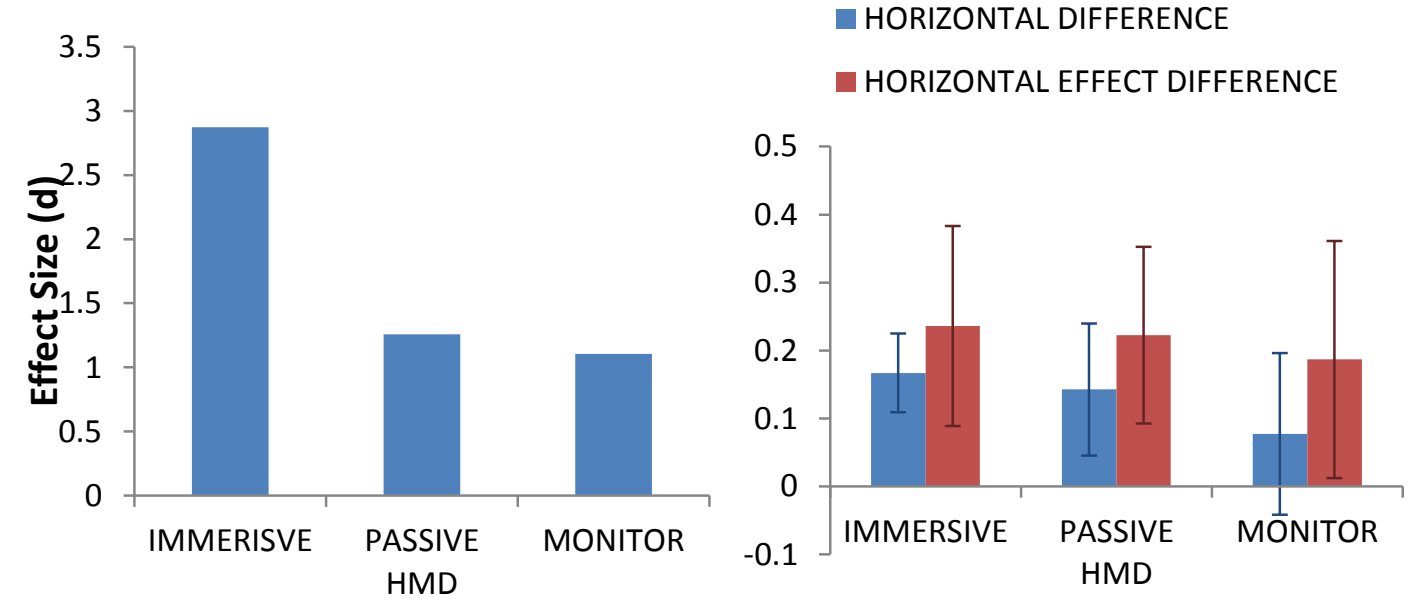

Figure 12. Measures of horizontal change across adaptation type. Error bars represent standard error. 
A

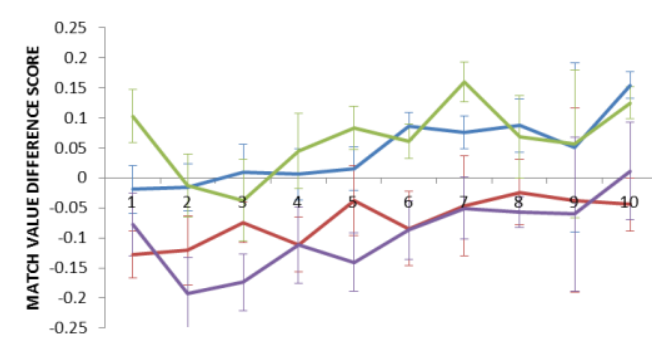

B

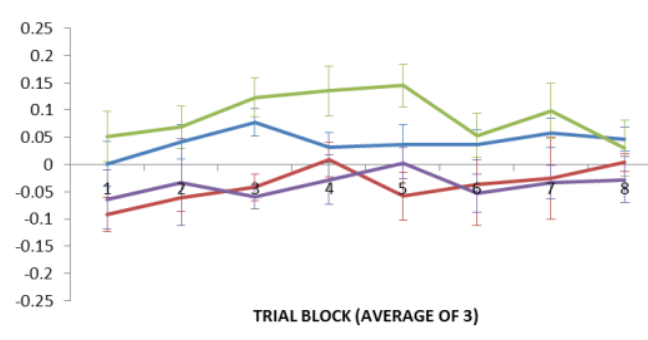

C

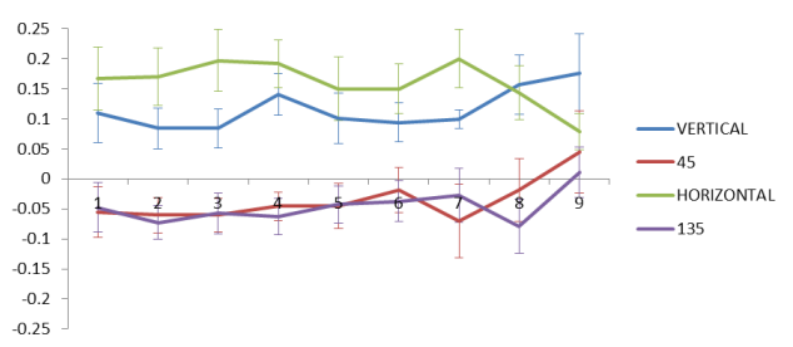

Figure 13. Median difference scores (pre-posttest) for each test orientation plotted across post-adaptation trial block (averaged in windows of 3 blocks). A-C correspond to the monitor, passive HMD, and immersive viewing conditions respectively. Error bars represent the average of each subject's standard error. 
EXPERIMENT 4: Examining the Effects of an Isotropic Environment

\section{Methods}

As stated above, the primary goal of this project was to determine if recent experience fully or partially accounts for the horizontal effect typically observed in broadband orientation perception. Can the horizontal effect that is usually observed in subjects be attributed to horizontally biased environmental experience? As a step towards answering this question, the goal of this experiment was to determine whether extended experience in an isotropic environment could completely eliminate the horizontal effect bias. The image that the subjects viewed through the HMD was altered to 'undo' the typical orientation bias present in natural images. That is, unlike previous research (and unlike Experiments 1-3) the amplitude spectra of the images was not filtered, but rather replaced with an isotropic spectrum with the same $1 / f$ property of typical natural scenes. The isotropic amplitude spectrum was created in Matlab by exponentially ramping down the amplitude values from the center outwards radially (see Figure 14C). Thus, as in other adaptation paradigms, each frame of the video output was fast Fourier transformed (FFT) and the resulting amplitude spectrum was replaced with a naturalistic $(1 / f)$, but isotropic spectrum (see Figure 14). The isotropic spectrum was then convolved with the original phase spectrum of each frame and inverse transformed to be presented on the 
HMD. Substituting the isotropic spectrum for the original amplitude maintained the basic structure of the image (as structural relations are determined largely by the phase spectra: Morgan, Ross, \& Hayes, 1991; Oppenheim \& Lim, 1981; Piotrowski \& Campbell, 1982; Shapley et al., 1990) but ensured an equal amount of structure (amplitude) at all orientations at each spatial frequency. Nine subjects (including S1-S5, 4 female, 8 naïve, $M=23.6$ years) participated in the main experiment. Subjects adapted to the isotropic environment for 2 hours and then completed the post-test $(30 \mathrm{~min})$ to determine if the horizontal effect had been decreased or abolished in comparison to their pre-test.

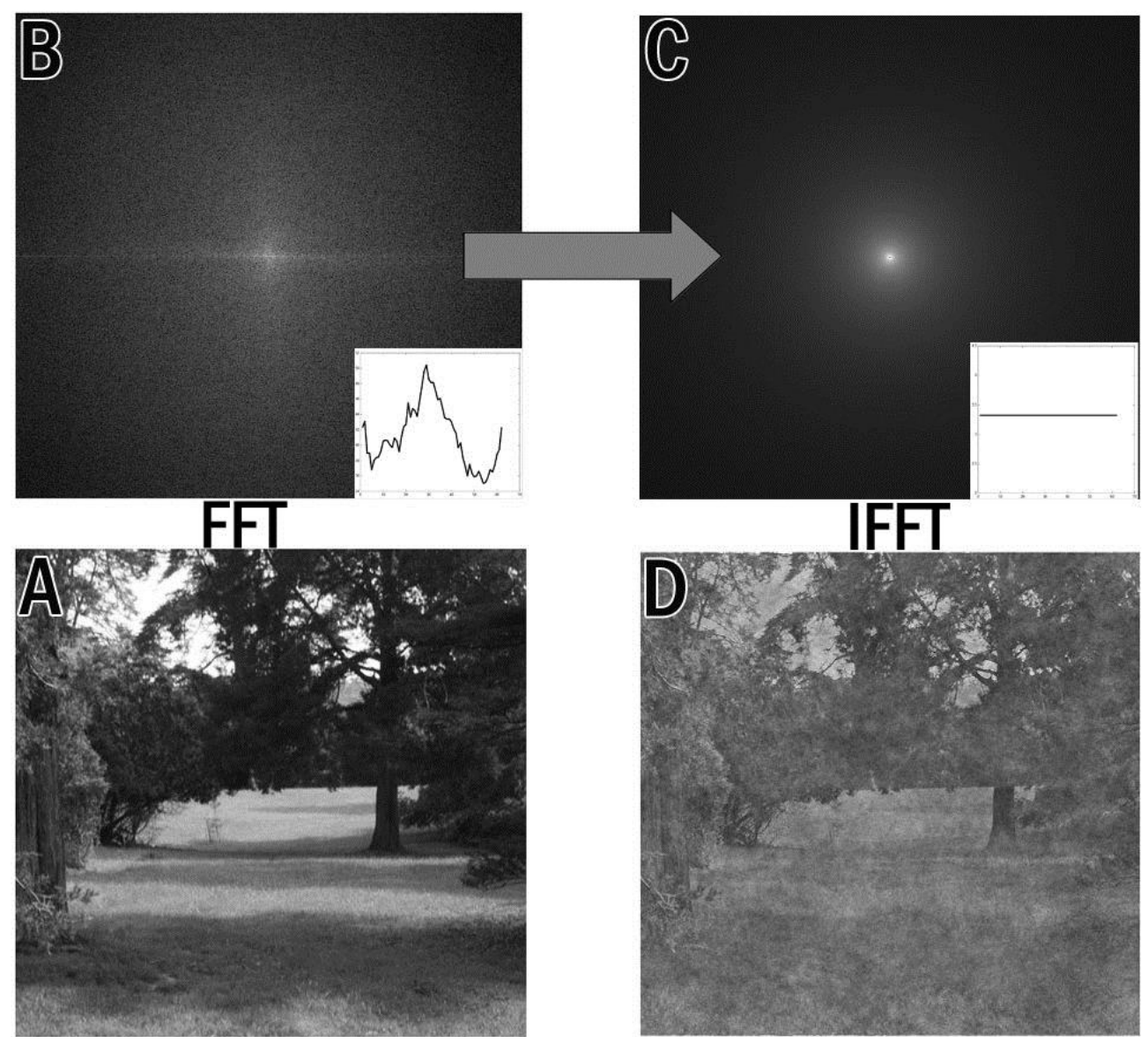

Figure 14. Example of the filtering processing for Experiment 4. The image captured by the camera (A) is transformed (FFT) and its amplitude spectrum (B) replaced with an isotropic spectra (c). The process is then inverted and the resulting isotropic image (D) is sent to the HMD for display. 
A subset of subjects ( $\mathrm{n}=3: \mathrm{S} 2, \mathrm{~S} 5$, and $\mathrm{S} 6,2$ female, 2 naive) participated in two alternate adaptation conditions that also changed the global distribution: 'oblique dominant' and 'enhanced natural.' In the oblique-dominant condition, the typical natural anisotropy $(\mathrm{H}>\mathrm{V}>>\mathrm{O})$ was altered so that the distribution peaked at the obliques and exhibited minima at the cardinals. The filter used during this adaptation consisted of a triangular in orientation filter (like Experiments 1-3) that attenuated cardinal and enhanced oblique amplitudes (equally at all spatial frequencies) within $45^{\circ}$-wide bands of orientations (see Figure 15). For the oblique-dominant condition the $45^{\circ}$-wide triangles had a peak (maximum) amplitude of a factor of 1.95 at $45^{\circ}$ and $135^{\circ}$ and a peak (minimum) amplitude of a factor of 0.15 at $0^{\circ}$ and $90^{\circ}$. For the enhanced natural condition the filter had peaks of 1.95 at $0^{\circ}$ and $90^{\circ}$ and minima of 0.35 at $45^{\circ}$ and $135^{\circ}$. An example of each filtering process can be seen in Figure 15. Just as before, after adaptation, subjects completed as many matching trials as possible during a 30 minute post-test.
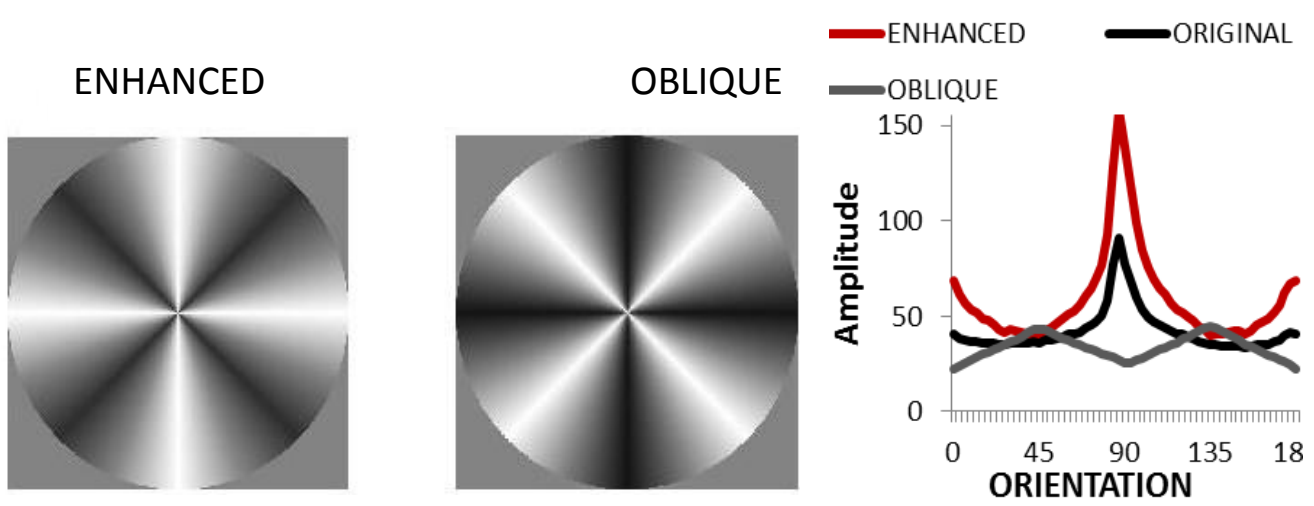

Figure 15. Filters used for the inverted and enhanced conditions shown in both two dimensions (left) and one dimension (right). 


\section{Results}

The addition of new, less experienced subjects increased the overall variability in the data. To compensate for this change, only the first five minutes of post-testing (instead of ten) are included in all analyses for the isotropic condition in Experiment 4. Additionally, less experienced observers were asked to repeat the experiment in order to obtain less variable data. All subjects who were able to repeat the experiment were run as many times as possible and the average of the last two runs was taken (S1, S6, S7, and S9). As in Experiments 1-3, observer's average match values before adaptation were compared to the match values obtained after adaptation. As seen in Figure 16, the horizontal effect present in the baseline condition became quite flat after experience in the isotropic environment. A 2 (baseline vs beginning of post-test) x 4 (test orientation) two-way repeated measures ANOVA was run on subjects' match values to determine if adaptation in an isotropic environment affected match values differently by orientation. The within-subjects analysis showed a significant interaction between orientation and testing phase, $F(3,48)=11.05, \mathrm{p}<.001$. The salience at horizontal increased and decreased at vertical (although only horizontal change was significant, $p=.05$ while vertical $p=.11$ ). Moreover, sensitivity to both of the obliques decreased (but only the $45^{\circ}$ change was significant, $p=.002, p=.15$ see Figure 16$)$. 


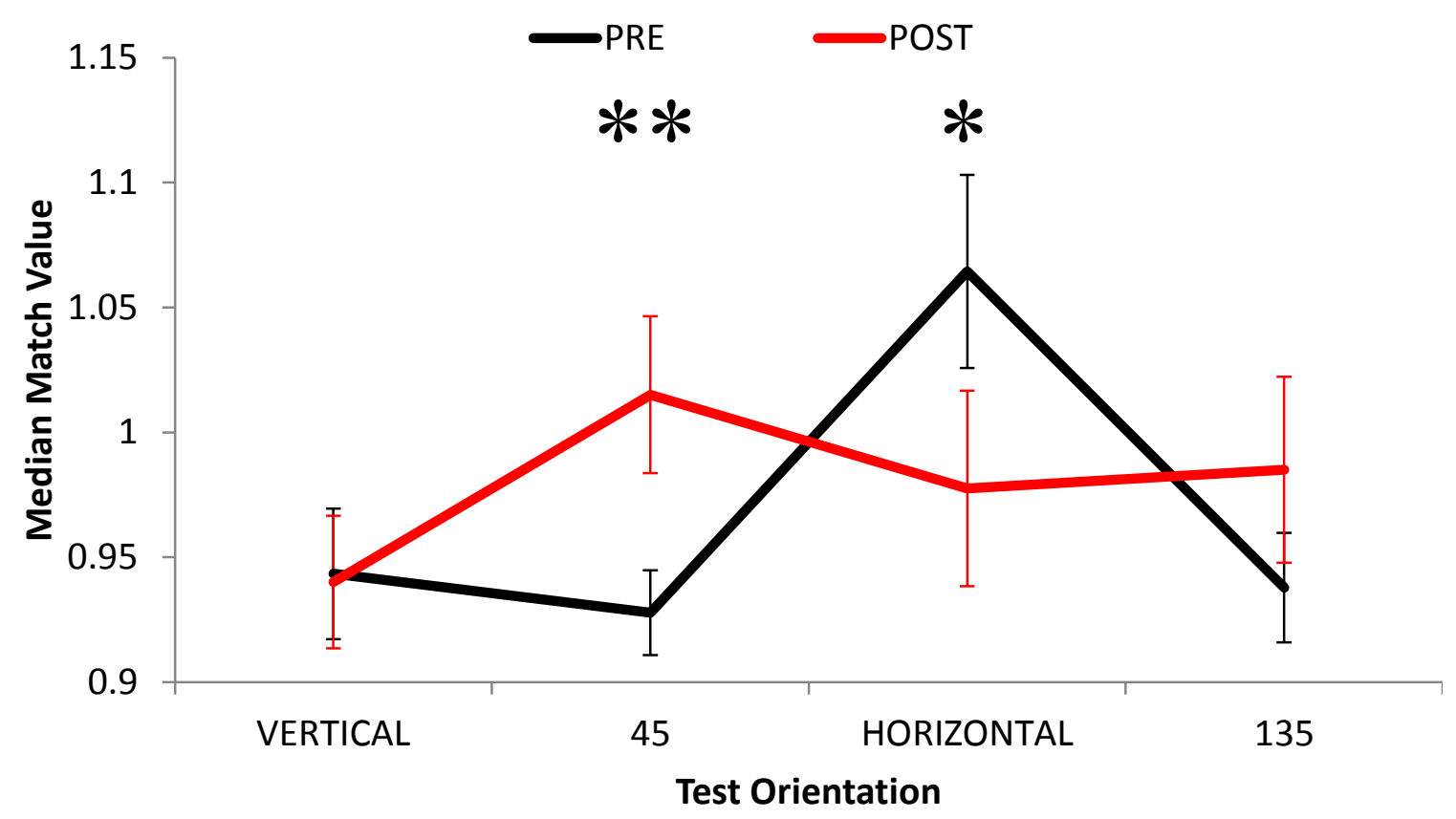

Figure 16. Average pre (black) and post (red) adaptation median match values across test orientation. Error bars show standard error and * indicate significant differences between pre and post-test values.

During the post-test period, each subject's match values changed, although to differing extents. To quantify this change, the same horizontal effect score measure was calculated pre and post (five min) adaptation. The horizontal effect score for each subject as well as the average is plotted in Figure 17. As can be seen, all subjects showed a decrease in this measure of perceptual anisotropy after adaptation. That is, subjects showed a more isotropic pattern of orientation perception after adapting to an isotropic environment for 2 hours $(p=0.006)$. 


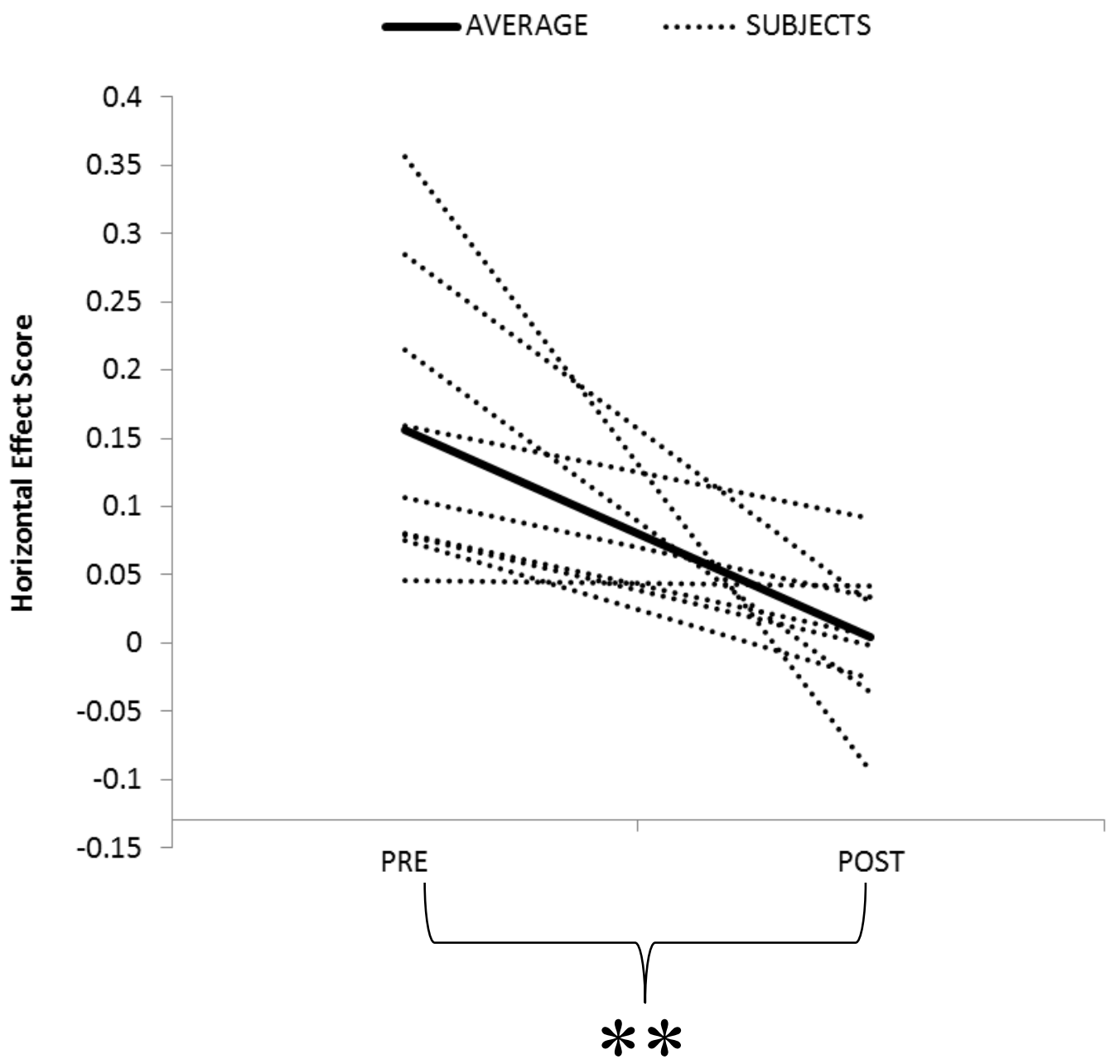

Figure 17. Pre and post-test horizontal effect scores on average (solid) and for each subject (dashed). * indicates significance.

Results from the enhanced and inverted conditions are plotted in Figure 18. For comparison, the data for these three subjects after adaptation in the isotropic condition has been plotted as well. The horizontal effect difference score was again calculated as a measure of quantitative change. Observers' orientation perception changed after experience in both of these altered environments. In the oblique condition, subjects 
became more sensitive to the cardinal orientations and less sensitive to the obliques. In the enhanced natural condition, subjects became even less sensitive at the cardinal orientations and slightly more sensitive at the obliques effectively increasing the horizontal effect. Moreover, the horizontal effect changes across the three types of altered environments: it is highest in the super-natural condition and lowest in the isotropic condition.

As in Experiment 2, subject's difference scores (pre-post) were compared with the KL divergence scores obtained from the unfiltered and filtered videos to determine the relationship between the physical and perceptual differences. Since the isotropic filter changes the amplitude content of every orientation in the image, the difference scores for each tested orientation were used for comparison. As can be seen in Figure 19, the cardinal orientations showed a weak positive relationship to KL divergence while the obliques showed effectively no relationship. None of the correlations were significant. To compare the decay of perceptual effects across adaptation to global distribution changes, average match values (across all subjects) were computed in five minute intervals across the post-testing period (Figure 20). Interestingly, the first five minute interval seems to show the predictable pattern for each condition: isotropic (B) shows all orientations moving towards equality, Enhanced Natural (A) shows the opposite, and Oblique Dominant (C) shows an increase in sensitivity for the cardinals and a decrease for the obliques. However, these predictable patterns only last the first five minutes and effects decay to baseline fastest in the Enhanced Natural condition and slowest in the Oblique Dominant condition. 

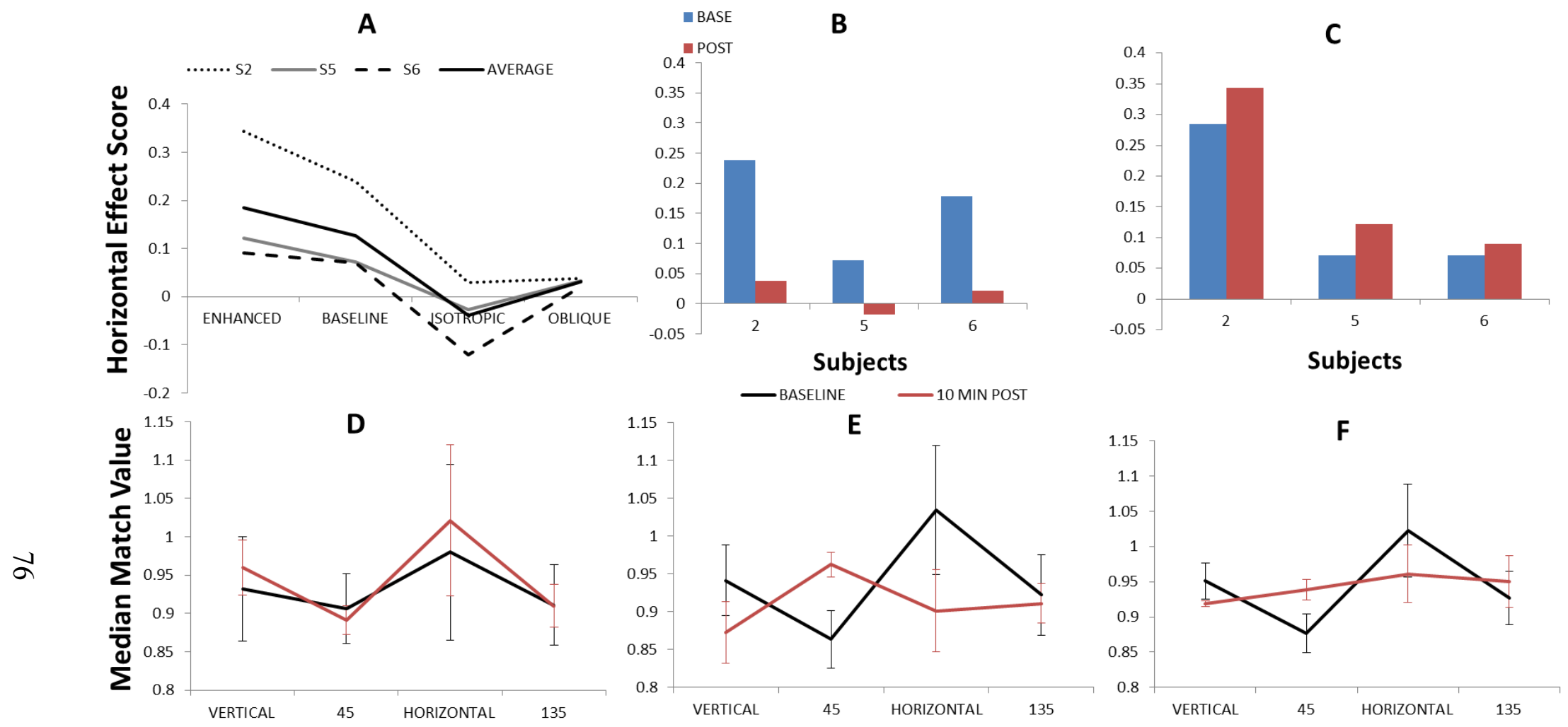

Figure 18. Results of the enhanced natural (C and D) and oblique dominant (B and E) adaptation conditions. A shows the horizontal effect score calculated for the first 10 minutes of post-testing for each subject and the average across all three adaptation conditions in comparison to baseline. The difference between the baseline and the post-test horizontal effect score for each subject is plotted in B and C. D-F show the average match values across test orientation for each adaptation condition. 
A

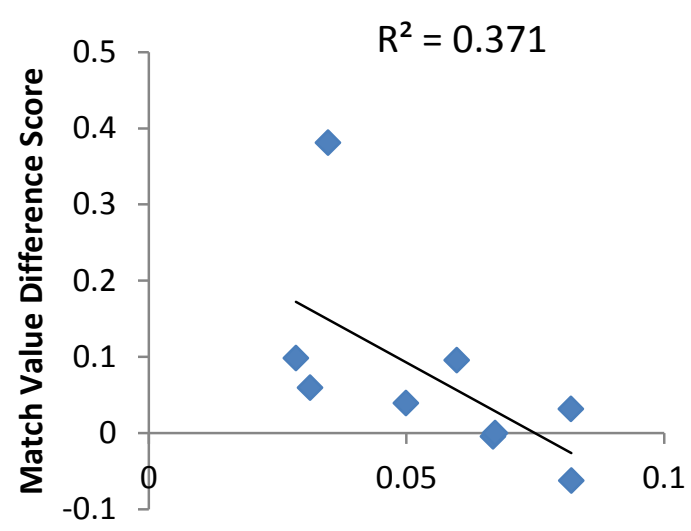

C

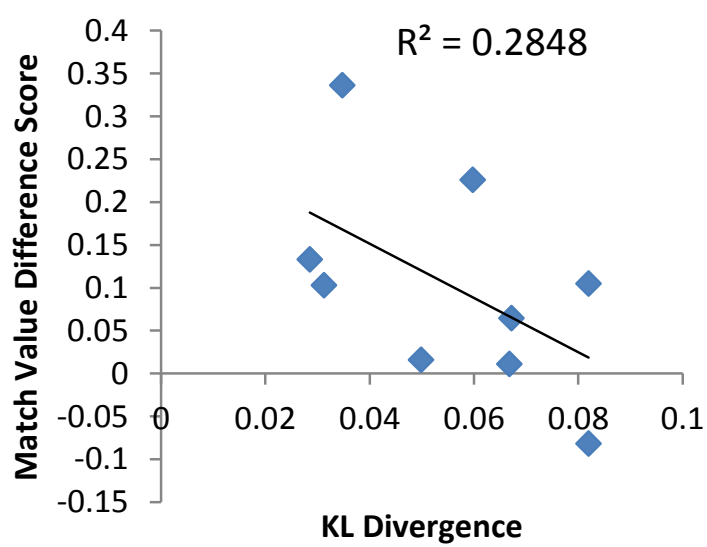

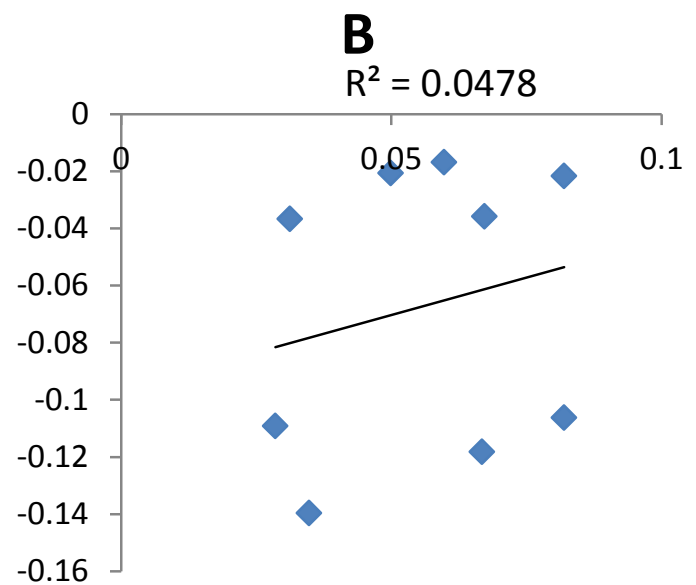

D

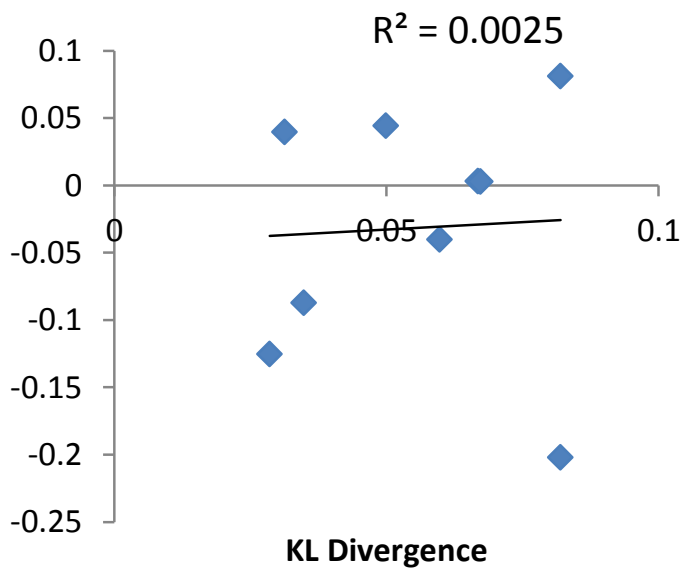

Figure 19. The relationship between KL divergence and the difference scores (pre-post) for each test orientation $(\mathrm{A}=$ vertical, $\mathrm{B}=45, \mathrm{C}=$ horizontal, $\mathrm{D}=135)$ after isotropic adaptation is plotted for each subject. Cardinal relationships appear to be linear. 


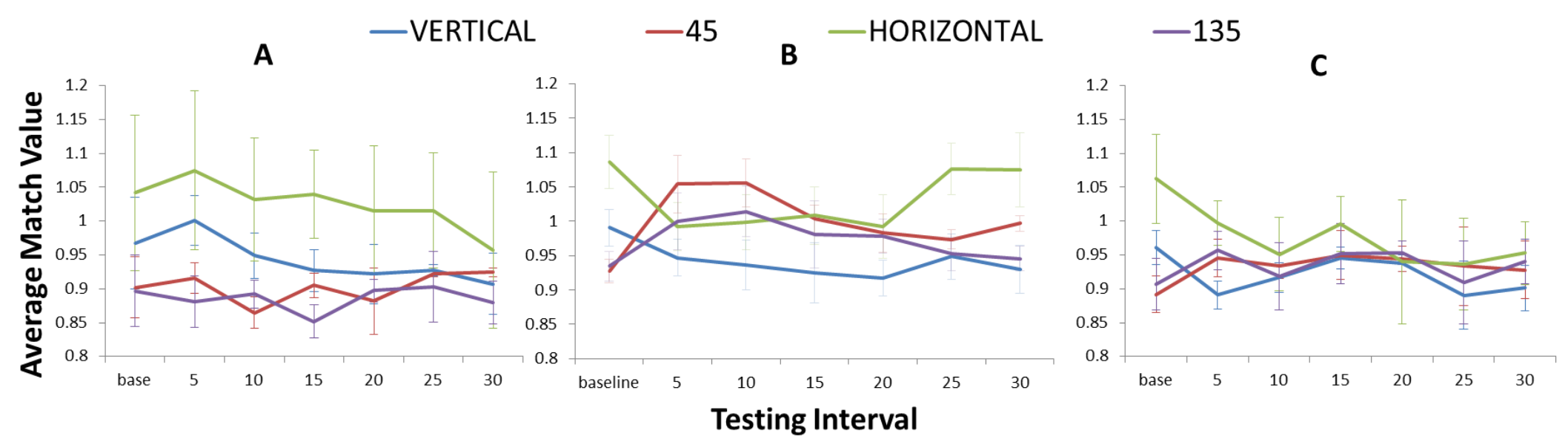

Figure 20. Average change for all subjects tested across post-test period for all three global adaptation conditions: Enhanced Natural ( $\mathrm{A}, \mathrm{N}=3)$, Isotropic $(\mathrm{B}, \mathrm{N}=9)$, and Oblique Dominant $(\mathrm{C}, \mathrm{N}=3)$. post-testing period is measured in5 minutes intervals with baseline scores plotted for reference on the far left of the abscissa. Error bars represent standard error. 


\section{CONTROL TESTS}

\section{Methods}

The filtering process does significantly alter the way an image looks to such an extent that observers report noticeable changes and it takes some time before they are able to adjust to the filtered world (Bao \& Engel, 2012; Zhang et al., 2009). For example, Figure 15 shows an image before and after filtering the spectrum to be isotropic. The image on the far right looks 'weird' and one could imagine that it would be difficult to interact in such an environment. Moreover, one could argue that testing a subject after adapting to such an environment may lead to overall changes in orientation processing simply due to the difference in how the image looks (rather than the physical oriented structure present). Since the post-test period was fixed in terms of time, it also cannot be determined whether it is time outside of the altered HMD environment or visual experience in the unfiltered, post-adaptation environment that decays the effects of adaptation. Lastly, Experiments 1- 3 all focus on changing only orientations near horizontal while Experiment 4 focuses on the global distribution of orientations. However unlikely, the perceptual changes exhibited in Experiments 1-3 in response to changing particular orientations could be specific to changing horizontal content. In order to ensure that any differences found were indeed due to altered experience with 
specific orientations, what causes them return to baseline after post-test, and that the changes are generalizable across orientations, control experiments were executed in concert with those listed above. For the first control, 3 subjects (S1, S2, and S4, 2 female, 2 naive) wore the HMD and viewed the unfiltered world through the camera so that the delay and HMD viewing were the same, but no content in the environment had been altered. In another control, S2 wore the HMD for a medium duration (2 hours) from Experiment 1, but viewed images which had been filtered in real-time to alter their spatial frequency, not orientation content. In this way, it was ensured that any adaptationinduced changes in orientation processing were due to altered orientation experience rather than simply altered perception in general. To account for the decay of adaptation, 3 subjects (S1, S2, and S3, 2 female, 2 naive) adapted to a vertical decrement condition (the same as Experiment 1, but with the filter centered at vertical rather than horizontal) then sat in darkness for 30 minutes between adaptation and post-test. This delay in darkness served to control for the time delay between adaptation and the return to baseline seen at the end of the post-testing period, but observers had no visual input during the delay. To compare altering horizontal content to altering other orientations, the same 3 subjects also adapted to a vertical decrement without a delay and, with the addition of a fourth subject, an enhancement of oblique orientations for two hours. This condition was run with the same type of multiplicative, triangular filter, this time centered at $45^{\circ}$ and $135^{\circ}$ to increase these orientations by $85 \%$ ).

All of the KL divergence measures described in Experiments 2-4 above assume the pre-filtered video recording of the environment observers were exposed to during 
adaptation is a good measurement of the un-adapted environmental distribution, but it could be argued that observers may be exposed to different environmental orientation distributions before the pre-test (i.e., before they come in to run the experiment) that are not reflected in the orientation spectra of the pre-filtered recorded videos. To account for these differences the post-test from the unfiltered control was compared to the baseline run within subjects. If the environment experienced in the lab was significantly different than that experienced before the pre-test, the baseline should differ from this control posttest.

\section{Results}

Wearing the HMD for two hours with no filter, simply viewing the world in black and white caused no change in orientation perception: the horizontal effect persisted (Figure 21). Correlations between match values at the four test orientations were run to assess the match between orientation perception with and without a delay as well as before and after wearing the HMD with no filter. Wearing the HMD for 2 hours with no filter did not alter subjects' orientation perception in the post-test, $R^{2}=.96, p=.0003$. Although only one subject ran the spatial frequency filter condition, as can be seen from Figure 22, this did not affect this subject's orientation perception at all; S2 showed no difference between pre and post-test match values after adapting to an environment with altered spatial frequency content.

Adding a 30-minute delay between the end of adaptation and the beginning of post-testing also did not significantly change the post-vertical deprivation orientation 
perception of these observers, $R^{2}=.96, p=.02$ (Figure 23). All three subjects in the delay-in-darkness condition showed similar changes in orientation perception after the delay as they did without a delay.

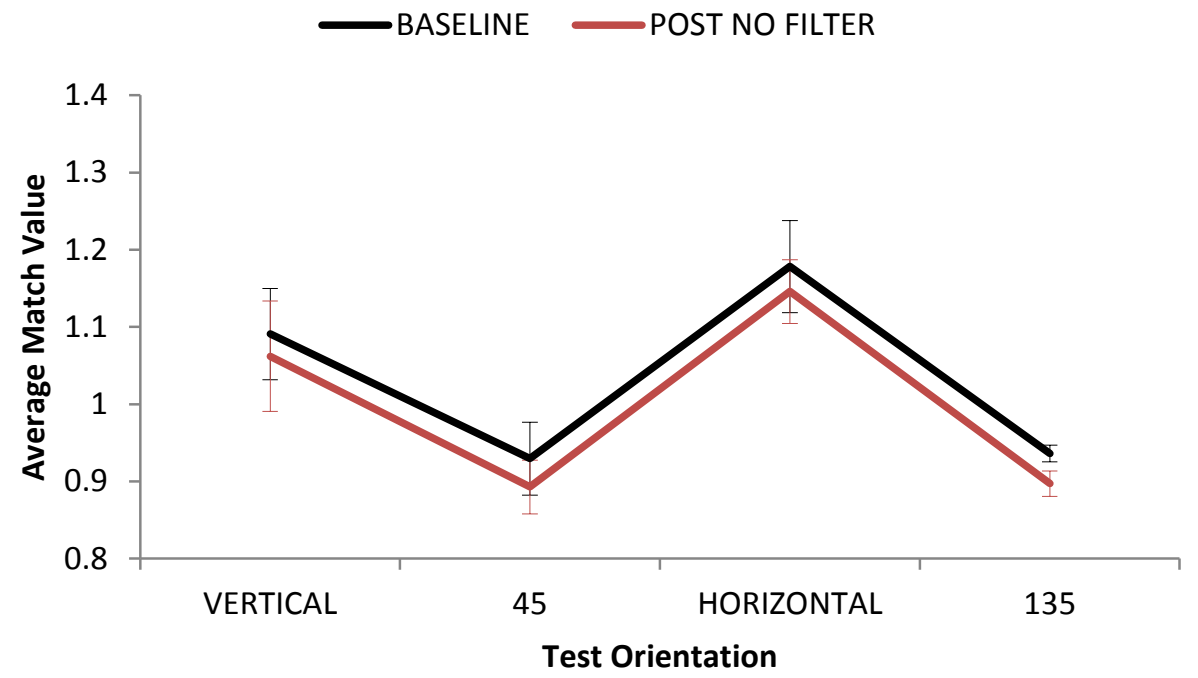

Figure 21. Subjects ( $\mathrm{n}=4)$ pre (black) and post (red) test match values after adapting to an environment with no filter (i.e. no induced change). Error bars represent standard error.

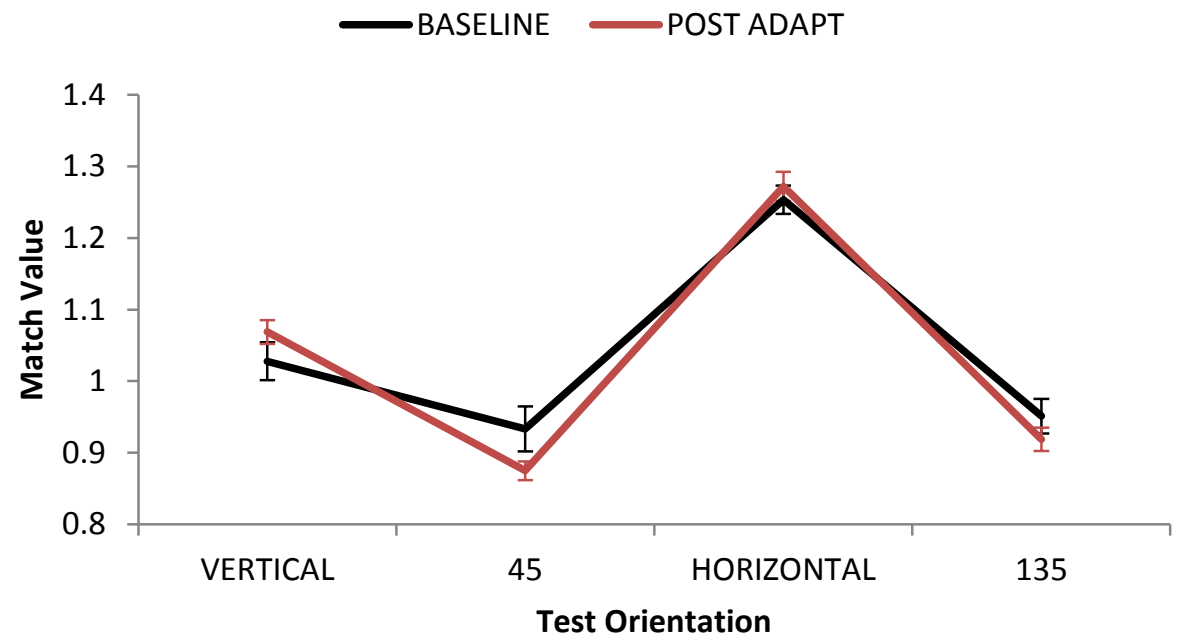

Figure 22. S2's pre (black) and post (red) test match values after adaptation to an environment wherein spatial frequency, but not orientation content had been altered. Error bars represent standard error. 
Conversely, S2 re-ran the same adaptation condition (vertical deprivation) and waited 30 minutes after adaptation to take the post-test. This time, however, S2 did not wait in darkness, but went about the day as usual (delay-in-light). As can be seen in Figure 24, this delay between adaptation and testing that included visual input caused S2 to return to baseline levels at the time of the post-test.

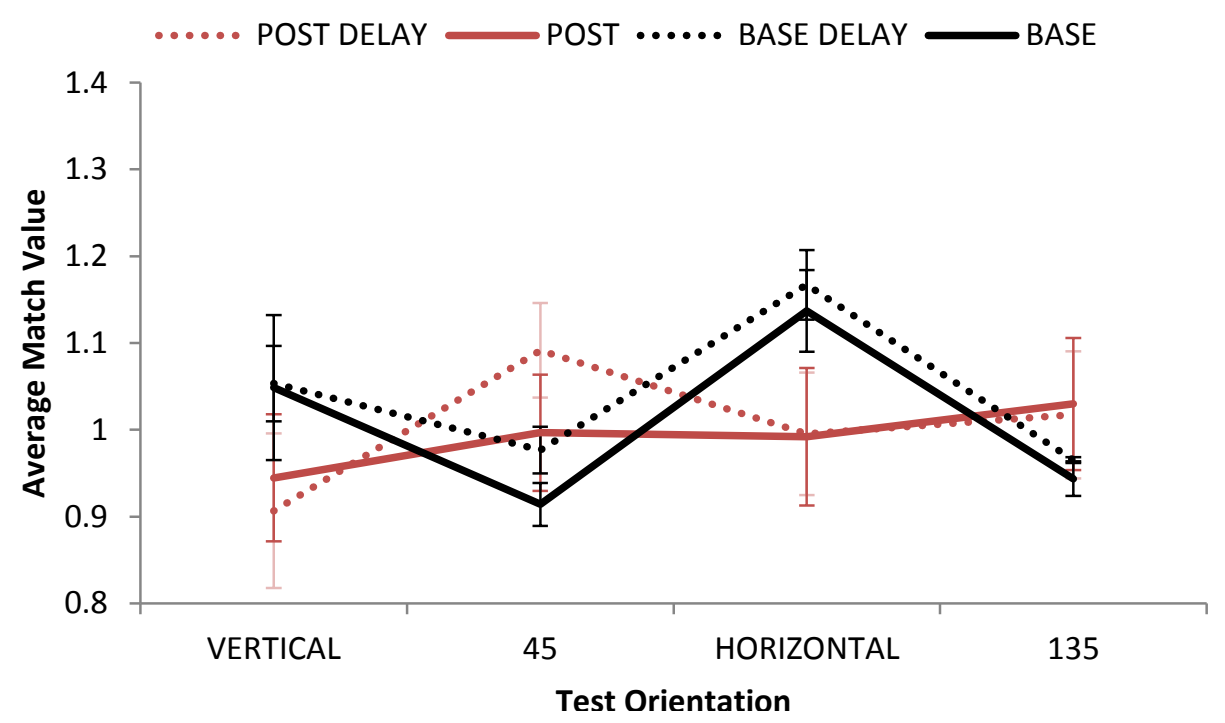

Figure 23. Subjects $(\mathrm{n}=3)$ pre (black) and post (red) test match values after adaptation to a vertical decrement with a 30 minute delay in darkness (dotted) or not (solid). Error bars represent standard error

Figure 23 also shows that subjects indeed changed their orientation perception after adaptation to vertical deprivation just as they did after horizontal deprivation (solid lines). As in the horizontal deprivation condition, sensitivity to both vertical and horizontal improved in the vertical decrement condition. Figure 26 shows that similar changes were exhibited by subjects adapting to oblique enhancement: subjects sensitivity to the cardinal orientations improved and sensitivity to the obliques worsened. To quantitatively compare across the three orientation conditions, effect sizes (Cohen's $d$ ) 


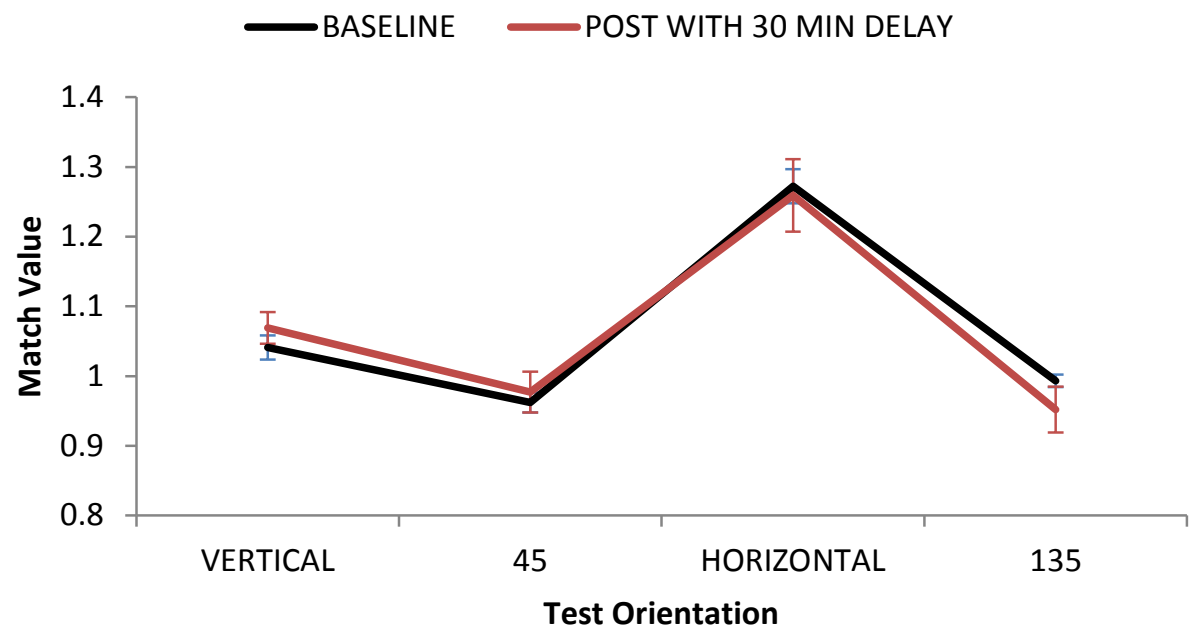

Figure 24. S2's pre and post-test matches after adaptation to vertical decrement with a 30 minute delay in light in between pre (black) and post (red) test. Error bars represent standard error.

were computed by taking the average difference between the pre and post test orientation match value of interest (horizontal, vertical, or the average of the two obliques) and dividing this by the pooled error rate (Table 4). That is, the effect size for the vertical deprivation condition represents the average change in vertical match value whereas the oblique condition represents the average (across $45^{\circ}$ and $135^{\circ}$ ) change in oblique match value. As Table 4 shows, the effect size for the vertical deprivation condition was substantially lower than for the horizontal deprivation or oblique enhancement conditions. This could be related to the lower KL divergence score in the vertical vs. horizontal deprivation condtion, although, if that were the case, one would expect that the oblique enhancement effect size would also be lower corresponding to it's lower level of KL divergence. The individual trial block data (Figure 26) from the vertical deprivation condition shows that not only do vertical and horizontal match values appear to be paired (as in Experiments 1-3), but also that vertical perception does notchange immediately 
following adaptation to vertical deprivation (although substantial perceptual change is still evident). Additional testing is necessary to determine exactly how vertical differs from other orientations and will be discussed further in the Follow Ups and General Discussion sections.

\section{Table 4.}

Effect size across different orientation adaptation conditions

Condition $\quad$ Effect size

Vertical Deprivation

1.119

KL Divergence

Horizontal Deprivation

2.87

Oblique Enhancement

2.48

0.0459

0.0518

0.0082

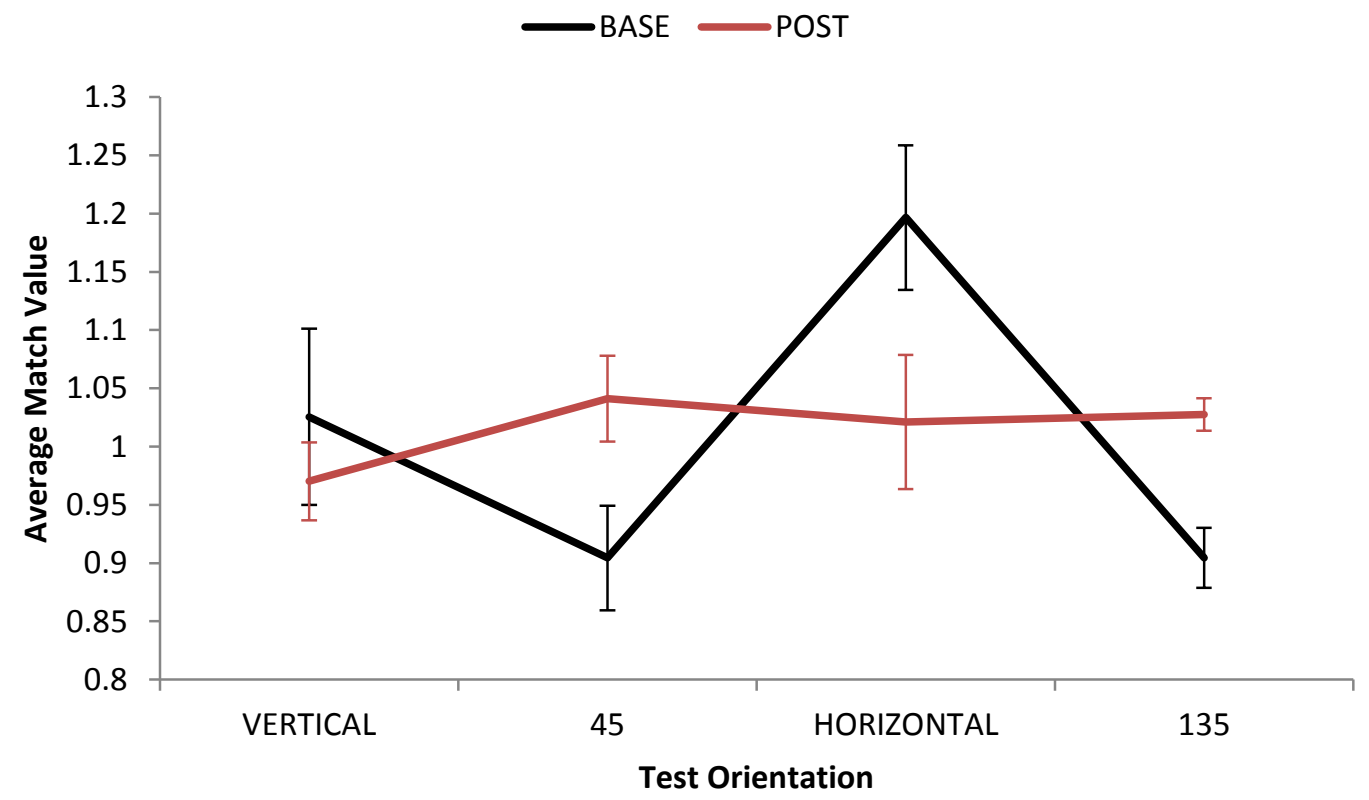

Figure 25. Subjects' pre (blue) and post (red) test match values after adaptation to an environment with oblique enhancement. Error bars represent standard error. 


\section{GENERAL DISCUSSION AND SUMMARY}

It is clear from the above experiments that changing the environmental statistics one is exposed to changes orientation perception in substantial and measurable ways. The duration of adaptation has an effect on the extent of perceptual change; the relationship appears to be best described as logarithmic, although it should be noted that the durations tested could favor this interpretation as the steps between duration conditions were not of equal length. There were substantial increases in all measures of perceptual change (horizontal effect, match value difference, effect size) as adaptation duration increased from 5 to 60 minutes. Unexpectedly, there was not much of an increase in the strength or duration of perceptual change beyond 2 hours of adaptation duration. Previous literature found that increasing adaptation from 2 to 4 and 4 to 8 hours significantly increased both the duration and magnitude of perceptual change (Bao \& Engel, 2012). The results above showed that adapting for 3 hours actually decreased the strength of perceptual change in comparison to adapting for 2 hours across all three measures (Table 1). This result was so surprising that 2 subjects (S2 and S3) actually reran the three hour adaptation condition, but again showed a smaller and shorter perceptual effect in the second run (Table 1 values include second run only, although results were similar). Previous experiments never actually adapted subjects for 3 hours 
(only 1, 2, 4, and 8) and the data in Table 1 do show an increase from 2 to 4 hours in the horizontal match difference score and both measures of the time to return to baseline (Bao \& Engel, 2012; Zhang et al., 2009). Therefore, these results are not in complete disagreement with what has been found previously. It could be that there are different timing mechanisms at work for adaptation durations shorter than 2 hours and longer than 4 hours. Further work breaking down the adaptation duration into shorter time periods would be required to investigate this hypothesis. Moreover, previous studies either did not mention the duration of post-adaptation testing (Zhang, et al., 2009) or only tested for a few minutes (Bao \& Engel, 2012; Haak, Fast, Bao, Leng, \& Engel, 2014). All of the post-adaptation effect measures in Experiment 1 were calculated using the first ten minutes of post-testing in order to average across at least four trials at any given test orientation. If it were possible to shorten the post-test period, more dramatic effects of adaptation duration may have been found. Another task would need to be implemented in order to obtain an accurate measure of orientation perception across different test orientations in such a short amount of time.

This study is the first of its kind to examine the relationship between the magnitude of physical change induced in the environment and the amount of perceptual change that it causes. Experiment 2 showed that a small physical change (35\% decrement) was not enough to change observers' post-adaptation orientation perception. On the other hand, larger increments of physical change (65\% and $85 \%)$ changed perception dramatically. As mentioned above, this is likely due to the overall change in the distribution of orientations as one moves from a fairly anisotropic, horizontally biased 
environment (original and 35\% decrement) to a more isotropic, vertically biased environment (65\% and $85 \%$ decrement, see Figure 9). While there was some indication of a logarithmic relationship between the physical and perceptual difference, this relationship was not significant. Given the similar lack of a significant relationship between these measures in Experiment 4, it seems more likely that Experiment 2 has determined the minimum physical change necessary to induce effects. While further experiments would be required to confirm this hypothesis, it appears that substantial changes in the orientation distribution will induced similar amounts of perceptual change while smaller changes will not be enough to alter the way the visual system biases perceptual information.

To enact the strong perceptual change that was seen in Experiment 1 after 1-2 hours, it seems that immersive experience is necessary. This finding is in concordance with virtual reality literature suggesting that immersive experience in the virtual environment leads to improved encoding and consequentially stronger perceptual effects (James, Humphrey, \& Goodale, 2001; James et al., 2002; Butler, James, \& James, 2011; Fredembach et al., 2009). Even the passive condition which did induce perceptual change (Passive 1) involved subjects viewing the altered environment over the HMD. Though the effects of adaptation were weaker after experience in this condition, there was still a change in orientation perception indicating that while real time feedback strengthens results, it is not necessary for perceptual change. 
Experience in an isotropic environment lessened or completely eliminated the horizontal effect anisotropy for all subjects. Most subjects show a more isotropic pattern after experience in an isotropic environment, and all subjects show less of a horizontal effect of orientation perception for the first five minutes of post-testing (Figures 16 and 17). The addition of the enhanced natural and oblique dominant adaptation conditions showed that the type of perceptual change induced by adaptation could be predicted by the distribution experienced during adaptation. When subjects experienced an environment in which the natural anisotropy had been inverted, their perceptual anisotropy was eliminated. When subjects experienced an environment in which the natural anisotropy was emphasized, the perceptual anisotropy was increased. Interestingly, the isotropic condition did not eliminate the anisotropy completely, but was the condition in which the horizontal effect measure was the lowest for the three subjects who ran all three conditions. Moreover, the oblique dominant condition did not invert the perceptual anisotropy, but eliminated it completely (Figure 18). Further theoretical discussion of this intriguing result is reserved for the Conclusions section.

From the control tests it was determined that simply viewing the environment in black and white or with a filter affecting the amplitude spectrum was not the cause of the altered orientation perception in Experiments 1-4. If the environment the subjects were exposed to in the lab were significantly different from that they were exposed to just before beginning the experiment, one would expect the baseline score to deviate from the unfiltered-HMD wearing control, but it did not. It can thus be concluded that the prefiltered environment was a good estimate of the 'typical' environment observers were 
exposed to before pre-test. The delay condition was of particular interest because it allows one to hypothesize about what causes the return to 'normal' orientation perception after adaptation. The delay-in-darkness control determined that the return to the normal anisotropy seen by the end of the post-testing period for most observers was not due to time alone. This condition, in addition to the delay-in-light control (run only on S2), showed that it must be the experience with typical visual input that caused observers to return to their usual orientation perception after adaptation.

While the control tests were planned before implementation of any of the experiments listed above, the data obtained yeiled unexpected results which raise further questions. Namely, how to characterize the relationship between vertical and horizontal orientations seen in Experiments 1-3 and the difference between horizontal and vertical susceptibility to adaptation demonstrated in the control experiments (Figures 7, 11, 13, and 26, Table 4). For these reasons, two follow-up tests were executed: one attempted to replicate some of the horizontal change across duration (Experiment 1) for vertical deprivation and the second sought to determine if the oblique orientations would exhibit a paired link like the cardinals. Due to the nature of these follow-ups as well as timing constraints, only two subjects were run in each condition. The exact parameters are detailed below. 


\section{FOLLOW UP TESTS}

\section{Methods}

Experiments 1-3 exhibited an unexpected relationship between the cardinal orientations: even though the peak of the decrement filter was concentrated at horizontal, sensitivity to vertical improved as well (see Figures 7, 11, 13 and 26). To examine this link in more detail, two sets of follow-up tests were run. The first set examined whether decrementing vertical orientations for different adaptation durations would produce a similar link between the cardinal orientations as was found in Experiment 1. Subjects 1 and 2 (both female, 1 naïve) adapted to 30 minutes, 1 hour, and 2 hours of vertical decrement with the same filtering parameters as the horizontal decrement condition from Experiment 1, but with the filter peak centered at vertical. The second set examined whether oblique orientations would be linked like cardinal orientations. As the control condition examined the amount of perceptual change as a result of enhancing both oblique orientations, this condition focused on decrementing only one of the oblique orientations, namely $45^{\circ}$. Again, the same filtering process was used in this condition as Experiment 1 with the filter centered at $45^{\circ}$ as opposed to horizontal. Subject 2 adapted to the $45^{\circ}$ decrement for 2 hours. 
Results

The results of the first set of follow-up adaptation conditions are plotted in Figure 28 along with each subject's horizontal decrement results (from Experiment 1) for direct comparison. There does appear to be some link between the cardinal orientations in both the horizontal decrement (Figure 26 right column) and the vertical decrement (left column) adaptation conditions. More subjects would be needed to completely elucidate the link between cardinal orientations.

A

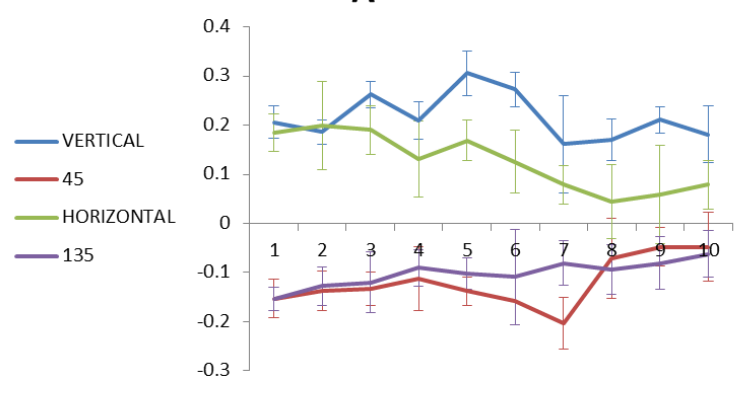

B

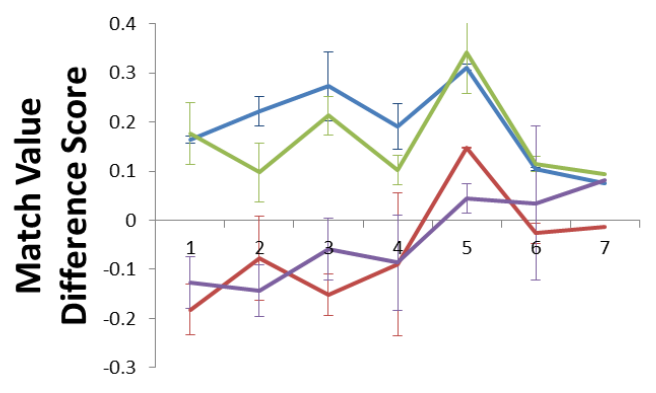

C

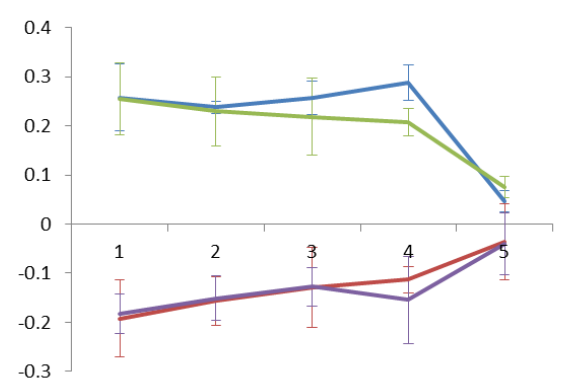

D

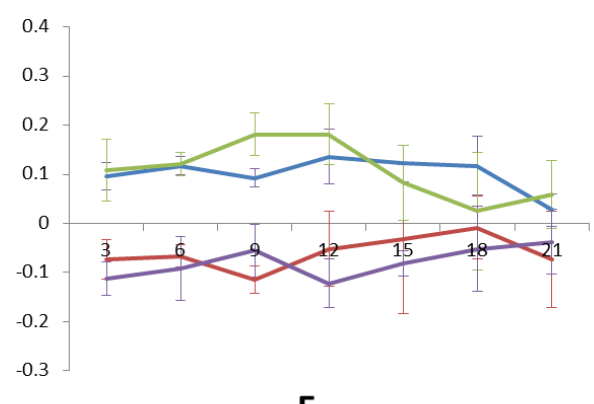

E

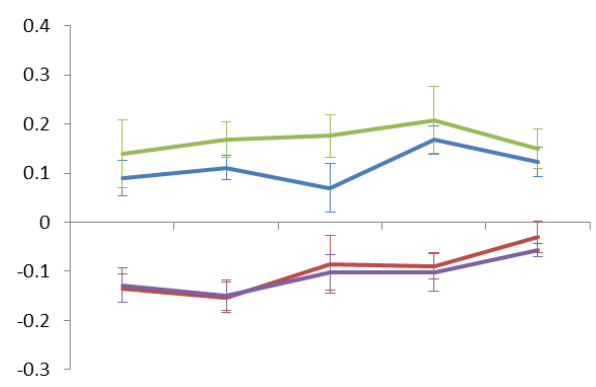

$\mathbf{F}$

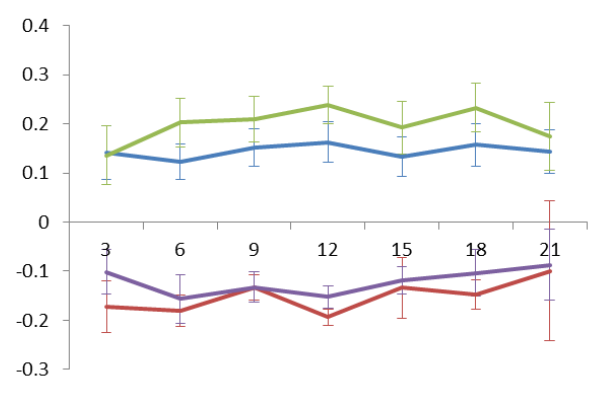

Post-adaptation Trial Block (average of 3)

Figure 26. Post-adaptation difference scores for S1 and S2 adaptation to vertical (right column: A-C) and horizontal (left column: D-F) decrements across three adaptation durations: 30 minutes (A and D), 1 hour (B and E), 2 hours (C and F). 
The second set of follow-ups are plotted in Figure 27: the post-adaptation match value difference scores for both obliques appear to be linked just as the cardinal orientations were linked in the horizontal decrement condition. That is, even though the adaptation paradigm decremented only the $45^{\circ}$ oblique, sensitivity to the $135^{\circ}$ oblique also improved.

A
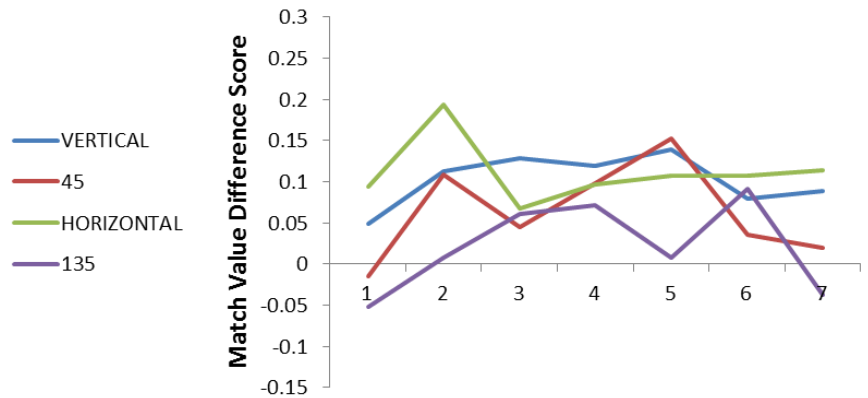

Post Adaptation Trial Block ( Average of 3)
B

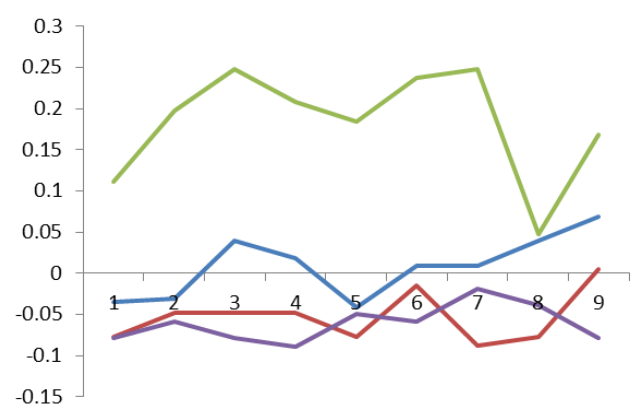

Figure 27. Post-adaptation difference scores for $\mathrm{S} 2$ adaptation to $45^{\circ}$ (A) and horizontal (B) decrements after 2 hours. B has been re-plotted from Experiment 1 for this subject. 


\section{MODELING}

As stated above, the perceptual results of each type of adaptation manipulation can be predicted by the changes imposed on the amplitude spectrum. Explicitly, the distribution of orientations experienced during adaptation is an inverse of the perceptual salience of different orientations. The use of a Bayesian encoder-decoder model allows for the modeling of the perceptual results while taking into account the environmental experience of each observer. As discussed in the introduction, perception is biased by both the likelihood of stimuli in the environment as well as their prior probability, and previous research has shown that human observers' priors are a good match to the environmental distribution (Stocker \& Simoncelli, 2006a; Girshick, Landy, \& Simoncelli, 2011). That is to say, observers' priors are biased in terms of orientation which leads to perceptual biases across orientation, in this case, the horizontal effect. That is, the match between the environmental distribution and observers' priors leads to the anisotropic suppression of cardinal orientations which is presumed to cause the horizontal effect (Essock, Haun, \& Kim, 2009). If one assumes, then, that observers are using something like the natural distribution of orientations as their prior during the pre-test, one can model the change in both the likelihood and the prior distribution across adaptation conditions. These observers show a horizontal effect pattern of results on the pre-test matching experiment indicating that they used the natural environmental prior as would 
be expected. Assuming that their prior probability distribution changes based on the statistics of the adapted environment, the post-test should show different results that can be predicted using the experimentally obtained data.

\section{Results}

We assume that observers use the correct likelihood function which can be obtained from interpreting the measurement noise distribution as a function of the stimulus for a particular measurement (Girshick, Landy, \& Simoncelli, 2011). The measurement noise distribution describes the probability of a sensory measurement given a particular stimulus value (Girshick, Landy, \& Simoncelli, 2011). The variance of the perceived/estimated value, as measured experimentally, is used to estimate the width of the measurement distribution across orientation (Stocker \& Simoncelli, 2006a). That is, to estimate this measurement noise across all orientations, the standard error obtained in the matching experiment before and after adaptation at each test orientation was interpolated using cubic spline in Matlab (Stocker \& Simoncelli, 2006a; Girshick, Landy, \& Simoncelli, 2011).

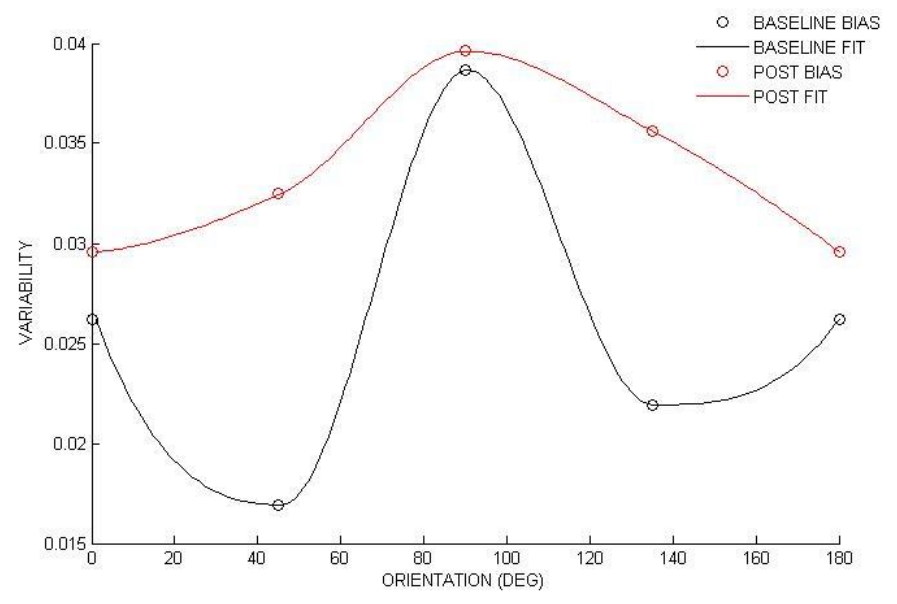

Figure 28. Variability across orientation pre (black) and post (red) adaptation to an isotropic environment. 
For the particular biases show in Figure 28, subjects were adapted to an isotropic environment (Experiment 4). As can be seen, the variability in perceived strength of orientation changed after adaptation such that subjects were more variable across all orientations, but especially at the obliques. Although not depicted in every figure, the same model was also applied to data from the enhanced natural and oblique dominant conditions. The interpolated variability data were then used to compute the width of the measurement distributions across orientation using a Von Mises function that peaks every 180 degrees, $\mathrm{P}(m \mid \theta) \approx \mathrm{e}^{K \cos (2(\theta-m))}$ where $K$ is determined by the interpolated data, $m$ ranges across orientation from $0-180^{\circ}$, and $\theta$ determines the peak of the Von Mises and is centered at the measured orientation. The Von Mises distributions are calculated for each stimulus value across the stimulus space $\left(0-180^{\circ}\right)$. The resulting $2 \mathrm{D}$ matrix consists of columns of measurement distributions (probability of a measurement given a particular value) and rows of likelihood distributions (probability of a stimulus value given a particular measurement) (Figure 29 top row; Girshick, Landy, \& Simoncelli, 2011). Taking horizontal slices, the likelihood as a function of orientation then looks like the bottom row of Figure 29 (pre-adaptation left, post-adaptation right). The likelihood functions are slightly asymmetric because the measurement distribution width varies across orientation. Moreover, as one can see, for the isotropic adaptation condition, the likelihood distributions went from being extremely anisotropic, to more isotopic overall. This corresponds to the more isotropic pattern of orientation perception seen after adaptation in Experiment 4. Similar relationships were seen in the data from the enhanced natural and oblique dominant conditions (not depicted). 

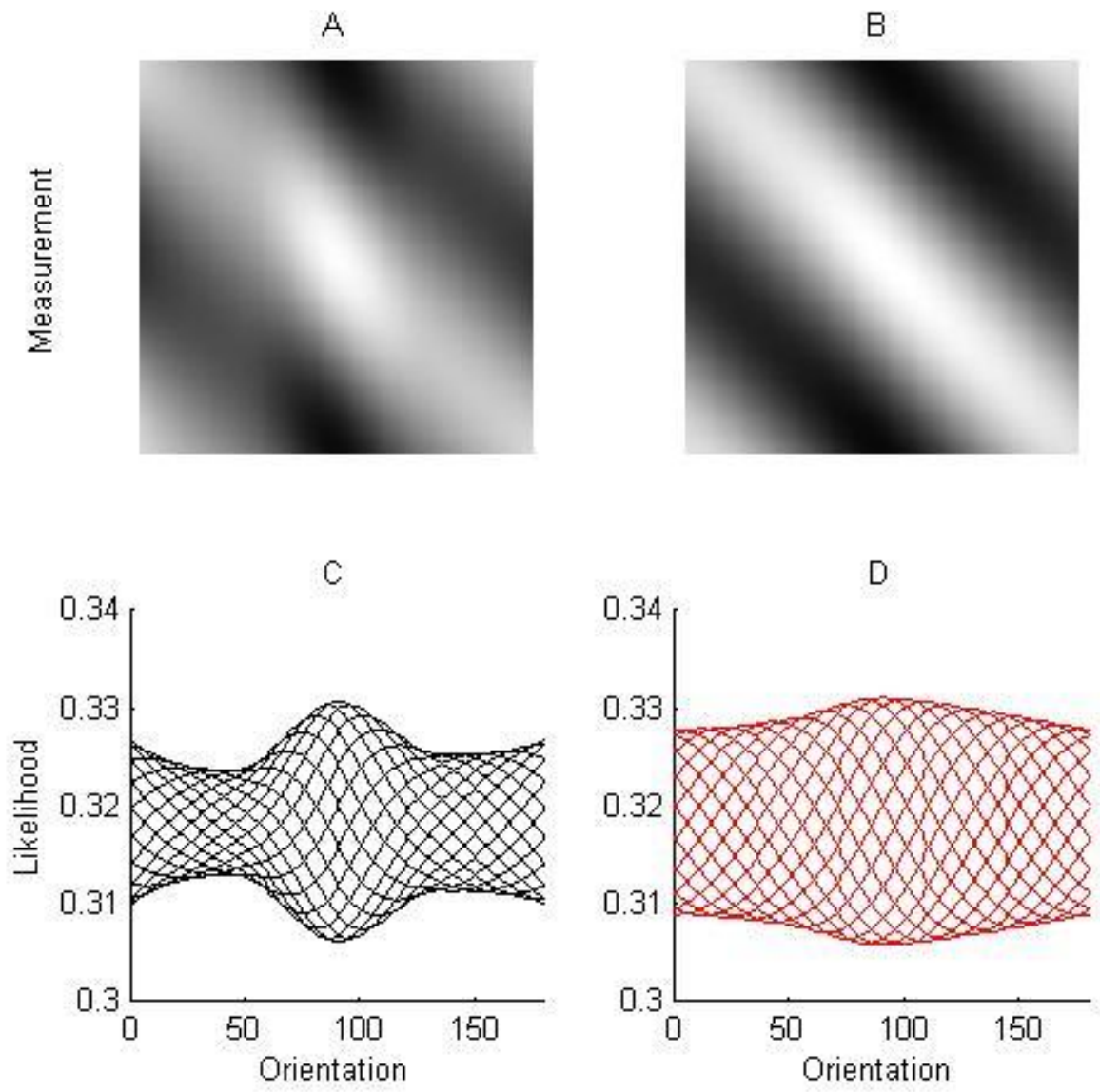

Figure 29. Likelihood and measurement distributions pre (left) and post (right) adaptation to an isotropic environment.

To obtain an estimate of the pre-test prior in all conditions, we used the orientation spectra of over 300 natural images obtained by taking photographs in and around Kentucky and Indiana. The post-test prior was calculated by computing the orientation spectra of the filtered images sampled from the video recordings made during adaptation for each condition. The spectra were calculated using the methodology detailed in our previous report (Schweinhart \& Essock 2013). Briefly, each image is rotated every three degrees and a discrete fast Fourier transform is performed. The 
amplitude spectra is then convolved with a filter centered at horizontal and vertical with a three degree orientation bandwidth to extract the amplitude of those specific orientations. As this process is repeated every three degrees, the entire spectra can be calculated across orientation. However, as mentioned above, the prior should not be an exact match to the natural world, but rather a 'regularized' match in order to compensate for noisy environments (Feldman, 2013). Therefore, the fit of both priors was estimated using spline interpolation at 5 points so as not to 'over-tune, $\log (p(\theta))=a_{i}\left(\theta-\theta_{i}\right)^{3}+b_{i}(\theta-$ $\left.\theta_{i}\right)^{2}+c_{i}\left(\theta-\theta_{i}\right)+d_{i,} \theta_{i}=\left[0^{\circ}, 45^{\circ}, 90^{\circ}, 135^{\circ}, 180^{\circ}\right]$.

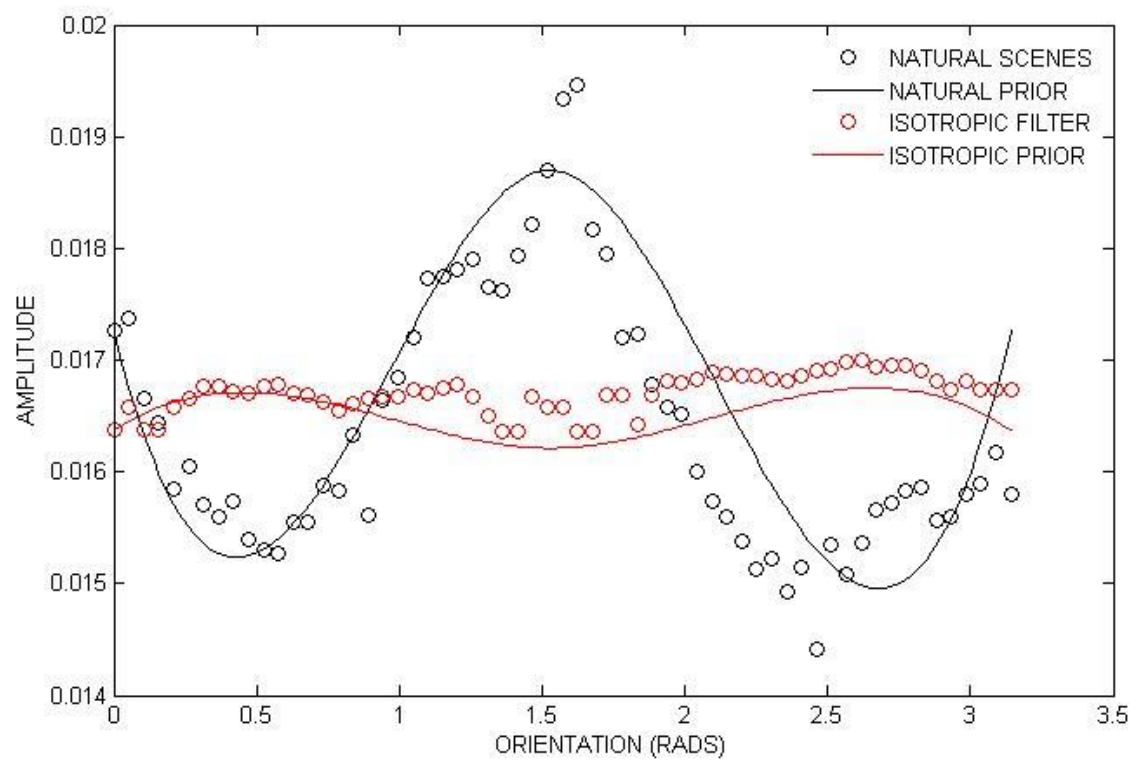

Figure 30. Sample of every three degrees (dots) from natural scenes (black) and the filtered images used in the isotropic adaptation condition (red). Lines represent the interpolated fits used for the model.

Each of the experiments discussed above measures observer's orientation perception by having subjects match naturalistic, oriented, noise stimuli at different orientations to a standard in terms of perceived salience. The observer's match values 
(test scalar/reference scalar) serve as a representation of his/her particular bias at the tested orientation: the difference in physical strength of two oriented patterns which are perceived to be equal (Girshick, Landy, \& Simoncelli, 2011). Bias is approximately equal to the product of the squared likelihood width (variance) and the slope of the log of the prior (Stocker \& Simoncelli, 2006a; Girshick, Landy, \& Simoncelli, 2011). Using the full width (in degrees) of the modeled likelihood distributions at half height (FWHH) and the slope of the log of the prior (before and after adaptation) at the same five points the bias was thus estimated, FWHH $* \frac{\log (p(\theta i))-\log (p(\theta i+1))}{\theta i-\theta i+1}$. The predicted bias values for these control points are then used to interpolate (cubic spline) across the range of orientations and compared to the bias obtained experimentally both before and after adaptation in an enhanced natural, isotropic, and oblique dominant environment (Figure $31 \mathrm{~A}, \mathrm{~B}$, and $\mathrm{C}$ respectively). Figure 31 shows that the interpolated model data (solid lines with standard error area shaded) provide a good fit to the experimentally obtained bias (solid points with standard error bars) across each adaptation condition. Whereas experience in the isotropic environment eliminated the horizontal effect while experience in an environment filtered to have an increased cardinal bias led to a slightly greater perceptual anisotropy. Lastly, experience in an environment with a reversed anisotropy (oblique dominant) produced a diminished horizontal bias, although it did not completely reverse the perceptual bias. All changes are well predicted by a model that assumes participants update their prior based on recent experience. 
Via trial and error, it was determined that altering the temperature of the prior distribution (by raising it to an exponential power) provided better fits to the experimental data. That is, the basic shape of the distribution remains the same, but it is stretched or flattened to better fit the data. The exponent for each prior that best fit the data was determined using least squared error. The experimentally obtained match values (shifted by -.9) at each tested orientation were then compared to the interpolated values at these same orientations for each subject to evaluate the fit of the model in the isotropic condition. In other words, the repeated measure ANOVA from Experiment 4 was re-run with the added factor of model $\mathrm{x}$ data. The main effect of this model factor was not significant indicating that the model points were not significantly different than the experimentally obtained data, $F(1,8)=.65, p=.44$.

The alterations to the environment experienced during adaptation in Experiment 4 represent globally altered environmental distributions whereas previous accounts only tested changes in specific orientations (Bao \& Engel, 2012; Girshick, Landy, \& Simoncelli, 2011; Zhang et al., 2009) Therefore, while previous reports have argued for changes only in the likelihood distribution, this manipulation of the global distribution of orientations enables testing the malleability of the prior as well (Stocker \& Simoncelli, 2006b). In response to experience in such globally altered environments, local changes in likelihood functions only in the vicinity of adaptor values may be inadequate to efficiently encode experience. To evaluate whether subjects were indeed changing their likelihood and prior probability distributions, the model of post-adaptation perceptual biases was applied using both the natural orientation distribution and the isotropic 
A
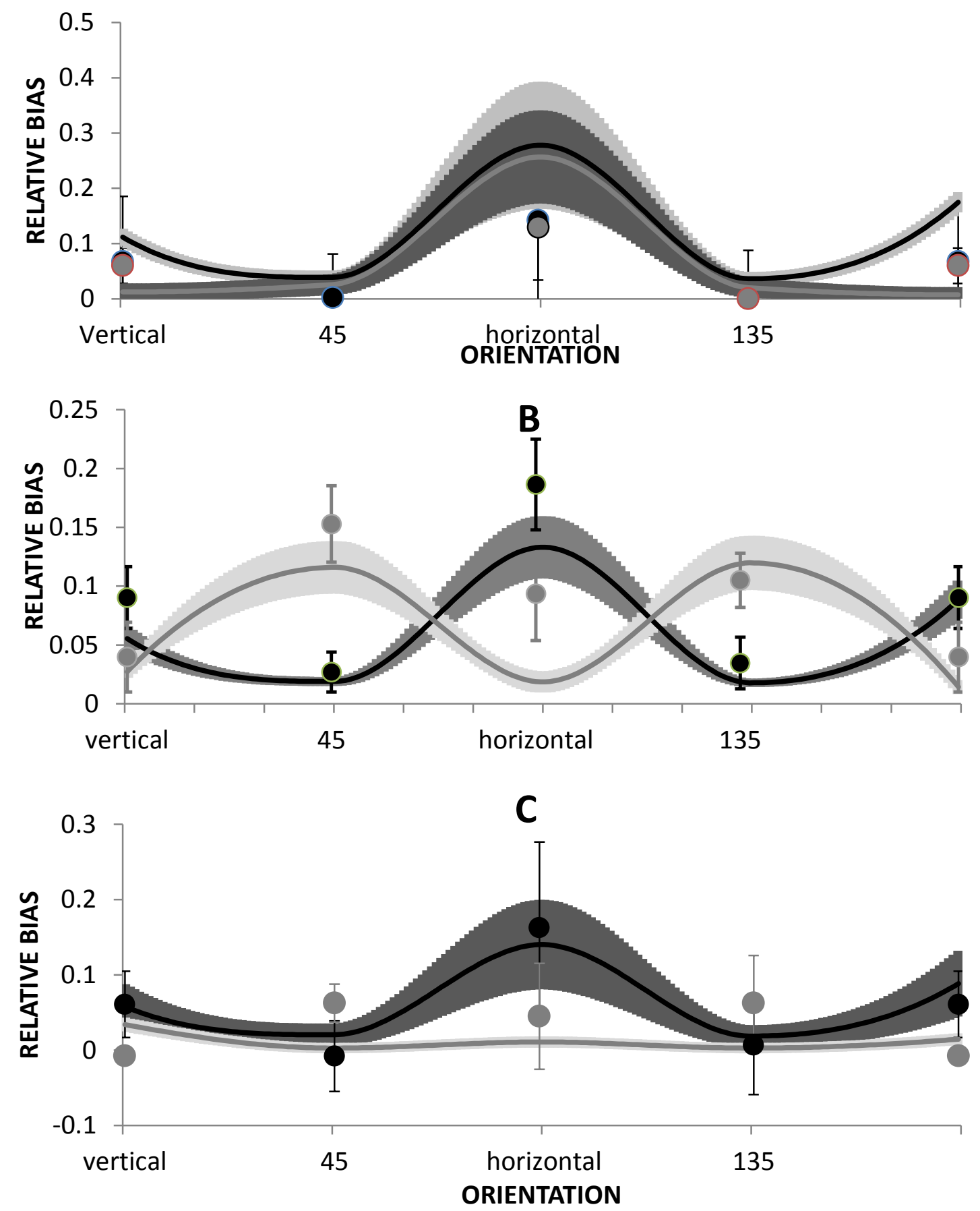

Figure 31. Experimentally obtained bias (points) and model fits (lines) for each of the globally altered distributions: enhanced natural (A), isotropic (B), and oblique dominant $(\mathrm{C})$. For each plot, the black line and points are the pre-adaptation data and the grey line and points are the post-adaptation data. Error bars on the points and shaded regions around the modeled lines represent standard error. 
orientation distribution as a representation of the prior for the post-test biases. As seen in Figure 32, the model using the isotropic prior (lighter grey) provides a better (by eye) fit to the data than one that uses the post-adaptation likelihood, but the natural distribution of orientations as the prior (darker grey). The difference between the post-adaptation data and each model was compared using a 2 (model: natural, isotropic) x 4 (orientation: 0 , $45,90,135)$ repeated measures ANOVA. A significant interaction indicated that the models differed in their fit to human perceptual biases after isotropic experience as a function of orientation, $F(4,32)=24.44, p<.001$ (see Figure 32). Thus, perceptual biases are best explained by a prior that reflects the environmental statistics of recent experience.

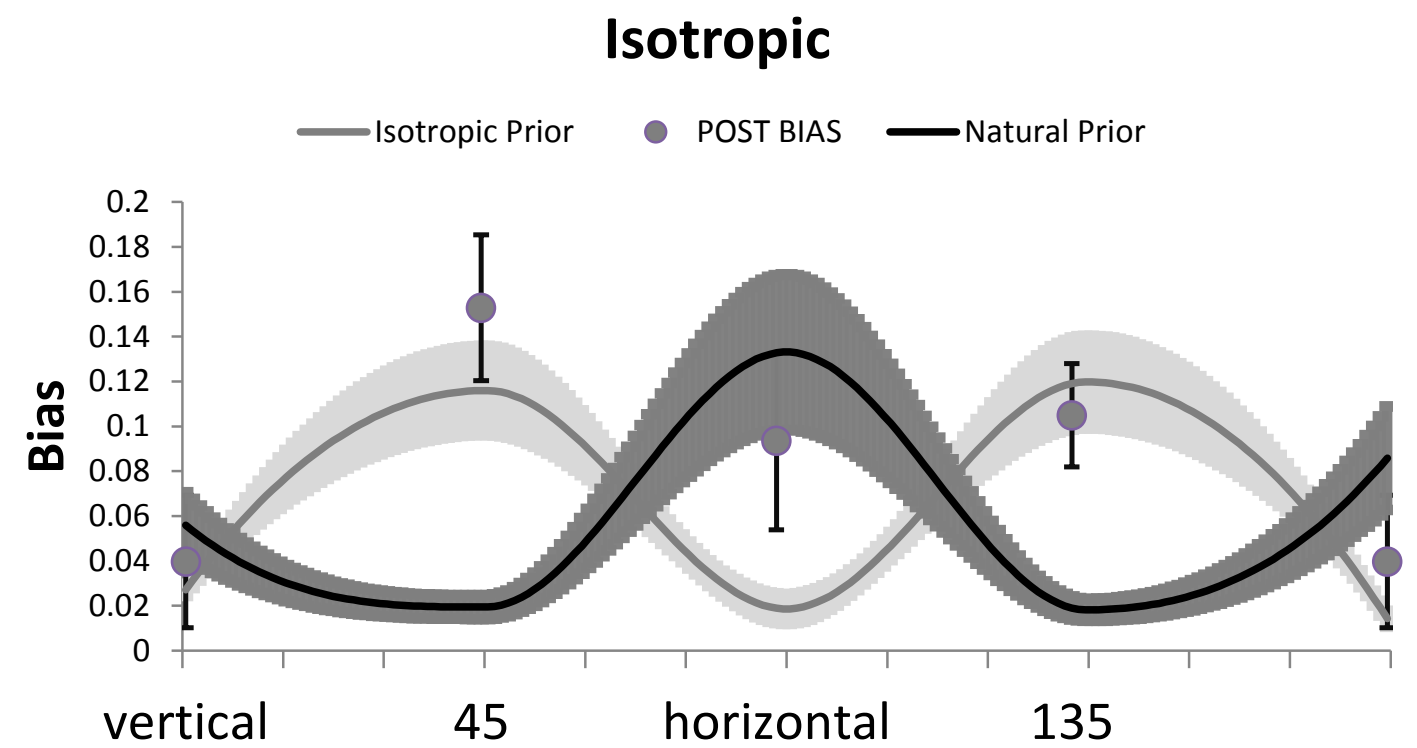

Figure 32. Experimentally obtained post-adaptation bias from the isotropic condition is modeled using both the isotropic distribution as the prior (grey) and the natural distribution as the prior (black). Error bars on the points and shaded regions around the lines represent standard error. 


\section{CONCLUSIONS}

These data indicate that broadband orientation perception (and subsequently the horizontal effect) is in fact susceptible to the effects of adaptation. Effects were observed with as little as 30 minutes of experience with altered environmental orientation content, but these effects are stronger and longer lasting with longer experience, at least up to two hours (Experiment 1). Moreover, the amount of perceptual change is somewhat dependent on the extent of physical change, although the exact relationship remains to be determined (Experiment 2). Adaptation in an immersive altered reality also appears to induce stronger effects than more passive adaptation, indicating that real-time visual feedback strengthens perceptual change (Experiment 3). Lastly, global changes in the orientation distribution experienced by observers have predictable effects on the pattern of orientation biases exhibited post-adaptation implying a relationship between the statistics of our visual environment and our perception of structural content. That is, changing the amplitude of the structural content that we see has a dramatic effect on how we perceive subsequently presented structure in a naturalistic background.

As mentioned in the introduction, this experiment is not the first time that such long-term, environmental changes have been recorded; long-term adaptation (4 hours) to decreased environmental contrast increases subject contrast sensitivity (Kwon et al., 2009). This increase in sensitivity was presumed to occur at the cortical level as effects 
were transferrable between the eyes (Kwon et al., 2009). Kwon and colleagues (2009) contend that whereas typical shorter-term adaptation results can be explained via a shifting in the response function towards the adapting value (what they call contrast gain), longer-term adaptation to reduced contrast involves a steepening of the contrast response function near the mean level (response gain). That is, whereas short-term adaptation maintains the shape of the response function while changing the mean, longterm adaptation changes the shape but retains the mean. Although these results are discussed in the terms of contrast response, they can be applied to the current results as well. Like decreasing the overall contrast signal for a long period of time, the current studies investigated the effects of decreasing horizontal orientations for an extended period. It seems necessary, then, that the current results would be best explained by changes to a longer-term adaptation mechanism, one that changes the shape of the orientation distribution.

Perhaps even more relevant to the current results, long-term adaptation has also been studied using similar orientation decrements across different adaptation durations, but with a narrowband orientation perception task (TAE: see Introduction, Bao \& Engel, 2012; Bao, Fast, Mesik, \& Engel, 2013; Haak et al., 2014; Zhang et al., 2009). These studies have provided evidence for two distinct processes explaining perceptual effects after short and long-term adaptation. The strongest evidence for the two mechanism theory has come from studies wherein de-adapting and re-adapting to previously adapted stimuli show spontaneous recovery of adaptation effects (Bao et al., 2013). That is, once subjects were no longer exhibiting perceptual effects of long-term adaptation, such 
effects could be reinstated much more quickly than before (after only a few minutes of experience; see Introduction and Bao et al., 2013). Presumably, the results reported here are also effects of adapting a long-term, cortical mechanism and should thus show spontaneous recovery as well as intraocular transfer if tested in the future.

Similar studies have also determined that long-term adaptation effects follow a 'duration scaling' law wherein the effects of adaptation get stronger and longer lasting as the adaptation duration lengthens (Bao \& Engel, 2012; Bao et al., 2013). As revealed in Experiment 1, the relationship between duration of adaptation and magnitude of perceptual effects found here is not linear; while effects increased monotonically from 560 minutes of adaptation, the perceptual change began to plateau after 2 hours. The difference in perceptual effect seen between these time frames could be due to what Engel and colleagues (2014) call the "cost of adaptation" (Haak et al., 2014). In previous studies, altered environmental experience over 4 days of continuous adaptation caused a peak effect of perceptual change early on in experimentation (i.e., the first or second day) that decreased after more experience (Haak et al., 2014). The authors explain the maximum effect on the first day as the action of a 'fast' adaptation process while later continued (although weaker) effects were attributed to a slower process. The fast process showed an initial decline because the metabolic and coding costs of remaining adapted outweighed the perceptual benefits (Haak et al., 2014). While the subjects in these experiments were not adapted for nearly so long a time, it could be that the results of the experiments listed above are due to differences in long/short-term and fast/slow mechanisms of adaptation. Assuming these results are due to adapting a long-term 
mechanism, it could be that they follow an initial duration scaling due to the 'fast' acting mechanism for the first two hours. The plateau of effects seen in the three and four hour adaptation conditions could be due to the decay of fast mechanism before the slower mechanism can take effect.

Though these time scales do not align perfectly with the days tested previously, there are many differences between the two tasks that could explain these variations (Haak et al., 2014). The task used here (broadband, suprathreshold matching) is very different than those that have been used in the past to measure the effects of adaptation (e.g., narrowband contrast discrimination of orientations). The initial peak, presumably due to the fast acting mechanism, also differs slightly; whereas these subjects show a decline in perceptual change after 3 hours, subjects tested on contrast discrimination exhibited a decline on the first day as a sign of the faster mechanism (although the exact timing of the first testing day is not discussed; Haak et al., 2014). These two mechanisms can also be explained in the terms of a Bayesian framework; longer experience in an environment produces greater confidence in the pattern of observed structure and would thus lead to stronger and longer lasting effects which would stabilize over time (Haak et al., 2014). Interestingly, recent work has indicated that adaptation to a previously experienced statistical ensemble is not faster than adaptation to a new ensemble (Mesik, Patke, \& Engel, 2015). That is, the advantage that adaptation provides is not retained in any meaningful sense once the adapted state is abolished. Indeed, the prolonged time it takes subjects to return to baseline levels in these experiments indicates that the typical perceptual whitening imposed by being continually adapted to natural scene statistics is 
not retained. However, these results are at odds with the findings that re-adapting subjects to previously experienced environments can reinstate adaptation effects more quickly (Bao et al., 2013). It will be interesting in the future to more explicitly examine the relationship between fast and slow adaptation and determine how long after deadaptation re-adaptation can be implemented to recover sensory advantaged gained during adaptation.

Until this experiment, all previous studies of immersive adaptation had focused only on either incrementing or decrementing specific orientations (Bao \& Engel, 2012; Bao et al., 2013; Haak et al., 2014; Zhang et al., 2009). Experiment 4 is of particular interest as the physical change implemented did not concentrate on only one band of orientations, but rather effected the global distribution of all oriented structure in the image. Therefore, this experiment was key in determining how the environmental structure affects perceptual biases. The results indicate that observers have an accurate representation of the regularities present in their environment that informs their perception of features in context. While the 'typical' distribution leads to the anisotropic bias of the horizontal effect, altering the entire orientation distribution of the environment causes predictable changes in orientation perception. Immediately after adaptation, subjects appear to inversely match their perceptual biases exactly as expected given the experienced altered environment (Figure 20). However, these initial effects decay quickly, especially for the Enhanced Natural environment. Additionally, the average effect of an Oblique Dominant environment was not to invert the typical horizontal effect, but rather completely eliminate any bias across orientation (Figure 18) perhaps 
due to variability in the data or the limit of adaptation (see below). The effects of the Isotropic and Oblique Dominant condition seem to last longer, perhaps indicating that a larger deviation from the expected distribution of orientations produces greater perceptual change. Further testing would be necessary to determine how precisely perceptual change matches the experienced environment and the duration of the effects of global environmental change.

One potential confound to the current results would be the difference between changing the energy of the global image (as implemented here) and changing the energy of local structural components of the image (Hansen \& Hess, 2007). While the filters applied in Experiments 1-3 and the global changes of Experiment 4 change the average orientation content across the entire image, the phase spectra (containing structural relations; Morgan, Ross, \& Hayes, 1991; Oppenheim \& Lim, 1981; Piotrowski \& Campbell, 1982; Shapley et al., 1990) of all images remained intact. It is possible, then, that conflicts between the global and local image statistics could exist. That is, while the amplitude spectrum of a given image in Experiment 4, for example, would be isotropic, the alignment of particular structures in the phase spectrum could lead to local areas of the image that were not isotropic. However, in cases when the global and local statistics were in conflict, one would not expect to see the obtained results of adaptation to the global image statistics. Specifically, if subjects were influenced by residual local image statistics differences, then adaptation to global changes should be more variable and not predictable given the orientation distribution to which subjects were exposed. Moreover, the local and global statistics of a particular image tend to be correlated as the local 
alignment of orientations is often what determines the global anisotropy. As supporting evidence, a control analysis was run on 417 natural scenes collected for use in other studies (e.g. Schweinhart \& Essock, 2013). The global statistics were determined in the same manner as the videos from the above experiments. The local statistics were calculated using a three part algorithm developed by Hansen and colleagues which uses a bank of filters (centered at 5 spatial frequencies and 12 orientations) to isolate predominate contours in the image (see Hansen \& Hess, 2007). After the analysis, only 30 of the images were found to have local and global image statics in conflict naturally. Furthermore, after filtering these 30 to have isotropic global orientation spectra (as in Experiment 4), only 4 retained a local anisotropy that was greater than one standard deviation above the mean for the entire image set. Therefore, it would seem that any effect of local image statistics would be negligible.

The lack of a complete inversion of anisotropy in the Oblique Dominant condition could be indicative of a limit to the plasticity of orientation perception. That is, it may be that subjects can change their perceptual biases based on environmental input, but perhaps only to a certain extent. Indeed, the data suggest such a possibility; while the immediate ( $\sim 5$ minutes) effects of inverting the naturally occurring anisotropy suggested perceptual inversion (Figure 21), the average post-adaptation effects were not discernable from the effects of isotropy (Figure 19). Furthermore, the changes in variability (as plotted in Figure 28) used to model the likelihood distributions were also anisotropic suggesting that while observers' estimation of oblique orientations were changed dramatically, the cardinal orientations were more stable. As discussed in the 
introduction, there is a predominance of cardinally tuned cells in early visual cortex -whose responses are more narrowly tuned and indeed more stable than the obliques - but this predominance can be overcome by differential suppression of orientation tuned responses in broadband contexts (Dragoi, Turcur, \& Sur, 2001; Essock, Haun, \& Kim, 2009; Hansen \& Essock, 2004; Haun, Hansen, \& Essock, 2006; Haun \& Essock, 2010; Kim, Haun, \& Essock, 2010; see also Schwartz \& Simoncelli, 2001; Wainwright, 1999). The perceptual anisotropy of the horizontal effect can be attributed to increased suppression of horizontally and vertically tuned cells (Essock, Haun, \& Kim, 2009; Haun \& Essock, 2010). When oriented structure is presented in a broadband context (as when targets are presented in the presence of masks), a masking component suppresses horizontal targets most and suppresses oblique targets least (Essock, Haun, \& Kim, 2009; Haun \& Essock, 2010; Hansen et al., under review). The effects of altering the global distribution of orientations could be explained by altering this component; whereas it typically weights horizontal the most (Essock, Haun, \& Kim, 2009; Haun \& Essock, 2010), adaptation in an altered environment could change the pattern of suppression across orientation. The combination of this adaptive component and the known cortical anisotropies could be a way to explain the limits of perceptual change. While the adaptive constant could change its suppressive weights, the differential tuning preferences of the cell population cannot change. Further research will be necessary to identify the limitations in the malleability of visual orientation perception.

Another unexpected result of the current study was the relationship between the cardinal and oblique orientations as affected by adaptation; change in the environmental 
distribution of one orientation caused perception of the orthogonal orientation to change as well. As demonstrated by the follow-up experiments, decrementing horizontal only, vertical only, or only one oblique caused sensitivity to increase at the deprived orientation as well as the orthogonal orientation (Figures 28 and 29). Such orthogonal facilitation effects have been found using contrast matching experiments, but in those tasks orthogonal orientations were presented in the surround and effects were very small (Xing \& Heeger, 2001; Yu, Klein, \& Levi, 2001). Many studies have demonstrated such an effect of cross orientation facilitation and have attributed this to a release from inhibition (e.g., Chen \& Tyler, 2002). Orientation-tuned cells in visual cortex inhibit other cells tuned to similar orientations. However, during visual deprivation, when the visual system is deprived of a specific orientation, the inhibition between neighboring cells is lessened. That is, normally the typical inhibition between the orthogonal orientations is decreased during adaptation (when one orientation is decremented) and thus sensitivity to both orientations increases after adaptation. However, the effects seen here cannot be attributed to release from inhibition alone as it would not explain the decrease in sensitivity to orientations $45^{\circ}$ away from the adaptor. Release from inhibition would predict greater increases in sensitivity to orientations nearer the adaptor (as this is where inhibition is greatest), whereas these data show decreases in sensitivity to orientations other than the adaptor and the orthogonal (e.g., Figure 7). Other studies have suggested that orthogonal orientations increase contour detection and therefore may have longrange faciliatory interactions (Ledgeway, Hess \& Giesler, 2003). However, while these connections may be necessary for contour integration, their existence is speculative and 
would not necessarily bear on the current findings. Therefore, the cortical locus for the relationship between the orthogonal orientations revealed here is entirely unknown and must be left for future investigations.

One way to begin determining where in the brain the adaptive changes to environmental statistics may be occurring is to determine how long it takes effects to emerge. Adaptive changes that occur at early levels of the visual hierarchy (i.e., V1) should occur relatively quickly whereas changes which take longer to appear may be due to feedback mechanisms higher up in cortex. It would be of note, then, to model the changes that occur as an effect of increasing the duration of adaptation in Experiment 1. As discussed, the perceptual magnitude changes are not linear with increasing duration of adaptation, perhaps due to differences in fast and slow adaptation mechanisms. Applying the model developed for global changes in the orientation spectra to these conditions, in which only local changes were made, could determine the amount of adaptation experience necessary to cause a shift in the prior distribution. Whether the prior can change rapidly or takes much longer to completely match the newly experienced environment could then indicate whether the change in happening at low or high levels cortically.

One problem with modeling the data from Experiment 1 is that the changes made to the orientation distribution, while local, necessarily cause reciprocal changes in the global distribution; decrementing horizontal necessarily increases the relative proportion of vertical. These reciprocal changes would predict perceptual changes that are not found 
in the experimental data -- decrementing horizontal actually increased sensitivity to vertical rather than decreasing it. The intriguing relationship between the orthogonal orientations could also be built into the model to replicate the experimental findings. If it is assumed that the orthogonal orientations must be related, it could be further assumed that there are two separate mechanisms at work in determining the probability of specific orientations in the environment: one for the cardinals and one for the obliques. Such a relationship could possibly explain the imperfect match between the effects of isotropic experience and oblique dominant experience if the prior is made up of two parts. Forcing a relationship between orthogonal orientations into the model may also help elucidate how and where (cortically) the orthogonal facilitation is happening. All of these ideas are speculative and would need to be tested.

Based on the perceptual changes demonstrated above, it can be concluded that the visual system is continually updating the likelihood distributions across oriented structure to more accurately reflect the statistics of the current visual environment. As indicated by the model, it appears that the visual system is employing a Bayesian approach to representing orientation information in the environment, updating the likelihood distribution in response to changes in the predictability of environmental statistics. The representation of the likelihood distribution in Bayesian perceptual models necessarily relates to the variability in the sensory measurement as it is the information available to the model. The likelihood must change, then, in response to adaptation as the neural encoding of the information (sensory noise) is known to increase the gain (SNR) of signals near the adapting values (Brenner et al., 2000; Clifford et al., 2007; Dragoi, 
Sharma, \& Sur, 2000; Ohzawa, Scalr, \& Freeman, 1985). As previously argued, the prior is less likely to change as it is presumed to represent past experience with the probability of encountering certain stimuli in the external world (Stocker \& Simoncelli, 2006b). However, assuming that the more efficient way of representing the global environmental statistics is to whiten the signal, the prior may also change to counteract the effects of increasing the signal to noise ratio near the adapting values. These types of changes would not be necessarily required unless the environment was changing on a global level (as in Experiment 4).

As previously pointed out, the advantage of using a Bayesian model of perception is that the likelihood and prior can be modeled from data obtained under circumstances which differ from those in this experiment (Girshick, Landy, \& Simoncelli, 2011). As shown here, in circumstances where the global distribution of orientations changes, one would expect observers' priors to change as well which, as discussed below, may correlate to change in spontaneous activity. Although some have posited that the prior may take significant time to develop as it is based on the environment (Girshick, Landy, \& Simoncelli, 2011), others have shown that priors can indeed change during the course of adaptation (Kording \& Wolpert, 2004; Tassinari, Hudson, \& Landy, 2006; Wei, Ortega, \& Stocker, 2015). In a related line, Chopin and Mamassian (2012) have recently discovered that there may be more of a hierarchical relationship between adapting to recent stimuli and more remote experience. In their study, the authors showed that the percept that results from adaption can be predicted from assuming that it will be that percept which helps the most recent experience match that of the more remote past 
(Chopin \& Mamassian, 2012). In other words, observers' perception is biased not only by more long-term experience with their environment but also by the recent environmental exposure.

In fact, more recently a Bayesian model of visual adaptation has been expanded to encompass changes in both the likelihood and prior. Specifically, the history of input to the visual system should be used to predict future input under a Bayesian framework (Wei \& Stocker, 2012; Wei, Ortega, \& Stocker, 2015). In order to predict the future well, the priors must be adjusted when the input to the system changes (Chopin \& Mamassian, 2012). While moment-to-moment adjustments in visual input can enact changes in the likelihood distribution that are sufficient to explain perceptual changes, longer-term changes should also affect the prior. As changes in likelihoods tend to bias perception away from the adapting value, such a model predicts decreases in threshold at orthogonal orientations as well (Wei \& stocker, 2012; Wei, Ortega, \& Stocker, 2015). As an observer gains more experience with the altered environment, the existing prior should become a better match to the new environment than the old as new input is integrated into existing experience (Chopin \& Mamassian, 2012). Modeling the change in Experiment 1 across adaptation duration should also capture how an observer's prior might change gradually from one that is biased to match the natural environment to one that is a closer match to the experienced altered environment.

As discussed in the Introduction, spontaneous neural activity could represent the prior under a Bayesian representation of neural population coding (Fiser et al., 2010). Spontaneous activity has been shown to correlate with stimulus evoked activity and this 
correlation increases with developmental age, but is eliminated when visual input is disrupted (Berkes et al., 2011; Fiser et al., 2013; Kenet et al., 2003; Tsodyks et al., 1999). It would be interesting to determine if spontaneous activity changes after experience in an altered environment. If this is the case, then the change in prior activated by adapting to changes in environmental statistics should also produce a change in spontaneous activity. Presumably, experience in environments such as those tested here would change the population code during adaptation and spontaneous activity after adaptation would more closely match the adapted distribution than the typical distribution. Assuming spontaneous activity has changed, it would then take longer to return to a natural prior the more the activity deviated. That is, larger changes in the environmental distribution should produce longer lasting effects of adaptation as it will take longer for the change in activity to return to baseline given post-adaptation experience in an anisotropic environment. As Figure 20 shows, the larger physical distribution deviation did indeed have longer lasting effects.

One interesting future investigation would be to determine how the perceptual biases -- presumably related to the environmental input one experiences -- develop. To date, no one has examined the existence of a horizontal effect in children or infants. Although this would be difficult to test, it would be necessary to determine when the perceptual biases that are related to environmental experience arise. There is some suggestion that the efficient processing of some visual statistics may not develop until late childhood indicating a starting point for future investigations (Ellemberg, Hansen, \& Johnson, 2012; Ellemberg et al., 2012; Pei, Baldassi, \& Norcia, 2012). If, indeed, the 
perceptual adaptability uncovered in these experiments does not develop until later as well, it would also be of interest to determine the course of the development itself. Moreover, there is a captivating question as to how long this adaptability remains. Is there a critical period for environmental adjustment of the prior as there is for visual development? It would also be interesting to test the effects of adapting to altered environments in older populations. If there is indeed a limit to the malleability, as possibly indicated in Experiment 4, is this limit more plastic in children or adolescents? Clearly, the development track of this adaptability is fascinating and should be the subject of future study.

Theoretically, perceptual learning could implement the same kind of adaptive adjustment of the prior seen in these adaptation experiments. However, there are two main differences between traditional perceptual learning studies and the results reported here: perceptual learning is stimulus specific (Sowden, Davies, \& Roling, 2000; Mayer, 1983), but not relative (Mayer, 1983; Song et al., 2010; Vogels, \& Orban, 1985; Westheimer \& Lavian, 2013), and suggests memory for learning (Hofman, Van Riswick, \& Van Opstal 1998). Much of the perceptual learning literature has shown that while training can improve performance, the improvements are very specific to the trained stimuli (Sowden, Davies, \& Roling, 2000; Mayer, 1983). Moreover, while training improves performance for trained stimuli, it does not cause a relative decrement in performance at untrained stimuli (Mayer, 1983; Song et al., 2010; Vogels, \& Orban, 1985; Westheimer \& Lavian, 2013). Both of these findings are in opposition with those reported above; experience in an altered environment effected perception of novel noise 
stimuli and was stimulus-relative such that improvements at specific orientations necessarily imposed decrements at other orientations. Furthermore, whereas adaptation to altered environmental statistics caused a shift in observers' priors that was maintained in some cases for the entire post-testing period, adaptation studies in other modalities have shown immediate return to baseline levels (Hofman, Van Riswick, \& Van Opstal 1998). For example, after participants had been trained to localize sounds with a modified outer ear (pinnae), they were still able to localize sounds immediately after the removal of the artificial pinnae (Hofman, Van Riswick, \& Van Opstal 1998). This finding seems to indicate that perceptual learning involves memory for the learned stimuli while the results reported above do not indicate a role of memory; after adaptation, subjects required visual input in the typical environment to return to baseline perceptual biases (Figure 24). For these reasons it seems likely that, although perceptual learning may be enacted under a Bayesian framework, it likely employs different mechanisms of action.

Some have supposed that the relationships between natural scene statistics and visual processing evolved across millennia in order to make the visual system an efficient information-transmitting system (Bex, Solomon, \& Dakin, 2009; Brenner, et al., 2000; Essock et al., 2003; Essock, Haun, \& Kim, 2009; Hansen \& Essock, 2004; 2006; Haun \& Essock, 2010; Webster \& Miyahara, 1997). However, given that the natural world is continually experienced, perception should be optimized to encode incoming statistical information, adjusting dynamically to the input (Gutnisky \& Dragoi, 2008; Wainwright, 1999). This work shows that perceptual encoding can indeed adjust to the regularities in 
incoming signals such that the perceptual result is optimized to the most current input. Though there may be a limit to the system's plasticity (i.e. the oblique condition), the experiments detailed above have shown that the biases of visual perception that relate to typical scene statistics can be altered. This work indicates that observers have an accurate representation of the regularities present in their recent environment that informs their perception of features in context. While the 'typically' experienced distribution leads to the anisotropic bias of the horizontal effect, altering the experienced orientation distribution causes predictable changes in orientation perception: a globally isotropic orientation spectrum causes subjects to become more isotropic in orientation perception as well as lessen their pre-adaptation orientation biases. These results show that perceptual encoding can indeed adjust to the regularities in incoming signals such that the perceptual result is optimized to the most current input. Moreover, these changes appear to go beyond short-term changes in likelihoods. These results implicate changes in prior distributions in perceptual adaptation to atypical orientation distributions. This suggests that rather than being adapted to distributional information that has been in our environments for millennia, perceptual biases reflect an internal model in which likelihood and priors adapt dynamically to recent experience.

The prevailing notion in the recent literature has been that visual processing is matched to natural scenes statistics because of evolutionary adaptations, but this work suggests otherwise. As discussed in detail in the Introduction, the processing of different orientations by our visual system is biased and has an inverse relationship to the orientation content present in natural scenes. The original work by Essock and 
colleagues $(2003,2004,2005,2006,2009,2010)$ hypothesized that this relationship may have developed across the millennia due to the constant bombardment of the sensory system with a biased orientation distribution in the environment. Until this study, no one had examined whether or not changes in the statistics of one's visual environment would change the horizontal effect of orientation processing. Logically, if the environmental statistics of one's current visual environment are in fact determining perception, and this perception is malleable, then horizontal effect should be alterable given different experience with a world in which the orientation distribution is 'unnatural.' These adaptation paradigms have shown just that; one can alter the horizontal effect simply by changing an observer's environment. This project had indicated that the brain is a very plastic, efficient, and probabilistic encoder. Moreover, it shows that the horizontal effect is an effect of adaptation to environmental statistics, most likely due to Bayesian frameworks in the visual system, which can change how the brain is encoding information 'on the fly.' While all of these experiments tested visual orientation perception, there is no reason to assume that the adaptive responses seen here in response to changing orientation statistics could not also occur with other types of visual input as well as in other modalities. 


\section{REFERENCES}

Abbonizo, G., Langley, K., \& Clifford, C.W.G. (2002). Contrast adaptation may enhance contrast discrimination. Spatial Vision. 16(1), 45-58.

Appelle, S. (1972). Perception and discrimination as a function of stimulus orientation: Oblique effect in man and animals. Psychological Bulletin, 78(4), 266-278.

Aspell JE, Wattam-Bell J, Atkinson J, \& Braddick OJ. (2010). Differential human brain activation by vertical and horizontal global visual textures. Experimental Brain Research. 202(3):669-79.

Atick, J. J., \& Redlich, A. N. (1990). Towards a theory of early visual processing. Neural Computation, 2(3), 308-320.

Atick, J. J., \& Redlich, A. N. (1992). What does the retina know about natural scenes?. Neural computation, 4(2), 196-210.

Atkinson, J., Hood, B., Wattam-Bell, J., Anker, S., \& Tricklebank, J. (1988). Development of orientation discrimination in infancy. Perception, 17, 587-595. 
Attneave, F. (1954). Some informational aspects of visual perception.Psychological review, 61(3), 183.

Attneave, F. \&Olson, R.K. (1967). Discriminability of stimuli varying in physical and retinal orientation. Journal of Experimental Psychology, 74(2), 149-157.

Baddeley, R. J., \& Hancock, P. J. B. (1991). A statistical-analysis of natural images matches psychophysically derived orientation tuning curves. Proceedings of the Royal Society of London Series B-Biological Sciences, 246(1317), 219-223.

Bao, M. \& Engel, S.A. (2012). Distinct mechanism for long-term contrast adaptation. Proceedings fo the National Academy of Science, 109(15), 5898-5903.

Bao, M., Fast, E., Mesik, J., \& Engel, S. (2013). Distinct mechanisms control contrast adaptation over different timescales. Journal of vision, 13(10), 14.

Barlow, H. B. (1961). Possible principles underlying the transformation of sensory messages. Sensory communication, 217-234.

Barlow, H. (1989). Unsupervised learning. Neural Computation, 1(3), 295-311.

Barlow, H. (2001). Redundancy reduction revisited. Network: Computational Neural Systems 12, 241-253. 
Beck, J.M., Ma, W.J., Kiani, R., Hanks, T., Churchland, A.K., Roltman, J., Shadlen, M.N., Latham, P.E., \& Pouget, A. (2008). Probabilistic population codes for Bayesian decision making. Neuron, 60, 1142-1152.

Berkes, P., Orban, G., Lengyel, M., \& Fiser, J. (2011). Spontaneous cortical activity reveals hallmarks of an optimal internal model of the environment. Science, 331, 8-3-87.

Bex, P.J., Solomon, S.G. \& -Dakin, S.C. (2009). Contrast sensitivity in natural scenes depends on edge as well as spatial frequency structure. Journal of Vision 9, 1, 119.

Blakemore, C. \& Campbell, F.W. (1969). On the existence of neurons in the human visual system selectively sensitive to the orientation and size of retinal images. Journal of Physiology, 203, 237-260.

Brenner, N., Bialek, W., \& de Ruyter van Steveninck, R. (2000). Adaptive rescaling maximizes information transmission. Neuron, 26, 695-702.

Butler, A. J., James, T. W., \& James, K. H. (2011). Enhanced multisensory integration and motor reactivation after active motor learning of audiovisual associations. Journal of cognitive neuroscience, 23(11), 3515-3528.

Campbell, F.W. \& Kulikoski, J.J. (1966). Orientational selectivity of the human visual system. Journal of Physiology, 187, 437-445. 
Chapman, B. \& Bonhoeffer, T. (1994). Chronic optical imaging of the development of orientation domains in ferret area 17. Society of Neuroscience Abstract, 20, 214.

Chapman B, Stryker MP, \& Bonhoeffer T.(1998). Overrepresentation of horizontal and vertical orientation preferences in developing ferret area 17. Proceedings of the National Academy of Science. 95(5):2609-14.

Chen, C. C., \& Tyler, C. W. (2002). Lateral modulation of contrast discrimination: Flanker orientation effects. Journal of Vision, 2(6), 8.

Chopin, A. \& Mamassian, P. (2012). Predictive properties of visual adaptation. Current Biology, 22(7), 622-626.

Clifford,C., Mannion, D., \& McDonald, J. (2011). Orientation anisotropies in human visual cortex complement those in natural scenes. Perception, 40, 107-107.

Clifford, C.W.G., Webster, M.A., Stanley, G.B., Stocker, A.A., Kohn, A., Sharpee, T.O., \& Schwartz, O. (2007). Visual Adaptation: Neural, psychological and computational aspects. Vision Research, 47, 3125-3131.

Coppola, D. M., Purves, H. R., McCoy, A. N., \& Purves, D. (1998). The distribution of oriented contours in the real word. Proceedings of the National Academy of Sciences of the United States of America, 95(7), 4002-4006.

Corwin, T. R., Moskowitz-Cook, A., \& Green, M. A. (1977). The oblique effect in a vernier acuity situation. Perception \& Psychophysics, 21(5), 445-449. 
De Valois RL, Yund EW, \& Hepler N.(1982). The orientation and direction selectivity of cells in macaque visual cortex. Vision Research. 22(5):531-44.

Dragoi, V., Sharma, J., \& Sur, M. (2000). Adaptation-induced plasticity of orientation tuning in adult visual cortex. Neuron, 28(1), 287-298.

Dragoi, V. Turcu, C. M. Sur, M. (2001). Stability of cortical responses and the statistics of natural scenes. Neuron, 32(6), 1181-1192.

Ellemberg, D., Hansen, B.C., \& Johnson, A. (2012). The developing visual system is not optimally sensitive to the spatial statistics of natural images. Vision Research, 67, $1-7$.

Ellemberg, D., St Jacques, S., St Louis Deschenes, M., Baillargeon Blais, A., \& Labonte Lemoyne, E. (2012). Developmental processes underlying orientation discrimination during childhood. ECVP.

Emsley, H.H. (1925). Irregular astigmatisms of the eye: Effect of correcting lenses. Transactions of the Optical Society, 27, 235-237.

Essock, E.A. (1980). The oblique effect of stimulus identification considered with respect to two classes of oblique effects. Perception, 9, 37-46.

Essock, E.A. (1982). Anisotropies of perceived contrast and detection speed. Vision Research, 22, 1185-1191. 
Essock, E. A., DeFord, J. K., Hansen, B. C., \& Sinai, M. J. (2003). Oblique stimuli are seen best (not worst!) in naturalistic broadband stimuli: A horizontal effect. Vision Research, 43, 1329-1335.

Essock, E. A., Krebs, W. K., \& Prather, J. R. (1997). Superior sensitivity for tactile stimuli oriented proximally-distally on the finger: Implications for mixed class 1 and class 2 anisotropies. Journal of Experimental Psychology: Human Perception and Performance, 23(2), 515.

Essock, E. A., Haun, A. M., \& Kim, Y. (2009). An anisotropy of orientation-tuned suppression that matches the anisotropy of typical natural scenes. Journal of Vision, 9(1).

Essock, E. A. \& Siqueland, E. R. (1981). Discrimination of orientation by human infants. Perception, 10(3), 245-253.

Feldman, J. (2013). Tuning your priors to the world. Topics in cognitive science, 5(1), 1334.

Field, D. J. (1987). Relations between the statistics of natural images and the response properties of cortical-cells. Journal of the Optical Society of America a-Optics Image Science and Vision, 4(12), 2379-2394.

Field, D. J., \& Brady, N. (1997). Visual sensitivity, blur and the sources of variability in the amplitude spectra of natural scenes. Vision Research, 37, 3367-3383. 
Fiser, J. \& Aslin, R.N. (2001). Unsupervised statistical learning of higher-order spatial structures from visual scenes. Psychological Science, 12(6), 499-505.

Fiser, J. \& Aslin, R.N. (2005). Encoding multielement scenes: Statistical leanring of visual feature hierarchies. Journal of Experimental Psychology: General, 134(4), 521-537.

Fiser, J., Berkes, P., Orban, G., \& Lengyel, M. (2011). Statistically optimal perception and learning: From behavior to neural representations. Trends in Cognitive Sciences, 14(3), 119-130.

Fiser, J., Savin, C., Berkes, P., Chiu, C., \& Lengyel, M. (2013). Experience-based development of internal probablisitic representations in the primary visual cortex. Vision Sciences Society Annual Meeting, Naples, FL, May 2013.

Foley, J.M. (1994). Human luminance pattern-vision mechanisms: Masking experiments require a new model. Journal of the Optical Society of America, 11, 1710-1719.

Fredembach, B., de Boisferon, A. H., \& Gentaz, E. (2009). Learning of arbitrary association between visual and auditory novel stimuli in adults: the "bond effect" of haptic exploration. PloS one, 4(3), e4844.

Freeman, J., Brouwer, G. J., Heeger, D. J., \& Merriam, E. P. (2011). Orientation decoding depends on maps, not columns. The Journal of Neuroscience, 31(13), 4792-4804. 
Freeman, J., Heeger, D.J., \& Merriam, E.P. (2013). Coarse-scale biases for spirals and orientation in human visual cortex. The Journal of Neuroscience, 35(50), 1969519703.

Furmanski CS, \& Engel SA.(2000). An oblique effect in human primary visual cortex. Nature Neuroscience. 3(6):535-6.

Furmanski, C. S., Schluppeck, D., \& Engel, S. A. (2004). Learning strengthens the response of primary visual cortex to simple patterns. Current Biology, 14(7), 573578.

Gilinsky, A.S. (1968). Orientation-specific effects of patterns of adapting light on visual acuity. Journal of the Optical Society of America, 58(1), 13-18.

Girshick, A. R., Landy, M. S., \& Simoncelli, E. P. (2011). Cardinal rules: visual orientation perception reflects knowledge of environmental statistics. Nature Neuroscience,14(7), 926-156.

Grabaska-Barwinska, A., Distler, C., Hoffman, K., \& Jancke, D. (2009). Contrast independence of cardinal preference: stable oblique effect in orientation maps of ferret visual cortex. European Journal of Neuroscience 29, 1258-1270.

Graham, D. J., Chandler, D. M., \& Field, D. J. (2006). Can the theory of "whitening" explain the center-surround properties of retinal ganglion cell receptive fields?. Vision research, 46(18), 2901-2913. 
Greenlee, M.W. \& Heitger, F. (1988). The functional role of contrast adaptation. Vision Research 28(7) 791-797.

Gutnisky, D. A., \& Dragoi, V. (2008). Adaptive coding of visual information in neural populations. Nature, 452(7184), 220-224.

Haak, K.V., Fast, E., Bao, M., Lee, M., \& Engel, S.A. (2014). Four days of visual contrast deprivation reveals limits of neuronal adaptation. Current Biology, 24(21). 2575-2579.

Hamblin, J. R. \& Winser, T.H. (1927). On resolution of gratings by the astigmatic eye. Transactions of the Optical Society, 29, 28-42.

Hansen, B.C., Essock, E.A., Zheng, Y., \& DeFord, J.K. (2003). Perceptual anisotropies in visual processing and their relation to natural image statistics. Network: Computation in Neural Systems, 14, 501-526.

Hansen, B. C., \& Essock, E. A. (2004). A horizontal bias in human visual processing of orientation and its correspondence to the structural components of natural scenes. Journal of Vision, 4(12), 1044-1060.

Hansen, B. C. \& Essock, E. A. (2006). Anisotropic local contrast normalization: The role of stimulus orientation and spatial frequency bandwidths in the oblique and horizontal effect perceptual anisotropies. Vision Research, 46(26), 4398-4415.

Hansen, B.C. \& Hess, R.F. (2006). Discrimination of amplitude spectrum slope in the fovea and parafovea and the local amplitude distributions of natural scene imagery. Journal of Vision 6, 696-711. 
Hansen, B. C., \& Hess, R. F. (2007). Structural sparseness and spatial phase alignment in natural scenes. JOSA A, 24(7), 1873-1885.

Hansen, B.C., Richard, B., Andres, K.A., Johnson, A.P., \& Essock, E.A., (under review). A cortical locus for anisotropic overlay suppression of stimuli presented at fixation.

Haun, A. M. \& Essock, E. A. (2010). Contrast Sensitivity for orientated patterns in 1/f noise: Contrast response and the horizontal effect. Journal of Vision, 10(10).

Haun, A. M., \& Peli, E. (2013). Perceived contrast in complex images. Journal of vision, 13(13), 3 .

Heinrich, S. P., Aertsen, A., \& Bach, M. (2008). Oblique effects beyond low-level visual processing. Vision research, 48(6), 809-818.

Higgins, G.C. \& Stultz, K. (1950). Variation of visual acuity with various test-object orientations and viewing conditions. Journal of the Optical Society of America, $40,135-137$.

Hofman, P. M., Van Riswick, J. G., \& Van Opstal, A. J. (1998). Relearning sound localization with new ears. Nature neuroscience, 1(5), 417-421.

Huang L, Shou T, Chen X, Yu H, Sun C, \& Liang Z.(2006). Slab-like functional architecture of higher order cortical area 21a showing oblique effect of orientation preference in the cat. Neuroimage. 32(3):1365-74. 
Hubel, D. H., \& Wiesel, T. N. (1959). Receptive fields of single neurones in the cat's striate cortex. The Journal of physiology, 148(3), 574.

Hubel, D. H., \& Wiesel, T. N. (1962). Receptive fields, binocular interaction and functional architecture in the cat's visual cortex. The Journal of physiology,160(1), 106.

James, K. H., Humphrey, G. K., \& Goodale, M. A. (2001). Manipulating and recognizing virtual objects: where the action is. Canadian Journal of Experimental Psychology/Revue canadienne de psychologie expérimentale,55(2), 111

James, K. H., Humphrey, G. K., Vilis, T., Corrie, B., Baddour, R., \& Goodale, M. A. (2002). "Active" and "passive" learning of three-dimensional object structure within an immersive virtual reality environment. Behavior Research Methods, Instruments, \& Computers, 34(3), 383-390.

Jehee, J. F., Ling, S., Swisher, J. D., van Bergen, R. S., \& Tong, F. (2012). Perceptual learning selectively refines orientation representations in early visual cortex. The Journal of Neuroscience, 32(47), 16747-16753.

Johnson, A.P., Richard, B., Hansen, B.C., \& Ellemberg, D. (2011). The magnitude of center-surround facilitation in the discrimination of amplitude spectrum is dependent on the amplitude of the surround. Journal of Vision, 11 (7), 1-10.

Kamitani, Y., \& Tong, F. (2005). Decoding the visual and subjective contents of the human brain. Nature neuroscience, 8(5), 679-685. 
Keehner, M., Hegarty, M., Cohen, C., Khooshabeh, P., \& Montello, D. R. (2008). Spatial reasoning with external visualizations: What matters is what you see, not whether you interact. Cognitive Science, 32(7), 1099-1132.

Keil, M. S., \& Cristobal, G. (2000). Separating the chaff from the wheat: possible origins of the oblique effect. Journal of the Optical Society of America a-Optics Image Science and Vision, 17(4), 697-710.

Kelly, J. W., Donaldson, L. S., Sjolund, L. A., \& Freiberg, J. B. (2013). More than just perception-action recalibration: Walking through a virtual environment causes rescaling of perceived space. Attention, Perception, \& Psychophysics,75(7), $1473-1485$.

Kenet, T., Bibitchkov, D., Tsodyks, M., Grinvald, A., \& Areli, A. (2003). Spontaneously emerging cortical representations of visual attributes. Nature 425, 954-956.

Kennedy, H., Martin, K. A. C., Orban, G. A., \& Whitteridge, D. (1985). Receptive field properties of neurones in visual area 1 and visual area 2 in the baboon. Neuroscience, 14(2), 405-415.

Kim, Y. J., Haun, A. M., \& Essock, E. A. (2010). The horizontal effect in suppression: Anisotropic overlay and surround suppression at high and low speeds. Vision research, 50(9), 838-849.

Knill, D. C., Field, D., \& Kersten, D. (1990). Human discrimination of fractal images. Journal of the Optical Society of America A, Optics and Image Science, 7, 11131123 
Koelewijn L, Dumont JR, Muthukumaraswamy SD, Rich AN, \& Singh KD.(2011). Induced and evoked neural correlates of orientation selectivity in human visual cortex. Neuroimage. 54(4):2983-93.

Kohn, A. (2007). Visual Adaptation: Physiology, mechanisms, and function benefits. Journal of Neurophysiology, 97, 3155-3164.

Körding, K. P., \& Wolpert, D. M. (2004). Bayesian integration in sensorimotor learning. Nature, 427(6971), 244-247.

Kwon, M., Legge, G. E., Fang, F., Cheong, A. M., \& He, S. (2009). Adaptive changes in visual cortex following prolonged contrast reduction. Journal of vision, 9(2), 20.

Ledgeway, T., Hess, R. F., \& Geisler, W. S. (2005). Grouping local orientation and direction signals to extract spatial contours: Empirical tests of "association field" models of contour integration. Vision research, 45(19), 2511-2522.

Leehey, S. C., Moskowitz-Cook, A., Brill, S., \& Held, R. (1975). Orientational Anisotropy in infant vision. Science, 190(4217) 900-902.

Leibowitz, H. (1953). Some observations and theory on the variation of visual acuity with the orientation of the test object. Journal of the Optical Society of America, 43, 902-905.

Leopold, D. A., Rhodes, G., Müller, K. M., \& Jeffery, L. (2005). The dynamics of visual adaptation to faces. Proceedings of the Royal Society B: Biological Sciences, 272(1566), 897-904. 
Leventhal, A.G., \& Hirsch, H. V. B. (1977). Effects of early experience upon orientation sensitivity and binocularity of neurons in visual cortex of cats. Proceedings of the National Academy of Sciences, 74, 1272-1276.

Li B, Peterson MR, \& Freeman RD.(2003). Oblique effect: a neural basis in the visual cortex. Journal of Neurophysiology. 90(1):204-17.

Liang, Z., Shen, W., \& shou, T. (2007). Enhancement of oblique effect in the cat's primary visual cortex via orientation preference shifting induced by excitatory feedback from higher-order cortical area 21A. Neuroscience. 145, 377-383.

Maffei, L., \& Campbell, F. W. (1970). Neurophysiological localization of the vertical and horizontal visual coordinates in man. Science, 167(3917), 386-387.

Mamassian, P., Landy, M., \& Maloney, L. (2002). Bayesian modeling of visual perception. In Rao, R.P.N., Olshausen, B.A., \& Lewicki, M.S. (Eds.) Probabilistic Models of the Brain: Percetion and Neural Function. Cambridge, MA: MIT Press, $13-36$.

Mannion DJ, McDonald JS, \& Clifford CW. (2010a). Orientation anisotropies in human visual cortex. Journal of Neurophysiology. 103(6):3465-71.

Mannion, D. J., McDonald, J. S., \& Clifford, C. W. (2010b). The influence of global form on local orientation anisotropies in human visual cortex.Neuroimage, 52(2), $600-605$. 
Mansfield, R.J.W. (1974). Neural basis of orientation perception in primate vision, Science 186 133-135.

Mansfield, R.J.W. \& Ronner, S.F. (1978). Orientation anisotropy in monkey visual cortex. Brain Research, 149(1), 229-234.

Marx, S., \& Einhäuser, W. (2015). Reward modulates perception in binocular rivalry. Journal of vision, 15(1), 11.

Mayer, M. J. (1983). Practice improves adults' sensitivity to diagonals. Vision research, 23(5), 547-550.

McDonald, J.S., Mannion, D.J., \& Clifford, C.W. (2012). Gain control in the response of human visual cortex to plaids. Journal of Neurophysiology. 107. 2570-2580.

Mesik, J., Patke, A., \& Engel, S. A. (2015, May). Repeatedly adapting to orientation ensembles does not change contrast adaptation dynamics. Post presented at the annual meeting of the Vision Sciences Society, St. Pete's Beach, FL.

Morgan, M. J., Ross, J., \& Hayes, A. (1991). The relative importance of local phase and local amplitude in patchwise image reconstruction. Biological Cybernetics, 65(2), 113-119.

Muller J.R., Metha, A.B., Krauskopf, J., \& Lennie, P. (1999). Rapid adaptation in visual cortex to the structure of images. Science. 285, 1405-1408.

Nagai, M., Suzuki, W., \& Wang, G. (2007). Functional map of orientation tuning width and specificity. NeuroReport, 18(13), 1357-1360. 
Nankoo, J., Madan, C.R., Spetch, M.L., \& Wylie, D.R. (2012). Perception of dynamic Glass patterns. Vision Research, 72, 55-62.

Nasr, S., \& Tootell, R. B. (2012). A cardinal orientation bias in scene-selective visual cortex. The Journal of Neuroscience, 32(43), 14921-14926.

Ohzawa, I., Sclar, G., \& Freeman, R.D. (1985). Contrast gain control in the cat's visual system. Journal of Neurophysiology, 54(3), 651-667.

Oliva, A., \& Torralba, A. (2001). Modeling the shape of the scene: A holistic representation of the spatial envelope. International journal of computer vision,42(3), 145-175.

Oppenheim, A. V., \& Lim, J. S. (1981). The importance of phase in signals.Proceedings of the IEEE, 69(5), 529-541.Pei, F., Baldassi, S., \& Norcia, A. (2012). Late Development of contrast normalization mechanisms. ECVP, Alghero, Italy.

Orban, G. A., \& Kennedy, H. (1981). The influence of eccentricity on receptive field types and orientation selectivity in areas 17 and 18 of the cat. Brain Research, 208, 203-208.

Pa'rraga, C. A., Troscianko, T., \& Tolhurst, D. J. (2000). The human visual system is optimized for processing the spatial information in natural visual images. Current Biology, 10, 35-38.

Pei, F., Baldassi, S., \& Norcia, A. (2012). Late development of contrast normalization mechanisms. Perception, 41, 51-51. 
Piotrowski, L. N., \& Campbell, F. W. (1982). A demonstration of the visual importance and flexibility of spatial-frequency amplitude and phase. Perception.

Powers, A.R., Hillock, A.R., Wallace, M.T. (2009). Perceptual training narrows the temporal window of multisensory binding. The Journal of Neuroscience, 29(39), $12265-12274$.

Pouget, A., Dayan, P., \& Zemel, R. (2003). Inference and computation with population codes. Annual Review of Neuroscience 26, 381-410.

Prather, J. R., Krebs, W. K., Sinai, M. J., McCarley, J. S., \& Essock, E. A. (1995). Oblique orientations are confused in visual search. Investigative Ophthalmology and Visual Science 36(Suppl. 4), 900.

Proverbio AM, Esposito P, \& Zani A.(2002). Early involvement of the temporal area in attentional selection of grating orientation: an ERP study. Brain Research. 13(1):139-51.

Quinn, P.C. \& Bomba, P.C. (1986). Evidence for a general category of oblique orientations in four-month-old infants. Journal of Experimental Child Psychology, $42,345-354$.

Quinn, P.C., Siqueland, E.R., \& Bomba, P.C. (1985). Delayed rec, ognition memory for orientation by human infants. Journal of Experimental Child Psychology, 40, 293303.

Regan, D. \& Beverly, K.L. (1985). Postadaptation orientation discrimination. Journal of the Optical Society of America, 2(2), 147-155. 
Sawides, L., de Gracia, P., Dorronsoro, C., Webster, M.A., \& Marcos, S. (2011). Vision is adapted to the natural level of blur present in the retinal image. PLoS one, $6(11)$

Schwartz, O., \& Simoncelli, E. P. (2001). Natural signal statistics and sensory gain control. Nature neuroscience, 4(8), 819-825.

Schweinhart, A. M. (2014). Effects of Recent Exposure to Atypical Environmental Statistics on Orientation Perception: Analyzing the Plasticity of the Horizontal Effect. Unpublished manuscript, Department of Psychological and Brain Sciences, University of Louisville, Louisville, KY United States.

Schweinhart, A. M., \& Essock, E. A. (2013). Structural content in paintings: Artists overregularize oriented content of paintings relative to the typical natural scene bias. Perception, 42, 1311-1332.

Serences, J. T., Saproo, S., Scolari, M., Ho, T., \& Muftuler, L. T. (2009). Estimating the influence of attention on population codes in human visual cortex using voxelbased tuning functions. Neuroimage, 44(1), 223-231.

Shapley, R., Caelli, T., Grossberg, S., Morgan, M., \& Rentschler, I. (1990). Computational theories of visual perception. Visual perception: The neurophysiological foundations, 417-448.

Shen, W., Liang, Z., \& Shou, T. (2008). Weakened feedback abolishes neural oblique effect evoked by pseudo-natural visual stimuli in area 17 of the cat.Neuroscience letters, 437(1), 65-70. 
Shen, G., Tao, X., Zhang, B., Smith, E. L., \& Chino, Y. M. (2014). Oblique effect in visual area 2 of macaque monkeys. Journal of vision, 14(2), 3.

Shlaer, S. (1937). The relation between visual acuity and illumination. Journal of the General Phsyiology, 21, 165-188.

Simoncelli, E. P., \& Olshausen, B. A. (2001). Natural image statistics and neural representation. Annual review of neuroscience, 24(1), 1193-1216.

Sokol, S., Moskowitz, A. \& Hansen, V. (1987). Electrophysiological evidence for the oblique effect in human infants. Investigative ophthalmology and vision, 28(4), 731-735.

Song, Y., Sun, L., Wang, Y., Zhang, X., Kang, J., Ma, X., Yang, B., Guan, Y., Ding, Y. (2010). The effect of shirt-term training on cardinal and oblique orientation discrimination: An ERP study. International Journal of Psychophysiology, 75, 241-248.

Stocker, A., \& Simoncelli, E. P. (2006a). Sensory adaptation within a Bayesian framework for perception. Advances in neural information processing systems, 18 , 1289.

Stocker, A. \& Smioncelli, E.P. (2006b). Noise Characteristics and prior expectations in human visual speed perception. Nature Neuroscience, 9(4), 578-584.

Sun, P., Gardner, J. L., Costagli, M., Ueno, K., Waggoner, R. A., Tanaka, K., \& Cheng, K. (2013). Demonstration of tuning to stimulus orientation in the human visual 
cortex: a high-resolution fMRI study with a novel continuous and periodic stimulation paradigm. Cerebral Cortex, 23(7), 1618-1629.

Sun, M., Huang, J., Wang, F., An, A., Tian, F., Liu, H., Niu, H., \& Song, Y. (2013). Quantitative comparison of the hemodynamic activation elicited by cardinal and oblique gratings with functional near-infared spectroscopy. NeuroReport, 24(7), 354-358.

Swisher, J. D., Gatenby, J. C., Gore, J. C., Wolfe, B. A., Moon, C. H., Kim, S. G., \& Tong, F. (2010). Multiscale pattern analysis of orientation-selective activity in the primary visual cortex. The Journal of Neuroscience, 30(1), 325-330.

Switkes, E., Mayer, M. J., \& Sloan, J. A. (1978). Spatial frequency analysis of the visual environment: Anisotropy and the carpentered environment hypothesis. Vision research, 18(10), 1393-1399.

Tadmor, Y., \& Tolhurst, D. J. (1994). Discrimination of changes in the second-order statistics of natural and synthetic images. Vision Research, 34, 541-554.

Tassinari, H., Hudson, T. E., \& Landy, M. S. (2006). Combining priors and noisy visual cues in a rapid pointing task. The Journal of neuroscience,26(40), 10154-10163.

Tolhurst, D. J., Tadmor, Y., \& Chao, T. (1992). Amplitude spectra of natural images. Ophthalmic and Physiological Optics, 12, 229-232.

Tsodyks, M.,Kenet, T., Grinvald, A., \& Arieli, A. (1999). Linking spontaneous activity of single cortical neurons and the underlying functional architecture. Science 286, 1943-1946. 
Turk-Browne, N.B., Scholl, B.J., Chun, M.M., \& Johnson, M.K. (2009). Neural evidence of statistical learning: efficient detection of visual regularities without awareness. Journal of Cognitive Neuroscience, 21(10), 1934-1945.

van der Schaaf, V. A., \& van Hateren, J. V. (1996). Modelling the power spectra of natural images: statistics and information. Vision research, 36(17), 2759-2770.

Vogels, R., \& Orban, G. A. (1985). The effect of practice on the oblique effect in line orientation judgments. Vision research, 25(11), 1679-1687.

Wainwright, M. J. (1999). Visual adaptation as optimal information transmission. Vision research, 39(23), 3960-3974.

Wang, G., Ding, S., \& Yunokuchi, K. (2003). Difference in the representation of cardinal and oblique contours in cat visual cortex. Neuroscience letters, 338(1), 77-81.

Webster, M.A. (2011). Adaptation and visual coding. Journal of Vision, 11(5), 1-23.

Webster, M.A., Georgeson, M.A., \& Webster, S.M. (2002). Neural adjustments to image blur. Nature Neuroscience, 5(9), 839-840.

Webster, M. A., \& Miyahara, E. (1997). Contrast adaptation and the spatial structure of natural images. Journal of the Optical Society of America, 14(9), 2355-2366.

Wei, X. X., \& Stocker, A. (2012). Efficient coding provides a direct link between prior and likelihood in perceptual Bayesian inference. In Advances in neural information processing systems (pp. 1313-1321). 
Wei, X.X., Ortega, P. \& Stocker, A. (2015, May). Perceptual Adaptation: Getting ready for the future. Poster presented at the Vision Sciences Society Annual Meeting. St. Petersburg, FL.

Weiss, Y., Simoncelli, E. P., \& Adelson, E. H. (2002). Motion illusions as optimal percepts. Nature neuroscience, 5(6), 598-604.

Westheimer, G., \& Lavian, J. (2013). Perceptual learning of orientation judgments in oblique meridians. Attention, Perception, \& Psychophysics, 75(6), 1252-1259.

Wilson, H.R., Loffler, G., Wilkinson, F., \& Thistlethwaite, W.A. (2001). An inverse oblique effect in human vision. Vision Research, 41, 1749-1753.

Xing, J., \& Heeger, D. J. (2001). Measurement and modeling of center-surround suppression and enhancement. Vision research, 41(5), 571-583.

Yacoub, E., Harel, N., \& Uğurbil, K. (2008). High-field fMRI unveils orientation columns in humans. Proceedings of the National Academy of Sciences,105(30), 10607-10612.

Yang, B., Ma, X., Schweinhart, A.M., Wang, F., Sun, M., \& Song, Y. (2012). Comparison of event-related potentials elicited by cardinal and oblique orientations with broad-band noise stimuli. Vision Research. 60, 95-100.

Yu, C., Klein, S. A., \& Levi, D. M. (2002). Facilitation of contrast detection by crossoriented surround stimuli and its psychophysical mechanisms. Journal of Vision, 2(3), 4. 
Zhang, P., Bao, M., Kwon, M., He, S., \& Engel, S.A. (2009). Effects of orientationspecific visual deprivation induced with altered reality. Current Biology, 19, 1956-1960.

Ziemer, C. J., Plumert, J. M., Cremer, J., \& Kearney, J. (2007). Perceptual adaptation to environmental scale. Journal of Vision 7(9). 


\section{univensisty of LOUISVILLE}

Human Subjects Protection Program Office

MedCenter One - Suite 200

501 E. Broadway

Louisville, KY 40202-1798

Office: 502.852 .5188 Fax: 502.852 .2164
DATE:

TO:

FROM:

IRB NUMBER:

STUDY TITLE:

REFERENCE \#:

DATE OF REVIEW:

IRB STAFF CONTACT:
February 10, 2015

Edward A Essock

The University of Louisville Institutional Review Board

91.0272

Investigation of the Capabilities of the Human Visual System

342612

$02 / 09 / 2015$

Name: Jacqueline S. Powell

Phone: $852-4101$

Email: jspowe01@Louisville.edu

The continuation request for this study was reviewed by the Chair of the Institutional Review Board (IRB) through the expedited review procedure, according to 45 CFR 46.110 and 21 CFR 56.110 since this study falls under N/A Category 4: Collection of data through noninvasive procedures (not involving general anesthesia or sedation) routinely employed in clinical practice, excluding procedures involving $x$-rays or microwaves. Where medical devices are employed, they must be cleared/approved for marketing .

The study now has continued committee approval from 03/05/2015 through 03/04/2016.

The following items were reviewed and approved:

\begin{tabular}{|l|l|l|l|}
\hline \multicolumn{4}{|l|}{ Submission Components } \\
\hline Synopsis & Version 1.0 & $01 / 05 / 2015$ & Approved \\
\hline Protocol & Version 1.0 & $01 / 05 / 2015$ & Approved \\
\hline Informed Consent & Version 1.0 & $01 / 05 / 2014$ & Approved \\
\hline
\end{tabular}

Continuation Review Requirements

You are responsible for submitting a continuation review 30 days prior to the expiration date of your research study. Investigators who allow their study approval to expire have committed significant non-compliance with federal regulations. Such lapses may require reporting to federal agencies, a program audit by compliance auditors to ensure that subjects were not enrolled during the expired period, and may lead to findings of serious and continuing noncompliance if expiration were to occur a second time.

Please begin using your newly approved (stamped) consent on 3/5/2015.

This action will be reported promptly to the IRB at a scheduled full Board meeting. 
If you have any questions, please contact the HSPPO at (502) 852-5188 or hsppofc@louisville.edu

Thank you for your submission.

Sincerely,

Pate $m$ Lused

Peter M. Quesada, Ph.D., Chair

Social/Behavioral/Educational Institutional Review Board

PMQ/jsp 


\section{CURRICULUM VITAE}

April M. Schweinhart

Email: schweinharta@gmail.com

\section{Education}

University of Louisville

PhD

2015

Department of Psychological and Brain Sciences

Major Area: Experimental Psychology

Specialization: Vision and Hearing Sciences

University of Louisville

MS

2012

Department of Psychological and Brain Sciences

Major Area: Experimental Psychology

Specialization: Vision and Hearing Sciences

Hanover College

BA (cum laude)

2008

Department of Psychology

Department of Art

Major Area: Psychology and Studio Art

\section{Abstracts and Presentations}

Schweinhart, A.M. (2010, May). The Orientation Content of Art Images. Experimental Seminar Series. Lecture conducted from University of Louisville, Louisville, KY

Schweinhart, A. M., Essock, E. A., \& Kim, Y. (2011). Anisotropy in Paintings: A reflection of structural anisotropy in natural scenes? Journal of Vision, 11(11): 1163; doi:10.1167/11.11.1163. Abstract. Poster

Williams, H.G., Schweinhart, A.M., O’Keefe, E.M., Haun, A.M., \& Essock, E.A. (2012). Aesthetic Preference of Oriented Content: Relation to the Anisotropic Content of Natural Scenes. Journal of Vision, 12(9): 1084; doi:10.1167/12.9.1084. Abstract. Poster.

Schweinhart, A.M., Dubinchik, M., O’Keefe, E.M., Williams, H.G., \& Essock, E.A. (2012). Regularities in the Anisotropic Content of Portrait and Landscape Paintings: A Corollary to the Horizontal Effect Anisotropy of Visual Processing. Journal of Vision, 12(9); doi:10.1167/12.9.842. Abstract. Poster.

Schweinhart, A.M. \& Essock, E.A. (2012). Structural Regularities in Paintings: Correspondence to Natural Scenes and Human Visual Processing. Visual Science of Art Conference, Alghero, Italy. Abstract. Poster. 
Schweinhart, A., \& Essock, E. (2013). Painting What We See: Painters Over-Regularize Structure in the Environment. Journal of Vision, 13(9), 1240-1240. Abstract. Poster

Schweinhart, A.M. (2014, Nov.). Changing What You See Changes How You See: Analyzing the Plasticity of Broadband Orientation Perception Experimental Seminar Series. Lecture conducted from University of Louisville, Louisville, KY.

Schweinhart, A. M. (2015, Feb). Changing What You See Changes How You See: Analyzing the Plasticity of Broadband Orientation Perception Lecture conducted from University of Nevada, Reno, Reno, NV.

Schweinhart, A.M., Shafto, P., \& Essock, E.A., (2015). Effects of Recent Exposure to Atypical Environmental Statistics on orientation Perception: Analyzing the Plasticity of the Horizontal Effect. Vision Sciences Society Annual Meeting, St. Pete's Beach, FL. Abstract.Poster.

\section{Publications}

Yang, B., Ma, X., Schweinhart, A.M., Wang, F., Sun, M., \& Song, Y. (2012). Comparison of event-related potentials elicited by cardinal and oblique orientations with broad-band noise stimuli. Vision Research. 60, 95-100.

Schweinhart, A. M., \& Essock, E. A. (2013). Structural content in paintings: Artists overregularize oriented content of paintings relative to the typical natural scene bias. Perception, 42(12), 1311-1332.

Schweinhart, A.M. \& Essock, E.A. (submitted). Structural Content in Paintings II: Overregularization shown in paintings created from corresponding scenic referents.

Schweinhart, A.M., Shafto, P., \& Essock, E.A. (in prep). Adaptation alters priors to match just-viewed scenes optimizing orientation perception.

Schweinhart, A.M., Haun, A.M., \& Essock, E.A. (in prep). The relationship between anisotropic visual processing and aesthetic appeal.

\section{Research and Teaching Experience}

Spring 2015

May 2013-2014
Graduate Teaching Assistant - Sensation and Perception, Lifespan Development Department of Psychological and Brain Sciences, University of Louisville, Louisville, KY, USA

Guest Lecturer for PSYC 331: Sensation and Perception

"Sound and the Auditory System" (March 10 ${ }^{\text {th }}$ )

Guest Lecturer for PSYC 363: Lifespan Development

“Adolescence: Psychosocial Development" (March 31 ${ }^{\text {st }}$ )

"Emerging Adulthood: Body, Mind, \& Social World" (April 2 ${ }^{\text {nd }}$ )

"Adulthood: Body and Mind" (April 9 ${ }^{\text {th }}$ )

Graduate Teaching Assistant - Quantitative Methods for Psychology 
Weekly Lab Sessions

Department of Psychological and Brain Sciences, University of Louisville, Louisville, KY, USA

Fall 2013

Guest Lecturer for PSYC 509, Matlab

"Signal Processing" (Nov. $6^{\text {th }}$ )

Fall 2012

Guest Lecturer for PSYC 432, Seeing and Art

"Natural Scenes, Structure and Aesthetics" (Sep. 18 $8^{\text {th }}$ )

"Stereopsis and Depth in 2D Art" (Nov. 15 ${ }^{\text {th }}$ )

Aug 2011-May 2013 Graduate Research Assistant

Department of Psychological and Brain Sciences,

University of Louisville, Louisville, KY, USA

Jan 2010-Aug $2011 \quad$ Graduate Teaching Assistant - Introduction to Psychology and Cognitive Psychology

Department of Psychological and Brain Sciences,

University of Louisville, Louisville, KY, USA

\section{Grants, Funding, and Awards}

2010-2011 Graduate Research and Creative Activities grant

University of Louisville College of Arts and Sciences

2011-2012 Graduate Research Funds

University of Louisville College of Arts and Sciences Graduate Student Union

2011-2012 Graduate Student Travel Funds

University of Louisville College of Arts and Sciences Graduate Student Council

2013-2014 Graduate Student Research Funds

University of Louisville Graduate Student Council

2014-2015 Intramural Research and Creative Activities Grant

University of Louisville College of Arts and Sciences

Fall 2014 Dissertation Completion Award

University of Louisville School of Graduate and Interdisciplinary Studies 